\title{
Cochrane
}

Library

Cochrane Database of Systematic Reviews

\section{Mechanical versus manual chest compressions for cardiac arrest} (Review)

Wang PL, Brooks SC

Wang PL, Brooks SC.

Mechanical versus manual chest compressions for cardiac arrest.

Cochrane Database of Systematic Reviews 2018, Issue 8. Art. No.: CD007260.

DOI: 10.1002/14651858.CD007260.pub4.

www.cochranelibrary.com 
TABLE OF CONTENTS

HEADER 1

ABSTRACT

PLAIN LANGUAGE SUMMARY

SUMMARY OF FINDINGS

BACKGROUND

OBJECTIVES

METHODS

RESULTS

Figure 1.

Figure 2.

Figure 3.

DISCUSSION

AUTHORS' CONCLUSIONS

ACKNOWLEDGEMENTS

REFERENCES

CHARACTERISTICS OF STUDIES

DATA AND ANALYSES

Analysis 1.1. Comparison 1 Mechanical chest compressions versus manual chest compressions, Outcome 1 Survival to hospital discharge with good neurological function.

Analysis 1.2. Comparison 1 Mechanical chest compressions versus manual chest compressions, Outcome 2 Survival to hospital discharge.

Analysis 1.3. Comparison 1 Mechanical chest compressions versus manual chest compressions, Outcome 3 Return of spontaneous circulation.

Analysis 1.4. Comparison 1 Mechanical chest compressions versus manual chest compressions, Outcome 4 Survival to hospital admission.

Analysis 1.5. Comparison 1 Mechanical chest compressions versus manual chest compressions, Outcome 5 Sternal or rib fractures.

Analysis 1.6. Comparison 1 Mechanical chest compressions versus manual chest compressions, Outcome 6 Haemothorax or pneumothorax.

Analysis 1.7. Comparison 1 Mechanical chest compressions versus manual chest compressions, Outcome 7 Internal abdominal organ injury.

APPENDICES

WHAT'S NEW

HISTORY

CONTRIBUTIONS OF AUTHORS

DECLARATIONS OF INTEREST

SOURCES OF SUPPORT

INDEX TERMS 
[Intervention Review]

\section{Mechanical versus manual chest compressions for cardiac arrest}

Peter L Wang1,2, Steven C Brooks33,4

1Department of Medicine, Queen's University, Kingston, Canada. 2School of Medicine, Faculty of Health Sciences, Queen's University, Kingston, Canada. ${ }^{3}$ Department of Emergency Medicine, Queen's University, Kingston, Canada. ${ }^{4}$ Rescu, Li Ka Shing Knowledge Institute, Division of Emergency Medicine, Department of Medicine, University of Toronto, Toronto, Canada

Contact address: Steven C Brooks, Department of Emergency Medicine, Queen's University, Kingston, ON, Canada. brookss1@kgh.kari.net.

Editorial group: Cochrane Heart Group.

Publication status and date: New search for studies and content updated (no change to conclusions), published in Issue 8, 2018.

Citation: Wang PL, Brooks SC. Mechanical versus manual chest compressions for cardiac arrest. Cochrane Database of Systematic Reviews 2018, Issue 8. Art. No.: CD007260. DOI: 10.1002/14651858.CD007260.pub4.

Copyright @ 2018 The Cochrane Collaboration. Published by John Wiley \& Sons, Ltd.

\section{A B S T R A C T}

\section{Background}

Mechanical chest compression devices have been proposed to improve the effectiveness of cardiopulmonary resuscitation (CPR).

\section{Objectives}

To assess the effectiveness of resuscitation strategies using mechanical chest compressions versus resuscitation strategies using standard manual chest compressions with respect to neurologically intact survival in patients who suffer cardiac arrest.

\section{Search methods}

On 19 August 2017 we searched the Cochrane Central Register of Controlled Studies (CENTRAL), MEDLINE, Embase, Science Citation IndexExpanded (SCI-EXPANDED) and Conference Proceedings Citation Index-Science databases. Biotechnology and Bioengineering Abstracts and Science Citation abstracts had been searched up to November 2009 for prior versions of this review. We also searched two clinical trials registries for any ongoing trials not captured by our search of databases containing published works: Clinicaltrials.gov (August 2017) and the World Health Organization International Clinical Trials Registry Platform portal (January 2018). We applied no language restrictions. We contacted experts in the field of mechanical chest compression devices and manufacturers.

\section{Selection criteria}

We included randomised controlled trials (RCTs), cluster-RCTs and quasi-randomised studies comparing mechanical chest compressions versus manual chest compressions during CPR for patients with cardiac arrest.

\section{Data collection and analysis}

We used standard methodological procedures expected by Cochrane.

\section{Main results}

We included five new studies in this update. In total, we included 11 trials in the review, including data from 12,944 adult participants, who suffered either out-of-hospital cardiac arrest (OHCA) or in-hospital cardiac arrest (IHCA). We excluded studies explicitly including patients with cardiac arrest caused by trauma, drowning, hypothermia and toxic substances. These conditions are routinely excluded from cardiac arrest intervention studies because they have a different underlying pathophysiology, require a variety of interventions specific to the underlying condition and are known to have a prognosis different from that of cardiac arrest with no obvious cause. The exclusions were meant to reduce heterogeneity in the population while maintaining generalisability to most patients with sudden cardiac death. 
The overall quality of evidence for the outcomes of included studies was moderate to low due to considerable risk of bias. Three studies $(\mathrm{N}=7587$ ) reported on the designated primary outcome of survival to hospital discharge with good neurologic function (defined as a Cerebral Performance Category (CPC) score of one or two), which had moderate quality evidence. One study showed no difference with mechanical chest compressions (risk ratio (RR) 1.07, 95\% confidence interval ( $\mathrm{Cl}) 0.82$ to 1.39), one study demonstrated equivalence (RR $0.79,95 \% \mathrm{Cl} 0.60$ to 1.04 ), and one study demonstrated reduced survival (RR $0.41, \mathrm{Cl} 0.21$ to 0.79 ). Two other secondary outcomes, survival to hospital admission ( $N=7224$ ) and survival to hospital discharge $(\mathrm{N}=8067)$, also had moderate quality level of evidence. No studies reported a difference in survival to hospital admission. For survival to hospital discharge, two studies showed benefit, four studies showed no difference, and one study showed harm associated with mechanical compressions. No studies demonstrated a difference in adverse events or injury pattern setween comparison groups but the quality of data was low. Marked clinical and statistical heterogeneity between studies precluded any pooled estimates of effect.

\section{Authors' conclusions}

The evidence does not suggest that CPR protocols involving mechanical chest compression devices are superior to conventional therapy involving manual chest compressions only. We conclude on the balance of evidence that mechanical chest compression devices used by trained individuals are a reasonable alternative to manual chest compressions in settings where consistent, high-quality manual chest compressions are not possible or dangerous for the provider (eg, limited rescuers available, prolonged CPR, during hypothermic cardiac arrest, in a moving ambulance, in the angiography suite, during preparation for extracorporeal CPR [ECPR], etc.). Systems choosing to incorporate mechanical chest compression devices should be closely monitored because some data identified in this review suggested harm. Special attention should be paid to minimising time without compressions and delays to defibrillation during device deployment.

\section{PLAIN LANGUAGE SUMMARY}

\section{Mechanical chest compression machines for cardiac arrest}

\section{Review question}

We reviewed which method of chest compressions (applying the traditional hand technique versus using a machine) resulted in more lives saved during cardiopulmonary resuscitation (CPR) for cardiac arrest.

\section{Background}

'Sudden cardiac arrest' occurs when someone's heart stops beating unexpectedly. Cardiopulmonary resuscitation, referred to as CPR, involves rhythmical pushing on the chest of a cardiac arrest victim to provide forward blood flow. This can keep blood flowing to the victim's vital organs while the heart is not pumping. CPR has been shown to improve the chance that the heart will restart and the victim will survive. Machines have been developed to take over this chest pumping action using automated pistons, pneumatic vests, or bandlike mechanisms. The theory is that these machines should be able to provide a more effective pumping action than is seen in humans because the machines do not pause or get tired. Furthermore, they provide consistent pressure and timing of each chest compression in line with latest evidenced-based practice. Some preliminary studies using these machines have shown that they are easy to use and can save people with cardiac arrest. This is an update of the Cochrane Review on mechanical chest compression devices originally published in 2011 and updated last in 2014.

\section{Study characteristics}

The evidence is current to August 2017. We searched the literature and found a total of 2554 citations that were potentially relevant. After reviewing each of these, we found 11 articles describing clinical trials that could help us answer our question. Taken together, these trials included 12,944 adult participants who suffered cardiac arrest either in-hospital or out-of-hospital. The newest studies identified in this update are larger and of higher quality than those that had been identified in prior versions of this review. Several studies were sponsored by device manufacturers.

\section{Key results}

We found that available studies have important differences from one another. The most important differences were the type of mechanical device studied and the type of CPR protocol provided for patients assigned to the manual chest compression group. These differences make comparisons across studies challenging. Some studies reported improvements in rate of survival for patients treated with mechanical chest compressions compared to patients treated with manual chest compressions, while others reported no difference or even suggested harm associated with mechanical chest compressions. When considering all of the identified studies together, it seems like mechanical chest compression devices probably have a very similar effect on survival when compared with high-quality manual chest compressions.

\section{Quality of evidence}

With the inclusion of several large studies, the overall quality of evidence has improved considerably, and now may be considered to be of low to moderate quality. 
SUMMARY OF FINDINGS

\section{Summary of findings for the main comparison. Mechanical chest compressions compared to manual chest compressions for cardiac arrest}

\section{Mechanical chest compressions compared to manual chest compressions for cardiac arrest}

Patient or population: patients with cardiac arrest

Setting: in-hospital and out-of-hospital cardiac arrest

Intervention: mechanical chest compressions

Comparison: manual chest compressions

\begin{tabular}{|c|c|c|c|}
\hline Outcomes & Impact & $\begin{array}{l}\text { № of partici- } \\
\text { pants } \\
\text { (studies) }\end{array}$ & $\begin{array}{l}\text { Quality of } \\
\text { the evi- } \\
\text { dence } \\
\text { (GRADE) }\end{array}$ \\
\hline $\begin{array}{l}\text { Survival to hos- } \\
\text { pital discharge } \\
\text { with good neuro- } \\
\text { logical function }\end{array}$ & $\begin{array}{l}1 \text { study showed a decrease, } 1 \text { study showed no difference, and } 1 \text { study showed } \\
\text { equivalence in this outcome with mechanical devices. No meta-analysis was } \\
\text { possible due to heterogeneity. }\end{array}$ & $\begin{array}{l}7587 \\
(3 \mathrm{RCTs}) d\end{array}$ & $\begin{array}{l}\oplus \oplus \oplus \ominus \\
\text { Moderate } a\end{array}$ \\
\hline $\begin{array}{l}\text { Survival to hos- } \\
\text { pital discharge }\end{array}$ & $\begin{array}{l}1 \text { study showed a decrease, } 4 \text { studies showed no difference, and } 2 \text { studies } \\
\text { showed an increase in this outcome with mechanical devices. No meta-analysis } \\
\text { was possible due to heterogeneity. }\end{array}$ & $\begin{array}{l}8067 \\
(7 \mathrm{RCTs})\end{array}$ & $\begin{array}{l}\oplus \oplus \oplus \ominus \\
\text { Moderate } a\end{array}$ \\
\hline $\begin{array}{l}\text { Return of spon- } \\
\text { taneous circula- } \\
\text { tion }\end{array}$ & $\begin{array}{l}1 \text { study showed a decrease, } 4 \text { studies showed no difference, and } 3 \text { studies } \\
\text { showed an increase in this outcome with mechanical devices. No meta-analysis } \\
\text { was possible due to heterogeneity. }\end{array}$ & $\begin{array}{l}11,771 \\
\text { (8 RCTs) }\end{array}$ & $\begin{array}{l}\oplus \oplus \Theta \Theta \\
\text { Low a b }\end{array}$ \\
\hline $\begin{array}{l}\text { Survival to hos- } \\
\text { pital admission }\end{array}$ & $\begin{array}{l}\text { All } 4 \text { studies showed no difference in this outcome with mechanical devices. No } \\
\text { meta-analysis was possible due to heterogeneity. }\end{array}$ & $\begin{array}{l}7224 \\
\text { (4 RCTs) }\end{array}$ & $\begin{array}{l}\oplus \oplus \oplus \ominus \\
\text { Moderate } a\end{array}$ \\
\hline $\begin{array}{l}\text { Sternal or rib } \\
\text { fractures }\end{array}$ & $\begin{array}{l}6 \text { studies showed no difference, and } 1 \text { study showed an increase in this outcome } \\
\text { with mechanical devices. No meta-analysis was possible due to heterogeneity. }\end{array}$ & $\begin{array}{l}7469 \\
(7 \mathrm{RCTS})\end{array}$ & $\begin{array}{l}\oplus \oplus \Theta \Theta \\
\text { Low a c }\end{array}$ \\
\hline $\begin{array}{l}\text { Haemothorax or } \\
\text { pneumothorax }\end{array}$ & $\begin{array}{l}\text { All } 5 \text { studies showed no difference in this outcome with mechanical devices. No } \\
\text { meta-analysis was possible due to heterogeneity. }\end{array}$ & $\begin{array}{l}7316 \\
\text { (5 RCTs) }\end{array}$ & $\begin{array}{l}\oplus \oplus \Theta \ominus \\
\text { Low a c }\end{array}$ \\
\hline $\begin{array}{l}\text { Internal abdomi- } \\
\text { nal organ injury }\end{array}$ & $\begin{array}{l}\text { All } 5 \text { studies showed no difference in this outcome with mechanical devices. No } \\
\text { meta-analysis was possible due to heterogeneity. }\end{array}$ & $\begin{array}{l}7337 \\
(5 \mathrm{RCTs})\end{array}$ & $\begin{array}{l}\oplus \oplus \ominus \ominus \\
\text { Low a c }\end{array}$ \\
\hline
\end{tabular}

\section{GRADE Working Group grades of evidence}

High quality: We are very confident that the true effect lies close to that of the estimate of the effect

Moderate quality: We are moderately confident in the effect estimate: The true effect is likely to be close to the estimate of the effect, but there is a possibility that it is substantially different

Low quality: Our confidence in the effect estimate is limited: The true effect may be substantially different from the estimate of the effect

Very low quality: We have very little confidence in the effect estimate: The true effect is likely to be substantially different from the estimate of effect

$a$ Downgraded by one level for serious risk of bias in all included studies.

b Downgraded by one level for serious risk of inconsistency. Three studies showed benefit, one study showed harm, and four studies showed neither.

c Downgraded by one level for serious risk of imprecision. Most studies had wide $95 \%$ confidence intervals.

$d$ Randomized controlled trials 


\section{B A C K G R O U N D}

This is the second update of this review, originally published in 2011 (Brooks 2011), and subsequently updated in 2014 (Brooks 2014). It concluded that there was insufficient evidence from highquality, randomised controlled trials (RCTs) to determine whether mechanical chest compression devices are associated with benefit or harm in the treatment of cardiac arrest.

\section{Description of the condition}

Cardiac arrests, both occurring out-of-hospital (OHCA) and inhospital (IHCA), remain a major health problem in the USA and Canada (Heart and Stroke Foundation 2017; Mozaffarian 2016). Survival rates vary from approximately $5 \%$ to $50 \%$ depending on location (out-of-hospital versus in-hospital), geographic region and other characteristics of the event (Kleinman 2015). More than half of survivors of sudden cardiac arrest have brain damage of varying degrees (Herlitz 2000; Pusswald 2000; Young 2009). Many patients are comatose after cardiac arrest, with outcomes ranging from brain death to good recovery (Young 2009).

The goal of treatment for cardiac arrest patients is to achieve return of spontaneous circulation and favourable neurological function as early as possible while minimising end-organ damage and dysfunction. Cardiopulmonary resuscitation (CPR), which involves the delivery of rhythmical chest compressions, with or without ventilations, can sustain a minimal but crucial amount of blood flow to vital organs while attempts are made to restore spontaneous circulation.

\section{Description of the intervention}

Traditional CPR for cardiac arrest victims includes the delivery of rhythmical manual chest compressions by a human rescuer. An alternative technique is to deliver chest compressions with the use of a mechanical chest compression device. Several types of these are commercially available (Lurie 2002), and they employ a variety of compression mechanisms. Different mechanisms include loaddistributing bands, pistons and pneumatic vests. Load-distributing band-CPR involves a wide band of material attached to a short backboard, which is placed around the patient's thorax. The circumference of the band is mechanically and rhythmically shortened and lengthened. The change in circumference of the band simulates compressions delivered in standard manual CPR. Piston devices use compressed gas to drive a piston placed over the lower sternum of the patient. Some of these piston devices use a suction cup attachment to provide active compression/ decompression CPR. A pneumatic vest is similar to an oversized blood pressure cuff placed circumferentially around the patient's thorax (Halperin 1993). Chest compression is caused by rapid introduction of air into the vest. Several reports have demonstrated the feasibility of using mechanical chest compression devices in the prehospital setting (Ong 2006; Steen 2005).

\section{How the intervention might work}

Several investigations have demonstrated that early CPR is associated with improved survival in both adults and children (Hasselqvist-Ax 2017; Herlitz 1994; Naim 2017; Stiell 2004). For example, in a study of more than 30,000 OHCAs in Sweden between 1990 and 2011, CPR performed before the arrival of emergency medical services personnel was associated with a 30-day odds of survival that was more than twice as high as that associated with no CPR before emergency medical services arrival (adjusted odds ratio 2.15 , 95\% confidence interval (CI) 1.88 to 2.45 ; Hasselqvist-Ax 2017). In the Naim 2017 study, bystander CPR was independently associated with improved survival in a cohort of 3900 OHCAs in patients less than 18 (adjusted odds ratio $1.57,95 \% \mathrm{Cl} 1.25$ to 1.96).

The quality of chest compressions, as defined by the continuity, rate and depth of compression, may be associated with survival; these characteristics of CPR have been emphasised in the American Heart Association (AHA) guidelines for CPR and emergency cardiovascular care (Kleinman 2015). Several animal studies (Kern 2002; Yu 2002), and at least one human study (Eftestol 2002), have demonstrated an inverse relationship between chest compression interruption duration and short-term survival. Even short pauses in chest compressions for ventilations (four seconds) have resulted in a significant decline in the central haemodynamic pressures necessary for adequate cerebral and coronary perfusion (Ewy 2005). The importance of rate of compression has been demonstrated in several animal (Kern 1986; Maier 1984; Swart 1994), and human studies (Swenson 1988b), which show that higher rates of CPR (120 to 140 compressions/min) improve central haemodynamic measurements. Human observational studies have suggested that return of spontaneous circulation peaks at a compression rate of $125 / \mathrm{min}$ (Idris 2012), and that survival to hospital discharge is optimised with compressions between 100/ min to $120 /$ min (Idris 2015). Excessive rates may be detrimental to compression depth (Monsieurs 2012). Increased compression depth was related to increased incidence of return of spontaneous circulation in animal models of cardiac arrest (Babbs 1983; Bellamy 1984; Kern 1986). Stiell et al, in a large North American study, including 1029 OHCA participants, demonstrated an association between compression depth and improved return of spontaneous circulation, one-day survival and survival to hospital discharge (Stiell 2012).

Through the use of direct observation of actual cardiac arrests, recordings from automated external defibrillators (AEDs) and accelerometers, several studies have shown that chest compressions performed by trained professionals do not meet recommendations for compression rate, depth and continuity (Abella 2005a; Abella 2005b; Ko 2005; Wik 2005). For example, Wik and colleagues observed that chest compressions were halted for an average of $48 \%$ of the time during OHCAs (Wik 2005). While observing 67 IHCAs, Abella and colleagues observed that the chest compression rate was less than $90 / \mathrm{min}$ for $27 \%$ of the duration of the cardiac arrest, and compression depth was too shallow $37 \%$ of the time (Abella 2005a).

Rescuer fatigue has been identified as an important potential contributor to poor CPR quality (Hightower 1995; Ochoa 1998). Hightower and colleagues observed significant fatigue after only one minute of chest compressions on a mannequin. Correct chest compressions were performed $92 \%$ of the time during the first minute, $67.1 \%$ during the second minute and $39.2 \%$ during the third minute. By five minutes, only $18 \%$ of chest compressions were being performed correctly. Participants did not accurately identify the point of fatigue (Hightower 1995).

The use of mechanical chest compression devices has been proposed to provide high-quality chest compressions without the interruptions and fatigue associated with human-delivered chest compressions. These devices also liberate human rescuers from the duty of performing chest compressions, allowing them to 
perform other resuscitation tasks related to management and transportation of the cardiac arrest victim.

Some data from animal and human observational studies suggest that mechanical chest compressions may be superior to manual chest compressions in cardiac arrest. Animal studies comparing mechanical chest compressions versus manual chest compressions have shown that mechanical chest compressions produce improved cerebral, central and coronary blood flow (Halperin 2002; Halperin 2004; Rubertsson 2005; Timmerman 2003). Evidence also suggests improved survival in animal models of cardiac arrest (Ikeno 2006; Steen 2002). Several observational studies in humans have demonstrated improved outcomes with the use of mechanical chest compression devices (Casner 2005; Ong 2006; Steen 2005; Swanson 2005; Swanson 2006b). A recent meta-analysis of observational studies favoured mechanical compressions (odds ratio (OR) $1.42,95 \% \mathrm{Cl} 1.21$ to 1.67 ) for survival to hospital admission (Bonnes 2016).

\section{Why it is important to do this review}

Despite the enthusiasm generated by promising animal and observational human studies of mechanical chest compressions, clinical equipoise persists with regard to the true effectiveness of this therapy (Lewis 2006b). Most data on the effectiveness of mechanical chest compression devices derive from observational data. To date, six systematic reviews have been published (Bonnes 2016; Gates 2015; Li 2016; Ong 2006; Tang 2015; Westfall 2013). A meta-analysis of 12 randomised and observational studies investigating OHCA participants observed a positive association between the use of mechanical chest compression devices and the odds of return of spontaneous circulation (OR 1.53, 95\% Cl 1.32 to 1.78; Westfall 2013). The authors of this review were employees of or had received a significant amount of research funding from Zoll Medical Corporation. Zoll Medical Corporation makes the AutoPulse mechanical CPR device. A systematic review including non-randomised studies showed no improvement in survival and the potential for worse neurological outcomes with mechanical devices (Ong 2006). A recent meta-analysis of nine prospective studies (both randomised and observational) of OHCAs and IHCAs reported that there was no difference in survival to discharge with good neurological function (risk ratio (RR) $1.11,95 \% \mathrm{Cl} 0.95$ to 1.3 ), but that return of spontaneous circulation was more likely to be achieved with manual compressions in OHCA (RR 0.87, 95\% Cl 0.81 to 0.94 ) and IHCA (RR $0.71,95 \% \mathrm{Cl} 0.53$ to 0.97 ) (Li 2016). Three recent systematic reviews performed a meta-analysis of the same five RCTs for OHCAs published between 2006 and 2015 (Bonnes 2016; Gates 2015; Tang 2015). These studies found no difference in survival outcomes, including survival to discharge (Gates: OR $0.89,95 \% \mathrm{Cl} 0.77$ to 1.02 ), survival to hospital admission (Bonnes: OR $0.94,95 \% \mathrm{Cl} 0.84$ to 1.05 ) and survival to discharge with good neurological outcome (Tang: RR $0.80,95 \% \mathrm{Cl} 0.61$ to 1.04). One of the reviews (Tang 2015), found a weak association between mechanical compressions and survival to hospital admission (RR of death $0.94,95 \% \mathrm{Cl} 0.89$ to 1.00 ) and to hospital discharge (RR of death $0.88,95 \% \mathrm{Cl} 0.78$ to 0.99 ). The latest 2015 guidelines from the International Liaison Committee on Resuscitation (ILCOR) Advanced Life Support (ALS) Task Force has recommended against the routine use of mechanical chest compressions. However, the authors do suggest that mechanical compressions may be an option in scenarios where sustained high-quality compressions would be otherwise impractical or unsafe (Callaway 2015).
For this 2017 update, the review authors have opted to forgo a quantitative meta-analysis, given the considerable clinical and methodological heterogeneity of the studies identified in the literature. Instead, this update serves to be a single unified review of the existing evidence from the 11 RCTs identified in the literature.

\section{O B J E C T IVES}

To assess the effectiveness of mechanical chest compressions versus standard manual chest compressions with respect to neurologically intact survival in patients who suffer cardiac arrest.

\section{METHODS}

\section{Criteria for considering studies for this review \\ Types of studies}

All studies employing patient-level randomisation or cluster randomisation comparing compressions delivered via any type of powered mechanical chest compression device versus standard manual chest compressions were considered for inclusion in the review. We also included studies that were quasi-randomised, which are controlled clinical trials in which the method of allocation is known but is not considered strictly random. Examples of quasi-random processes for assigning treatments include oddeven numbers, participant social security numbers, days of the week, participant record numbers, ambulance run numbers and participant birth dates, which may appear to represent 'random' phenomena but do not constitute a truly random process of determining group allocation. For a study to be included in the review as a quasi-randomised study, the methodology had to explicitly use the term "quasi-randomisation" to describe the method of allocation. We did not include prospective studies without random or quasi-random allocation in the review. We excluded studies with designs that involved cross-over of individual participants from the manual chest compression arm to the mechanical chest compression arm.

\section{Types of participants}

We considered for inclusion in the review patients suffering out-ofhospital cardiac arrest (OHCA) or in-hospital cardiac arrest (IHCA), with resuscitation attempted by trained medical personnel. We excluded studies explicitly including patients with cardiac arrest caused by trauma, drowning, hypothermia and toxic substances. These conditions are routinely excluded from cardiac arrest intervention studies because they have a different underlying pathophysiology, require a variety of interventions specific to the underlying condition and are known to have a prognosis different from that of cardiac arrest with no obvious cause. The exclusions were meant to reduce heterogeneity in the population while maintaining generalisability to most patients with sudden cardiac death.

\section{Types of interventions}

We considered for inclusion studies comparing compressions delivered via any type of powered, automatic mechanical chest compression device versus standard manual chest compressions provided by a human. 


\section{Types of outcome measures}

\section{Primary outcomes}

The primary outcome for this review was survival to hospital discharge with good neurological function, equivalent to a Cerebral Performance Category (CPC) one or two (Jennett 1975), as measured by any validated scale.

\section{Secondary outcomes}

Secondary survival outcomes included:

- survival to hospital discharge

- return of spontaneous circulation

- survival to emergency department arrival or hospital admission (OHCA only)

- short-term survival (less than or equal to 30 days)

- long-term survival (greater than 30 days)

- adverse effects

* sternal or rib fractures

* haemothorax or pneumothorax

* abdominal organ injury

We also sought to abstract time intervals that may negatively impact outcome, including:

- Emergency telephone call or scene arrival to first shock interval for ventricular fibrillation/ventricular tachycardia

- Emergency telephone call or scene arrival to first CPR interval

- Emergency telephone call or scene arrival to first return of spontaneous circulation interval

\section{Search methods for identification of studies}

\section{Electronic searches}

We searched the following electronic databases.

- Cochrane Central Register of Controlled Studies (CENTRAL; 2017, Issue 8) in the Cochrane Library (searched 19 August 2017).

- MEDLINE (Ovid, 1946 to August 2017, week 3) on 19 August 2017

- Embase (Ovid, 1980 to 18 August 2017, week 33) on 19 August 2017

- Science Citation Index-Expanded (SCI-EXPANDED) on the Web of Science (Thomson Reuters, 1970 to 19 August 2017) on 19 August 2017

- Conference Proceedings Citation Index-Science (CPCI-S) on the Web of Science (1990 to 19 August 2017) on 19 August 2017

- Science Citation abstracts on the Web of Science (Thomson Reuters, 1960 to 18 November 2009) on 18 November 2009

- Biotechnology and bioengineering abstracts (1982 to 18 November 2009) on 18 November 2009.

The search strategies used for each database are listed in Appendix 1 (for 2017), Appendix 2 (for 2014) and Appendix 3 (for 2011). The original review search strategy for MEDLINE includes the highly sensitive search filter for retrieval of reports of controlled trials (Higgins 2006). The updated MEDLINE and Embase searches include an updated RCT filter. For MEDLINE, we used the sensitivitymaximising version of the Cochrane RCT filter (Lefebvre 2011), and applied adaptations of it to Embase, SCI-EXPANDED and CPCI-S .
We imposed no language of publication restrictions. We sought translations through Cochrane for full-text articles in languages other than English.

\section{Searching other resources}

We searched the ClinicalTrials.gov clinical trials registry on 19 August 2017 (www.clinicaltrials.gov), and the World Health Organization International Clinical Trials Registry Platform on 17 January 2018 (www.who.int/ictrp/en). We handsearched bibliographies of included papers. We contacted an expert in the field of mechanical chest compression devices (Dr. James Christensen, University of British Columbia, 19 June 2007) and representatives of chest compression device manufacturers (Zoll, Medtronic, 19 June 2007) about published and unpublished studies on this topic for the original review. We accomplished contact with experts for subsequent searches (2010 and 2017) through author (SCB and LJM) participation in the International Liaison Committee on Resuscitation Advanced Life Support Task Force, which included international experts in OHCA and the use of mechanical chest compression devices. This committee of experts undertook a comprehensive review of the world literature on mechanical chest compression devices.

\section{Data collection and analysis}

\section{Selection of studies}

Review authors used predefined inclusion criteria to decide on the status of each citation. Two review authors (SCB and BLB in 2011, SCB and NH in 2014, SCB and PW in 2017) screened citations in an independent, hierarchical fashion by title, abstract and then full article for relevance. At each stage of review, we classified citations as 'include', 'exclude' or 'indeterminate'. This process was summarized using a PRISMA flowchart (Moher 2009). We included citations classified as 'include' or 'indeterminate' by at least one of the review authors in the next level of review. We planned to resolve disagreements at the full article stage through consensus and with the assistance of a third reviewer who was an author on prior versions of the review (LJM) if consensus could not be reached. A third review author was not required at any time for the 2017 review. Agreement between review authors at each stage of review was quantified using a kappa statistic. We recorded and reported the reason for exclusion at the full article stage in the results section of the review (Excluded studies).

\section{Data extraction and management}

Two review authors (SCB and LJM in 2011, SCB and NH in 2014, SCB and PW in 2017) abstracted data independently using a preformed data abstraction tool developed for the original review. We resolved discrepancies in data abstraction through consensus, but if we could not reach consensus, we planned to involve a third reviewer who was an author on prior updates for this review (BLB) to resolve discrepancies. When available, data abstraction included:

\section{Methods}

- year of study

- study design

- study setting

\section{Participants}

- sample size 
- number of participants per treatment arm

- mean age

- gender

- initial rhythm

- hypothermia treatment post-arrest

- inclusion criteria

- exclusion criteria

\section{Interventions}

- intervention

- comparison

- mechanical device employed

- detail of resuscitation protocol

\section{Outcomes}

- predefined primary and secondary outcomes

\section{Notes}

- notable differences from currently accepted resuscitation guidelines

- comments on unique aspects of study

\section{Assessment of risk of bias in included studies}

In the original review and 2014 update, one review author (SCB) evaluated each included study for risk of bias as recommended in the Cochrane Handbook for Systematic Reviews of Interventions (Higgins 2011). In this 2017 update, two review authors (SCB and PW) independently reviewed the risk of bias for all studies. This included making judgements on the following domains as having a low, unclear or high risk of bias: (1) random sequence generation, (2) allocation concealment, (3) incomplete outcome data, (4) cointervention or contamination, (5) blinding of care providers, (6) blinding of outcome assessors, and (7) other potential sources.

\section{Measures of treatment effect}

We quantified the primary outcome of survival to hospital discharge with good neurological function and dichotomous secondary outcomes, including survival at different time points and adverse events, as a risk ratio (RR) with $95 \%$ confidence intervals (Cls). We planned to compare continuous secondary outcomes (time interval data) by comparing differences in means.

\section{Unit of analysis issues}

For cluster-randomised trials, we planned to adjust for clustering by calculating effective sample sizes. We contacted the authors of the Hallstrom study, and determined a mean cluster size for the 223 clusters having at least one primary case was 3.44 (standard deviation 4.82; Hallstrom 2006). The intra-cluster correlation (ICC) was -0.005 . For the PARAMEDIC study, the ICC was reported to be negligible at 0.001 (Perkins 2015). Because the calculated design effects for both studies were so close to unity, we entered the data into Review Manager 5 for RR calculation without adjustment for clustering (Higgins 2011; Review Manager 2014). For trials with multiple comparator groups (e.g. different mechanical devices) we combined data from all mechanical device groups into one mechanical chest compression comparator group.

\section{Dealing with missing data}

We had planned to contact the study authors directly for any missing or ambiguous data, and to allow for a one-month period for response. If no response, this would be reported as missing data. However, we did not encounter missing data that required us to do so.

\section{Assessment of heterogeneity}

We qualitatively explored the clinical heterogeneity of included trials through a detailed examination of study characteristics. We used the $I^{2}$ statistic to quantify statistical heterogeneity and to determine the appropriateness of pooling results across studies. We planned a priori not to pool studies if $\mathrm{I}^{2}$ was $>50 \%$, indicating substantial statistical heterogeneity.

\section{Assessment of reporting biases}

We had planned to explore the data graphically with a funnel plot to look for evidence of publication bias, but we found too few studies for this to be useful.

\section{Data synthesis}

In this 2017 update, in line with changes in the MECIR standards, we explicitly evaluated the quality of evidence for each outcome using the GRADE approach (Ryan 2016). Given that all outcomes were from RCTs, the starting rating of evidence was 'high quality'. We downgraded quality by one level for serious concerns and two levels for very serious concerns regarding risk of bias, inconsistency, indirectness, imprecision, and publication bias. Two review authors (SCB and PW) independently rated the evidence. We used the GRADE profiler software to generate a 'Summary of findings' table for the following outcomes (GRADEpro GDT 2015).

- Survival to hospital discharge with good neurological function

- Survival to hospital discharge

- Return of spontaneous circulation

- Survival to hospital admission

- Sternal or rib fractures

- Haemothorax or pneumothorax

- Internal abdominal organ injury

We planned to use the DerSimonian and Laird method (randomeffects model) to provide a pooled estimate for RR when the data allowed. We planned to use a random-effects model to provide a conservative estimate of pooled effect and anticipated clinical heterogeneity amongst included studies. We made the decision not to pool study results given substantial clinical and statistical heterogeneity.

\section{Subgroup analysis and investigation of heterogeneity}

Planned a priori subgroup analyses included analysis by device type (piston versus load-distributing bands versus other), first rhythm analysed (ventricular fibrillation/ventricular tachycardia versus asystole/pulseless electrical activity/non-shockable) and location of arrest (IHCA versus OHCA). We made the decision to not perform pooled subgroup analysis because of issues with heterogeneity and insufficient data. 


\section{Sensitivity analysis}

We planned for sensitivity analyses including removal of data assessed to be at moderate or high risk of bias during quality review and removal of studies with cluster randomisation. We made the decision to not perform a sensitivity analysis due to heterogeneity preventing meta-analysis and a paucity of quality data in each category.

\section{RES U L T S}

\section{Description of studies}

\section{Results of the search}

A comprehensive updated search of the literature in 2017 identified 599 additional citations. In the original review, the comprehensive search identified 1561 citations (MEDLINE 516, Embase 517, Science Citation abstracts 275, Biotechnology and bioengineering abstracts 18, Cochrane CENTRAL 78, ClinicalTrials.gov 33, handsearches of references of included papers 7, contact with industry representatives and experts 117). The updated search conducted for the 2014 update identified an additional 390 citations (MEDLINE 40, Embase 50, Conference Proceedings Citation Index-Science and Science Citation Index-Expanded on Web of Science 186, Cochrane CENTRAL 34, ClinicalTrials.gov 80). The present update searched in 2017 further identified 599 citations (MEDLINE 43, Embase 143, Conference Proceedings Citation Index-Science and Science Citation Index-Expanded on Web of Science 258, Cochrane CENTRAL 48, ClinicalTrials.gov 24, World Health Organization (WHO) International Clinical Trials Registry 87). Two independent review authors (SCB and BLB in 2011, SCB and NH in 2014, SCB and PW in 2017) reviewed 2554 citations by title, and categorised 279 of these as 'include or 'indeterminate' and selected them for review by abstract. After review by abstract, we identified 68 citations as potentially relevant and reviewed the full-text. Eleven studies met all inclusion criteria and we included them in this review (Characteristics of included studies; Figure 1). 
Figure 1. PRISMA Study flow diagram.

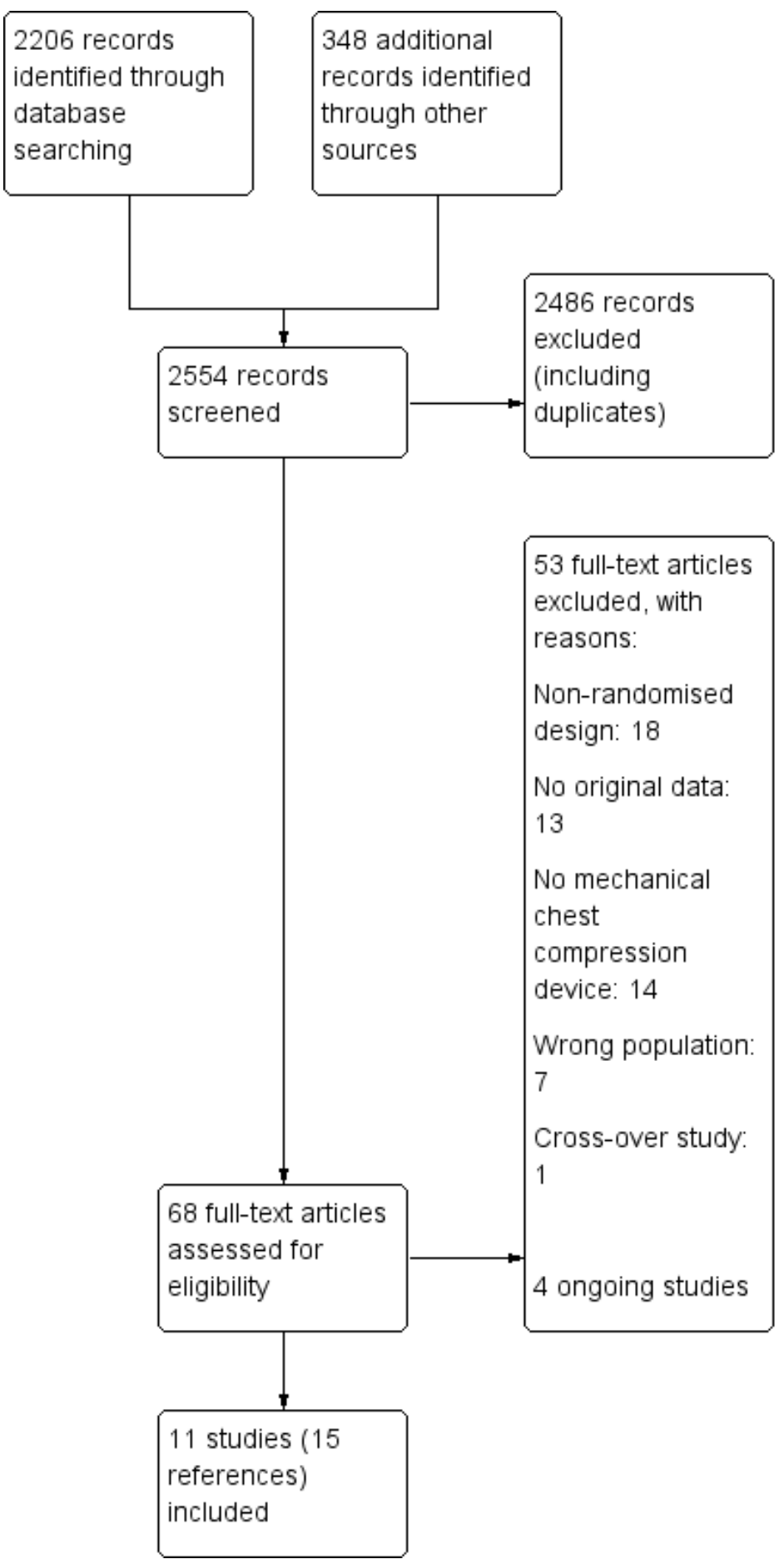

Agreement for relevance between review authors was evaluated by the kappa statistic for this update. Fair agreement was seen for titles and abstracts (0.87) and perfect agreement for full articles (1.0).

Three studies previously identified as ongoing in the 2014 update have now completed and published their results (Perkins 2015; Rubertsson 2014; Wik 2014). We identified four ongoing studies through a search of the WHO International Clinical
Trials Registry Platform (CTRI/2013/07/003840; ISRCTN38139840; ISRCTN78354073; NCT01521208; Characteristics of ongoing studies).

\section{Included studies}

In total, we included 11 studies in this review (12,944 participants). Of the five new studies included in the 2017 update, three are largescale randomised controlled trials (RCTs) which account for $90 \%$ of all participants included in the review (Perkins 2015; Rubertsson 
2014; Wik 2014). Reporting of participant characteristics was incomplete in several of the included studies. Reported mean ages of included participants ranged from 45.5 to 71 years old and the proportion who were males ranged from $58 \%$ to $68 \%$. The proportion of participants with initial shockable rhythms (ventricular fibrillation/ventricular tachycardia) varied between studies from $13 \%$ to $47 \%$.

While the ethnicity of the participants was not reported, three studies were conducted solely in the USA (Dickinson 1998; Halperin 1993; Taylor 1978), two studies were from China (Gao 2016; Lu 2010), one study was from Sweden (Smekal 2011), one study was from the Netherlands (Koster 2017), and one study was from the UK (Perkins 2015). The remaining studies were multinational collaborations between Sweden/Netherlands/ UK (Rubertsson 2014), USA/Austria/Netherlands (Wik 2014), and USA/Canada (Hallstrom 2006).

The use of mechanical cardiopulmonary resuscitation (CPR) for in-hospital cardiac arrest (IHCA) has been studied in four small-scale RCTs (sample size ranged from 34 to 218) with 452 participants total (Halperin 1993; Lu 2010; Taylor 1978; Koster 2017). There is substantial heterogeneity in the methodology, intervention algorithm used and reporting of results. Devices used to deliver mechanical CPR differed in all three studies, including an unspecified piston device (Taylor 1978), the Thumper device (a gaspowered external chest compressor) (Lu 2010), a pneumatic vesttype device (developed by the study authors) (Halperin 1993), and both the LUCAS and AutoPulse devices (Koster 2017).

Out-of-hospital cardiac arrest (OHCA) has been studied in eight RCTs with 12,492 participants (Dickinson 1998; Gao 2016; Hallstrom 2006; Koster 2017; Perkins 2015; Rubertsson 2014; Smekal 2011; Wik 2014). The sample size and quality of the data has improved considerably with the addition of the five recent RCTs newly included in this update.

Of the eight studies, one quasi-randomised trial evaluated the Thumper device (Dickinson 1998), four RCTs evaluated the AutoPulse device (Gao 2016; Hallstrom 2006; Koster 2017; Wik 2014), and three RCTs (Koster 2017; Rubertsson 2014; Smekal 2011), and one cluster-randomised trial (Perkins 2015), evaluated the LUCAS device. The AutoPulse is an automated, battery-powered external chest compressor that uses a load-distributing band to rhythmically compress the chest against a backboard. The LUCAS device employs active compression-decompression of the chest wall by using a silicone rubber cup (which creates a suction effect) and a pneumatic cylinder connected to a stiff back-plate.

In a multicentre, cluster-randomised trial, Hallstrom and colleagues studied 767 participants with OHCA (Hallstrom 2006). The primary outcome of this study was survival to four hours after the emergency call. The trial was stopped at interim analysis by the data safety and monitoring board because of decreased survival to hospital discharge with good neurological function among participants who received mechanical chest compressions.

The CIRC (Circulation Improving Resuscitation Care) trial, using the AutoPulse, was conducted by Wik and colleagues (Wik 2014), and it attempted to address some of the concerns in the Hallstrom
2006 study. Here, they looked at the outcomes of OHCA in 4231 adults. Patients randomised to the intervention arm received what the authors called 'integrated-AutoPulse CPR (iA-CPR)', which was intended to minimise hands-off time by using assigned responder roles and a standardised deployment strategy. This study is also noted for its effort to track and ensure high-quality CPR delivery in both arms of the trial through vigorous training and CPR quality monitoring. The primary outcome in the CIRC trial was survival to hospital discharge.

In a study by Smekal and colleagues (Smekal 2011), 148 cardiac arrest participants were randomly assigned to receive mechanical CPR performed with the LUCAS device or CPR performed with manual chest compressions. Recently, these authors have followed up and reported their results from their much larger LINC trial (LUCAS in Cardiac Arrest) involving 2589 adult OHCAs (Rubertsson 2014). The primary outcome was four-hour survival.

The PARAMEDIC trial investigated the LUCAS device and involved 4471 participants (Perkins 2015). They employed a clusterrandomised open-label controlled trial to compare adult 30day survival after OHCA between standing manual compressions and using the LUCAS-2 mechanical device. The study authors have emphasised the pragmatic nature of this trial. The primary outcome was 30 -day survival.

There is also a smaller, single-centre cluster-RCT $(\mathrm{N}=133)$ that was localised to the northern district of Shanghai, China which used the AutoPulse device (Gao 2016). Ten ambulances from one emergency medical centre were randomised to be equipped with the device or not and then were dispatched in consecutive order (ambulances 1 through 10) for suspected OHCA. The primary outcome for this study was return of spontaneous circulation.

The Koster 2017 study included 374 participants (156 with IHCA and 218 with OHCA) who were randomly assigned to either the AutoPulse, LUCAS or manual compressions. This was a singlecentre randomised controlled non-inferiority trial comparing the use of each device against protocols employing manual chest compressions only. This was a study designed to assess safety of the mechanical devices, with the primary outcome being "serious or life-threatening visceral resuscitation-related injury". The devices were not directly compared in this study.

\section{Excluded studies}

Common reasons for exclusion were that the article described an experiment involving the use of a non-powered active compression-decompression device as an adjunct to manual CPR (such as a manually operated suction cup) and that the article described a narrative literature review and provided no original data (Excluded studies).

\section{Risk of bias in included studies}

The results of our assessment for risk of bias in included studies can be seen in the Characteristics of included studies table and are summarised in Figure 2 and Figure 3. The overall quality of the available data has improved considerably with the three large-scale RCTs included in this update (Perkins 2015; Rubertsson 2014; Wik 2014), along with two smaller studies (Gao 2016; Koster 2017). 
Figure 2. Risk of bias graph: review authors' judgements about each risk of bias item presented as percentages across all included studies.

Random sequence generation (selection bias)

Allocation concealment (selection bias)

Blinding of participants and personnel (performance bias)

Blinding of outcome assessment (detection bias)

Incomplete outcome data (attrition bias)

Selective reporting (reporting bias)

Other bias

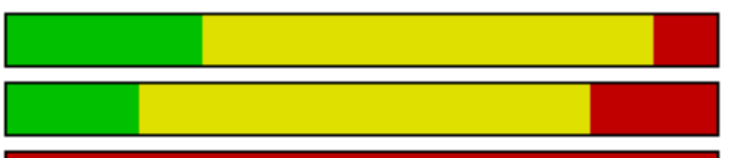

L

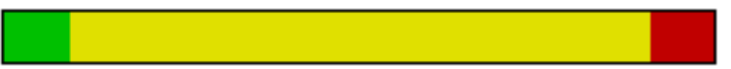

L

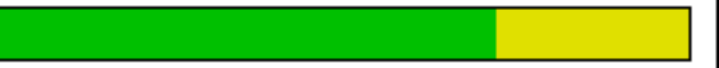

L

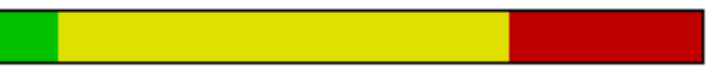

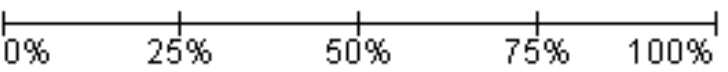

Low risk of bias

Unclear risk of bias

High risk of bias 
Figure 3. Risk of bias summary: review authors' judgements about each risk of bias item for each included study.

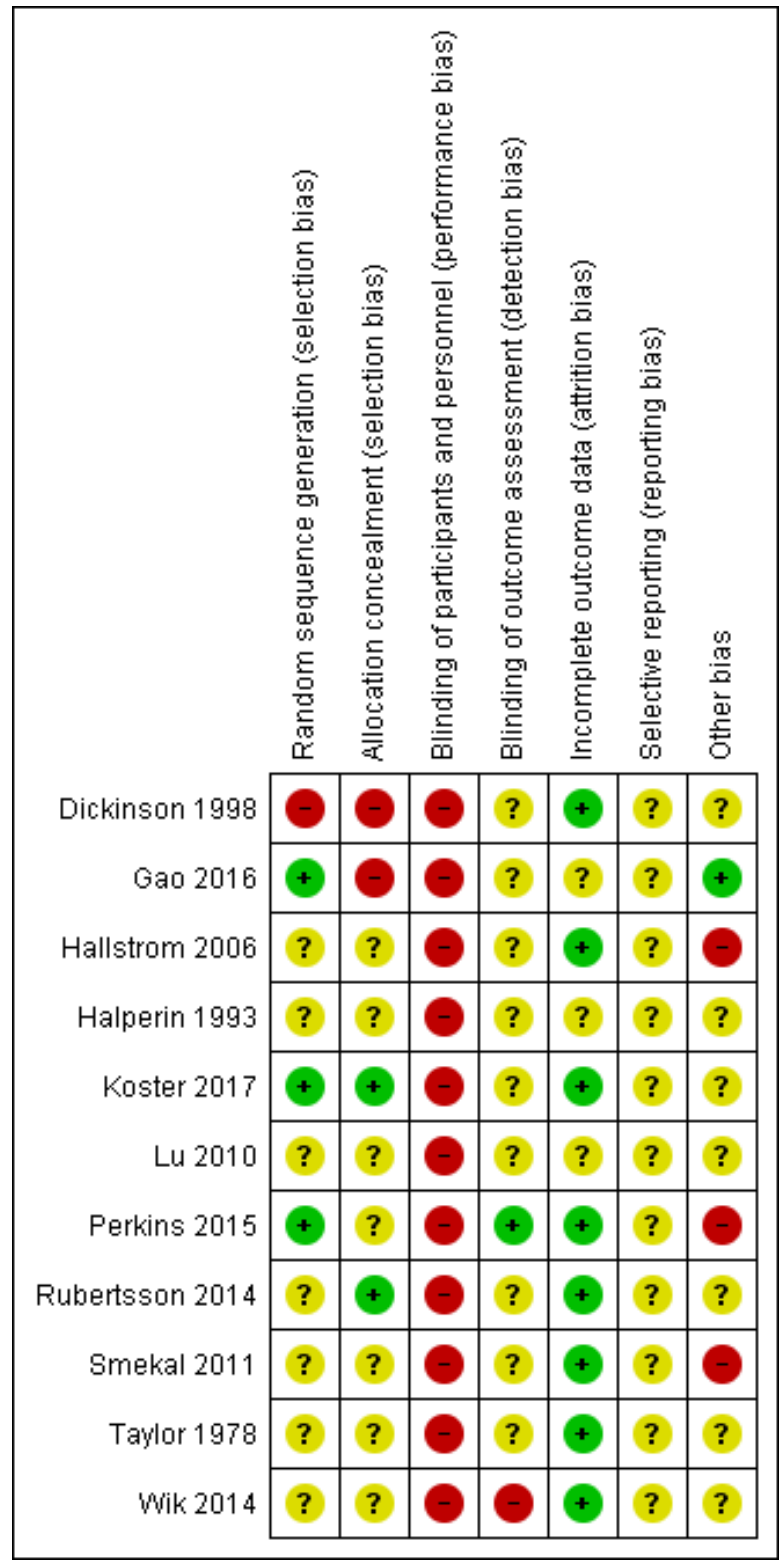

\section{Allocation}

\section{Random sequence generation}

Three of the newly included studies, using a cluster randomisation study design, described using a computer-generated sequence to randomise ambulance vehicles, placing them at low risk of bias with respect to the randomisation process (Gao 2016; Koster 2017; Perkins 2015). In four studies using simple randomisation (Halperin 1993; Lu 2010; Smekal 2011; Taylor 1978), and one study using cluster randomisation (Hallstrom 2006), the method of random allocation was not adequately reported. Two other studies referred to the use of randomisation envelopes, although they did not report on the specific method of generating the sequence (Rubertsson 2014; Wik 2014). We judged these seven studies to also have an unclear risk of bias. One study was self-described as "quasirandomised" using odd and even days (Dickinson 1998), placing it at a high risk of bias.

\section{Allocation concealment}

Two studies adequately described their method of allocation concealment, including measures to protect against foreknowledge and track attempts to subvert the randomisation by using opaque, sealed envelopes (Koster 2017; Rubertsson 2014). If the use of opaque envelopes was not mentioned explicitly, we deemed the risk of bias to be unclear (Hallstrom 2006; Lu 2010; Perkins 2015; Taylor 1978). For two studies, we felt that the risk of bias was high (Dickinson 1998; Gao 2016). For example, in the Gao cluster-randomised trial, ambulances were randomised to be carrying the mechanical chest compression device or not. Ambulances were dispatched to OHCA emergencies, but it is not clear whether dispatchers might send ambulances carrying the mechanical device to suspected OHCA event with particular characteristics preferentially. Some of the baseline characteristics between treatment groups are different (e.g. initial cardiac arrest rhythm and witness status), suggesting this source of selection 
bias might have occurred. The Dickinson 1998 study was quasirandomised using odd and even days. For studies that did not mention allocation concealment, the risk of bias was unclear (Halperin 1993; Smekal 2011; Wik 2014).

\section{Blinding}

In all studies, since the blinding of study personnel was not feasible given the nature of the intervention, we judged these to be at a high risk of performance bias. The CIRC trial mentions that study personnel were not always blinded to the study arm, although the extent and reason were not explained, thus placing it at a high risk of detection bias (Wik 2014). Blinding of outcome assessors was only addressed by the PARAMEDIC study (blinding of research nurses) and the study by Koster et al (blinding of study assessors) (Koster 2017; Perkins 2015). We thus judged the PARAMEDIC study to be at low risk of detection bias (Perkins 2015). However, we determined that the risk of bias for the Koster study was unclear as it mentions that the pathologists performing the autopsies could not be completely blinded due to occasional skin markings on the chest left by mechanical devices (Koster 2017).

\section{Incomplete outcome data}

Most trials had excellent follow-up and very few withdrawals for most outcomes, placing them at a low risk of bias (Dickinson 1998; Hallstrom 2006; Koster 2017; Perkins 2015; Rubertsson 2014; Smekal 2011; Taylor 1978; Wik 2014). One study did not report the number of drop outs and as such, the risk was unclear (Halperin 1993). With respect to neurologic outcomes, the CIRC trial notes that this was not known 26\% (manual compression) and 30\% (mechanical compression) of survivors. However it is unlikely to be a significant source of bias given that the loss is similar in both arms (Wik 2014). Two studies did not explicitly report incomplete or loss to follow-up data, thus the risk is unclear (Gao 2016; Lu 2010).

\section{Selective reporting}

Selective reporting bias was difficult to determine because the protocols of several included studies were not available. Two studies chose to report survival to four hours as the primary outcome (Hallstrom 2006; Rubertsson 2014), which is uncommon and not recognised by international consensus groups as a key standard outcome (Utstein 2015). However, all but the Taylor 1978 study and the PARAMEDIC trial (Perkins 2015), reported all outcomes stated in the methods. The PARAMEDIC study did not report on survival to hospital discharge as originally intended in the study protocol because they felt that survival to 30 days was more clinically relevant (Perkins 2010a), and they were unable to completely collect data from hospitals (Perkins 2015).

Seven trials reported adverse effects such as rib or sternal fracture, haemothorax or pneumothorax or other internal abdominal organ injury (Gao 2016; Halperin 1993; Koster 2017; Lu 2010; Rubertsson 2014; Taylor 1978; Wik 2014). Three studies did so with the use of autopsy data, which were carried out on a minority of participants (Halperin 1993; Koster 2017; Taylor 1978). Two studies also did not specify the criteria for autopsy (Halperin 1993; Taylor 1978).The LINC and CIRC trials relied on reports of adverse events by emergency and hospital personnel (Rubertsson 2014; Wik 2014). There is the potential for selective investigation, or reporting of adverse events, or both, which may incorporate significant potential bias into these data.

\section{Other potential sources of bias}

\section{Cointervention or contamination}

Cointervention or contamination were present in several studies (for studies that were already included in the previous review, see Risk of bias in included studies). Notably, the PARAMEDIC study had unbalanced cross-over (39\% mechanical to manual; < $1 \%$ manual to mechanical) that may understate the effect of mechanical CPR, if effective (Perkins 2015). This was determined to be at a high risk of bias. The LINC study also used an unconventional CPR algorithm where the mechanical CPR group received a defibrillator shock without pulse/rhythm check and followed three-minute CPR periods (Rubertsson 2014). It is unclear what the impact this cointervention may have had on the measured outcomes.

\section{Differences at baseline and potential confounders}

There was inadequate reporting, or significant differences between the study arms with respect to baseline characteristics, or both, that may potentially act as important confounders. Four of the 11 studies failed to report baseline participant characteristics, including the suspected cause of the cardiac arrest, the circumstances of the cardiac arrest (e.g. witnessed versus unwitnessed), the initial cardiac arrest rhythm and other important prognostic factors (Dickinson 1998; Halperin 1993; Lu 2010; Taylor 1978). The impact of these potential confounding variables was impossible to assess, which raises the possibility of selection and allocation bias, and was deemed to be an unclear risk of bias. In general, reporting of the randomisation process was inadequate.

In the Hallstrom 2006 study, the distribution of body habitus was dissimilar between groups. More "thin" and "morbidly obese" participants were included in the mechanical chest compression group. The impact of this imbalance was not explored in the analysis. Intuitively, body shape may interact with treatment effect because of the physics of the mechanical device. Also, among participants with ventricular fibrillation or pulseless ventricular tachycardia, those in the mechanical CPR group had an additional 2.1-minute delay from the emergency call to first defibrillation attempt compared with those in the manual CPR group. This important difference in a well-recognised prognostic factor for survival was not further explored as a potential modifier of effect. Taken together, this study is at a high risk of bias. Two studies had a markedly higher occurrence of ventricular fibrillation or pulseless ventricular tachycardia as the initial rhythm in the mechanical group compared to the manual group $(47.1 \%$ versus $17 \%$ in the Halperin study; and $24 \%$ versus $21 \%$ in the CIRC study). This was not addressed by Halperin in the text or analysis of the study (Halperin 1993), thus at an unclear risk of bias. In many cases, reporting of baseline characteristics and known prognostic factors for cardiac arrest was insufficient to account for potential confounders.

The quality of CPR, whether or not it incorporated a mechanical device, with respect to depth, rate, consistency of compressions and interruptions, was not reported in nine of 11 studies. Chest compression fraction is the percentage of time during which providers perform chest compressions during a cardiac arrest. In the CIRC trial, CPR fraction was monitored consistently (for $96 \%$ of patients) in both the mechanical and manual groups (mechanical 75\% versus manual 79\%) (Wik 2014). By comparison, the LINC study only monitored $10 \%$ of patients with impedence, and reported similar CPR fractions (mechanical $84 \%$ versus manual 78\%) (Rubertsson 2014). Both are higher than the CPR fraction 
reported in the Hallstrom 2006 study (mechanical 59\% versus manual $60 \%$, reported for $52 \%$ and $45 \%$ of patients, respectively). It is recognised that this is an important potential confounder in all cardiac arrest trials, thus the risk of bias for these studies is unclear. The quality of CPR received by participants in the manual CPR group is of paramount importance when assessing the results of comparative analyses. Substandard manual CPR administered by poorly trained providers during a study might inflate the measured relative treatment effect associated with the use of a mechanical CPR device in comparison to the treatment effect observed in a study with higher quality CPR in the manual chest compression control arm.

\section{Effects of interventions}

See: Summary of findings for the main comparison Mechanical chest compressions compared to manual chest compressions for cardiac arrest

A summary of the data from included studies can be found in Summary of findings for the main comparison. We did not pool data because of substantial clinical heterogeneity in the studies, including in the type of device (Thumper, pneumatic vest and LUCAS), participant (in-hospital, out-of-hospital), timing of device use (after failed conventional CPR versus during first attempts at CPR) and year of publication (studies span 1993 to 2017). Year of publication is important to consider with respect to clinical heterogeneity because recommendations for CPR, including the nature of manual chest compression, have changed drastically over the past 20 years (Perkins 2015a).

We assessed the quality of evidence for each outcome using the GRADE system (GRADEpro GDT 2015), with emphasis on the domains for risk of bias, inconsistency, indirectness, imprecision, and publication bias. For all outcomes, we did not deem that there was evidence of serious risk of indirectness.

\section{Mechanical chest compressions compared to manual chest compressions for cardiac arrest}

\section{Survival to hospital discharge with good neurological function}

There was moderate-quality evidence (downgraded for serious risk of bias) for the outcome of survival to hospital discharge with good neurological function (primary outcome of this review) reported by three studies involving 7587 patients (Hallstrom 2006; Rubertsson 2014; Wik 2014). Only the Hallstrom 2006 study observed a notable decrease in survival to hospital discharge with good neurological function (defined as Cerebral Performance Category score (CPC) 1 or 2) with the use of mechanical chest compressions as compared with manual chest compressions $(3.1 \%$ versus $7.5 \%$; risk ratio (RR) $0.41,95 \%$ confidence interval $(\mathrm{Cl}) 0.21$ to 0.79 ) (Analysis 1.1). The LINC trial did not demonstrate a difference between treatment groups for this outcome (CPC 1 or 2; mechanical $8.3 \%$ versus manual $7.8 \%$; RR $1.07,95 \% \mathrm{Cl} 0.82$ to 1.39 ) while the CIRC trial (modified Rankin Scale (mRS) 0 to 3; mechanical $4.1 \%$ versus manual $5.3 \%$; RR $0.79,95 \% \mathrm{Cl} 0.60$ to 1.04 ) demonstrated equivalence between the two arms (Rubertsson 2014; Wik 2014). The adjusted odds ratio for the mechanical compression arm versus the manual compression arm was 1.06 with a $95 \% \mathrm{Cl}$ of 0.83 to 1.37 which lay fully within the predefined equivalence region (Wik 2014).

\section{Survival to hospital discharge}

There was moderate-quality evidence (downgraded for serious risk of bias) for the outcome of survival to hospital discharge reported in seven studies involving 8067 patients (Gao 2016; Hallstrom 2006; Lu 2010; Rubertsson 2014; Smekal 2011; Taylor 1978; Wik 2014). Two studies using the LUCAS device (Rubertsson 2014; Smekal 2011), one study using a piston device (Taylor 1978), and one study using a load-distributing band device (Wik 2014), did not show harm or benefit when compared with manual compressions (Rubertsson 2014: 9.0\% versus 9.15\%; RR 0.98, 95\% Cl 0.77 to 1.25 ; Smekal 2011: $8.0 \%$ versus $9.72 \%$; RR $0.82,95 \% \mathrm{Cl} 0.29$ to 2.33 ; Taylor $1978: 12.5 \%$ versus 7.7\%; RR 1.63, 95\% $\mathrm{Cl} 0.30$ to 8.90 ; Wik 2014: 9.34\% versus $10.93 \%$; RR $0.85,95 \% \mathrm{Cl} 0.71$ to 1.02 ). Two studies, one using a piston for in-hospital cardiac arrest (IHCA) (Lu 2010), and the other using the AutoPulse for out-of-hospital cardiac arrest (OHCA) (Gao 2016), showed benefit of mechanical compressions (Lu 2010:32.9\% versus $14.7 \%$; RR 2.21, $95 \% \mathrm{Cl} 1.18$ to 4.17 ; Gao 2016: $18.8 \%$ versus $6.3 \%$; RR 3.01, $95 \% \mathrm{Cl} 1.04$ to 8.77 ).

Only one study favoured manual compressions (Hallstrom 2006). Hallstrom and colleagues generated an odds ratio (OR) 0.56 (95\% $\mathrm{Cl} 0.31$ to 1.00$)$ favouring manual chest compressions using multivariate logistic regression that adjusted for the clustering effect, age, initial rhythm, witness status, emergency medical services site, response time of first vehicle and location of arrest (public versus private) (Hallstrom 2006). (Note: this OR generated by the authors of the Hallstrom study using logistic regression differs slightly from that generated in Analysis 1.2 of this review using raw proportions abstracted from the published manuscript).

\section{Return of spontaneous circulation}

There was low-quality evidence (downgraded for serious risk of bias and inconsistency) for the outcome of return of spontaneous circulation reported by eight studies involving 11,771 patients (Dickinson 1998; Gao 2016; Halperin 1993; Lu 2010; Perkins 2015; Rubertsson 2014; Smekal 2011; Wik 2014; Analysis 1.3). Three studies $(\mathrm{N}=300)$ showed benefit with mechanical chest compressions compared with manual compressions (Dickinson 1998: $14.3 \%$ versus $0 \%$; RR 4.13, 95\% Cl 0.19 to 88.71 ; Lu $2010: 55.3 \%$ versus $37.8 \%$; RR $1.46,95 \% \mathrm{Cl} 1.02$ to 2.08 ; Gao $2016: 44.9 \%$ versus 23.4\%; RR $1.92,95 \% \mathrm{Cl} 1.15$ to 3.21$)$. Four studies ( $\mathrm{N}=7240)$ showed neither harm nor benefit (Halperin 1993: $47.1 \%$ versus $17.6 \%$; RR 2.67, $95 \% \mathrm{Cl} 0.85$ to 8.37 ; Perkins $2015: 31.6 \%$ versus $31.4 \%$; RR 1.01 , $95 \% \mathrm{Cl} 0.92$ to 1.10 ; Rubertsson 2014: $35.4 \%$ versus $34.6 \%$; RR 1.02 , $95 \% \mathrm{Cl} 0.92$ to 1.14; Smekal 2011: $40.5 \%$ versus 31.9\%; RR 1.27, $95 \%$ $\mathrm{Cl} 0.82$ to 1.96). In the large CIRC trial of 4231 patients, the Wik 2014 study calculated a relative risk, which was not adjusted for their interim analysis, of 0.88 ( $95 \% \mathrm{Cl} 0.81$ to 0.97 ), thus favouring manual chest compressions for return of spontaneous circulation.

\section{Survival to hospital admission}

There was moderate-quality evidence (downgraded for serious risk of bias) for the outcome of survival to hospital admission reported by four studies involving 7224 patients (Dickinson 1998; Perkins 2015; Rubertsson 2014; Smekal 2011; Analysis 1.4). No studies showed a difference in survival to hospital admission (Dickinson 1998: $14.3 \%$ versus $0 \%$; RR 4.13, $95 \%$ Cl 0.19 to 88.71 ; Perkins 2015: $22.8 \%$ versus $23.3 \%$; RR $0.98,95 \% \mathrm{Cl} 0.87$ to 1.09 ; Rubertsson 2014: $28.2 \%$ versus $27.7 \%$; RR $1.02,95 \% \mathrm{Cl} 0.90$ to 1.15 ; Smekal $2011: 24 \%$ versus $20.8 \%$; RR $1.15,95 \% \mathrm{Cl} 0.63$ to 2.11 ). 


\section{Survival to other time points}

The Perkins 2015 study reported ORs (adjusted for age, sex, response time, bystander CPR and initial rhythm, but not clustering design) for survival to three months with good neurological function (OR $0.72,95 \% \mathrm{Cl} 0.52$ to 0.99 ), suggesting harm associated with mechanical chest compression, while survival to three months (OR $0.83,95 \% \mathrm{Cl} 0.61$ to 1.12 ) and to 12 months (OR $0.83,95 \% \mathrm{Cl}$ 0.61 to 1.11 ) did not provide evidence for a difference associated with mechanical chest compression. Similarly, the Rubertsson 2014 study reported absolute risk differences (RDs) and found no difference in survival to one month ( $\mathrm{RD} 0.16,95 \% \mathrm{Cl}-2.0$ to 2.3 ) and six months (RD $0.47,95 \% \mathrm{Cl}-1.7$ to 2.6 ).

Among the included studies, a variety of other endpoints were reported for short-term survival ( $<30$ days). In one trial, the point estimate for survival to 24 hours mildly favoured manual compressions (adjusted OR $0.86,95 \% \mathrm{Cl} 0.74$ to 0.998 ) but the upper limit of the $95 \% \mathrm{Cl}$ was very close to unity (Wik 2014). No difference was found in four-hour survival by either the Rubertsson 2014 study (23.6\% versus $23.7 \%$; $\mathrm{P}<0.99,95 \% \mathrm{Cl}-3.3 \%$ to $3.2 \%$ ) or the Hallstrom 2006 study $(26.4 \%$ versus $24.7 \%$; reported $P=0.62)$.

\section{Adverse effects}

Several included studies reported adverse effect and time interval data, but we did not conduct pooled analyses were not done because of considerable clinical heterogeneity. Specifically, the type of device and the mechanism of the device were thought to be of paramount relevance to injury patterns observed in treated participants.

There was low-quality evidence (downgraded for serious risk of bias and imprecision) for the outcome of sternal or rib fractures reported by seven studies involving 7469 patients (Gao 2016; Halperin 1993; Koster 2017; Lu 2010; Rubertsson 2014; Taylor 1978; Wik 2014). Point estimates for RRs derived from the data reported in these studies were divergent. Data from the Wik 2014 study suggested a two-fold increase in the risk for rib or sternal fractures with mechanical chest compressions compared to manual compressions (RR 1.98, 95\% Cl 1.33 to 2.94; Analysis 1.5).

For the outcome of pneumothorax or haemothorax, there was low-quality evidence (downgraded for serious risk of bias and imprecision) reported by five studies involving 7316 patients (Halperin 1993; Koster 2017; Lu 2010; Rubertsson 2014; Wik 2014). Of these, three studies had very wide Cls due to the low number of events (Analysis 1.6).

For the outcome of other internal organ injury, there was low-quality evidence (downgraded for serious risk of bias and imprecision) reported by five studies involving 7337 patients (Koster 2017; Lu 2010; Rubertsson 2014; Taylor 1978; Wik 2014; Analysis 1.7).

The Koster 2017 study deserves special mention here because it was designed with injury as the primary outcome. This was a three-arm randomised controlled non-inferiority trial with a primary outcome of "serious or life-threatening visceral resuscitation-related damage" detected by post-resuscitation computed tomography (CT) scan, autopsy and clinical follow-up. 'Life-threatening' was defined as "reasonably expected to interfere with cardiovascular or respiratory function, exsanguination in excess of $800 \mathrm{~mL}$ " and 'serious' was defined as "demands therapy for repair or for alleviation of pain, expected to prolong hospitalisation". Patients with OHCA or IHCA were randomised to receive standard manual compressions, AutoPulse compressions or LUCAS compressions. The non-inferiority margin was set a priori to be $<10 \%$ excess serious or life-threatening resuscitationrelated injury. The primary outcome was determined by a panel of clinicians using all available information. Blinding outcome assessors was attempted, but it was admitted by the study authors that the mechanical devices often left characteristic marks on the chest that may have undermined attempts. It was not clear whether outcome assessors were blinded to study design (e.g. non-inferiority trial) which is important to avoid bias and a type I error in non-inferiority studies (New Reference). The risk difference for AutoPulse compared to manual compressions for the primary outcome was $+5.3 \%(95 \% \mathrm{Cl}-2.2 \%$ to $12.8 \%$; $\mathrm{P}=0.15)$ which did not satisfy criteria for non-inferiority. The risk difference for LUCAS compressions compared to manual compressions for the primary outcome was $+1.0 \%(95 \% \mathrm{Cl}-5.5 \%$ to $7.6 \% ; \mathrm{P}=0.75$ ), which met non-inferiority criteria. The study authors concluded that LUCAS compressions do not cause significantly more serious or life-threatening visceral damage than manual chest compressions. They also concluded that for AutoPulse, significantly more serious or life-threatening visceral damage than manual chest compressions cannot be excluded.

\section{Time interval data}

Time interval data were reported by four studies (Hallstrom 2006; Rubertsson 2014; Smekal 2011; Wik 2014). In three studies, there was a delay in the first shock with the mechanical compression group compared to the manual compression group. In the Hallstrom 2006 trial, time from the emergency call to first rhythm analysis was similar in the two groups $(8.9 \pm 3.0$ minutes versus $8.9 \pm 2.9$ minutes), but the emergency call to first shock interval in those participants with a shockable rhythm was longer in the mechanical chest compression group than in the manual chest compression group ( $11.8 \pm 6.1$ minutes versus $9.7 \pm 3.1$ minutes, reported $P=0.001)$. In the LINC trial, intervals were measured from the estimated time of cardiac arrest (Rubertsson 2014). The median interval between cardiac arrest and emergency call was two minutes (interquartile range (IQR) 0 to 5 ) in both arms. Notably, there was a 1.5-minute delay in time from cardiac arrest to first defibrillation in the mechanical group (17, IQR 12 to 22) compared to the manual group (15.5, IQR 11 to 23.5 ). This delay may be at least partly explained by the protocol which states that the first shock was to be delivered at 90 seconds after the start of mechanical CPR without a rhythm check. In the CIRC trial, the average interval from scene arrival to first shock for patients in ventricular fibrillation/ ventricular tachycardia was slightly longer for the mechanical arm (7.5 \pm 6.0 minutes versus $6.7 \pm 6.2$ minutes) (Wik 2014).

The Smekal 2011 trial reported similar scene arrival time to first CPR interval ( $1.0 \pm 1.1$ minutes versus $1.1 \pm 1.1$ minutes). The Smekal 2011 trial also reported cardiac arrest time to start of LUCAS device (13.1 \pm 7.2 minutes), cardiac arrest time to start of CPR $(10.4 \pm 6.6$ minutes versus $10.2 \pm 5.9$ minutes) and call from dispatch centre to start of CPR ( $8.3 \pm 5.8$ minutes versus $7.5 \pm 3.6$ minutes).

\section{Subgroup analyses}

\section{Initial cardiac arrest rhythm}

We planned a subgroup analysis on the basis of initial cardiac arrest rhythm, however, only two of the included studies reported 
outcomes by initial cardiac arrest rhythm (Hallstrom 2006; Perkins 2015). We did not undertake a pooled analysis because of insufficient data and significant heterogeneity. In the Hallstrom 2006 study, four-hour survival was higher in the mechanical group as compared to the manual group (17.2\% versus $10.4 \%)$. This difference was labelled as a "trend" by authors in the absence of reporting any statistical hypothesis testing being reported in the manuscript. In the Perkins 2015 study, survival was lower among those treated with a mechanical chest compression device compared with those treated with only manual chest compressions in the subgroup of patients having ventricular fibrillation or ventricular tachycardia as their initial cardiac arrest rhythm (OR $0.71,95 \% \mathrm{Cl} 0.52-0.98)$. There was no difference between treatment groups in the subgroup of patients with pulseless electrical activity (OR $1.38,95 \% \mathrm{Cl} 0.80-2.36$ ).

\section{DISCUSSION}

\section{Summary of main results}

After an extensive search, we identified 11 randomised trials, with data from 12,944 participants, which included 10 randomised controlled trials (RCTs) (8 randomised at the patient level and two cluster-randomised) and one quasi-randomised trial. The publication dates of these studies span five decades (1978 to 2015), and results demonstrate marked heterogeneity in participant selection (out-of-hospital, in-hospital), cardiac arrest aetiologies, timing of device application (immediate versus delayed after failed traditional cardiopulmonary resuscitation (CPR)), types of mechanical devices used and control group CPR protocols.

This update has added three large RCTs (which account for $90 \%$ of all patients in this review). The primary outcome of this review, survival to hospital discharge with good neurological function, has only been reported for out-of-hospital cardiac arrest (OHCA) in the LINC, CIRC and Hallstrom studies (Hallstrom 2006; Rubertsson 2014; Wik 2014). In contrast to the previous results by Hallstrom et al, which suggested that mechanical compressions may be harmful, the new data from the CIRC and LINC trials showed no difference in survival to discharge with good neurological function (Rubertsson 2014; Wik 2014). The CIRC study concluded equivalence between mechanical and manual arms with respect to this outcome (Wik 2014). There was no evidence of a difference between treatment arms in short-term ( $<30$ days) survival reported in the CIRC, LINC and PARAMEDIC trials (Perkins 2015; Rubertsson 2014; Wik 2014; Characteristics of included studies). However, the PARAMEDIC study did report an adjusted OR for neurologically favourable survival at three months, suggesting superiority of manual chest compressions (Perkins 2015). None of the other large clinical trials reported strong evidence for differences in long-term outcomes. There is no strong evidence to suggest that mechanical chest compression devices cause an excess of adverse effects compared with manual chest compressions, however, most studies included in this review did not report a robust methodology for identifying CPR-related injuries in either arm.

The bulk of available evidence from RCTs included in this review would suggest that CPR protocols involving mechanical chest compression devices produce similar clinical outcomes compared to manual CPR protocols involving very high-quality chest compressions. This finding seems to conflict with the preclinical and observational data demonstrating that mechanical devices can provide superior compressions with respect to rate, depth and consistency resulting in superior haemodynamic effects compared to manual compressions. Many may ask why these findings have not translated into improved clinical outcomes in published randomised clinical trials.

It is possible that the superiority of mechanical devices observed in previous observational studies was spurious and the manifestation of bias. Most importantly, the issue of selection bias needs to be considered. Where patients were not randomised, they may have been selected for mechanical chest compression device use by healthcare providers based on characteristics (measured or unmeasured) favourable for good outcome.

Integration of mechanical devices into the resuscitation algorithms of included studies may have had negative impacts on overall CPR quality (e.g. pauses in chest compressions during device deployment and delayed first defibrillation in patients with ventricular fibrillation) negating any physiologic benefit observed in preclinical studies. Time to first defibrillation was longer in the mechanical chest compression arms of several studies included in this review (Hallstrom 2006; Rubertsson 2014; Wik 2014).

Consideration of the control groups is paramount in interpreting the results of these studies. Conclusions of superiority or equivalence of mechanical chest compression devices to manual chest compressions are entirely dependent on the quality of resuscitation provided in the manual chest compression group. All else being the same, a study with poor-quality manual compressions is more likely to favour mechanical devices. The Wik 2014 study, for example, involved significant training and careful monitoring of CPR quality in both arms; CPR fraction in the control arm was $79 \%$. The equivalence conclusion of this study needs to be carefully qualified because the comparison group received very high-quality manual chest compressions. Had the quality of manual chest compressions been lower, the results may have been different. Similarly, the LINC trial demonstrated a CPR fraction of $78 \%$ in the manual chest compression arm (Rubertsson 2014). This high-quality manual chest compression is likely not reflective of most emergency medical services systems in the world, with many prior studies reporting much lower CPR fractions closer to $50 \%$ (Wik 2005). Therefore, the relative benefit of implementing mechanical chest compression devices may be different than that demonstrated by the trials included in this review, reporting high CPR quality in the control group.

Improved haemodynamics associated with the use of mechanical chest compression devices may not translate into improved clinical outcomes for several reasons. Although we did not identify any evidence to suggest an overall excess of injuries related to the use of mechanical chest compression devices, there may have been unmeasured and unreported complications from mechanical chest compression devices such as rib, lung, cardiac or intraabdominal injuries that may have negated the impact of any improvement in haemodynamics during cardiac arrest associated with mechanical devices. As with many other advanced therapies for cardiac arrest, they are often implemented with some delay after the occurrence of cardiac arrest. Perhaps the window of opportunity for improved haemodynamics to impact outcomes is closed by the time mechanical chest devices can be reasonably implemented in most cardiac arrests. 


\section{Overall completeness and applicability of evidence}

The completion of the CIRC, LINC and PARAMEDIC trials brings in results backed by large sample sizes that more thoroughly address the primary and secondary outcomes defined in this review. With these studies' inclusion, the vast majority of the participants are OHCA patients. We made the decision to exclude non-randomised studies because of their vulnerability to additional biases, including differences in quality of care between crews and emergency medical services systems (non-randomised concurrent controls), changes in practice and in quality of care over time (historical controls), the Hawthorne effect (historical or concurrent controls) and selection bias (preferential application of a device to participants thought to have a very poor prognosis in the hope that use of the device might lead to better outcomes than are attained with standard care). Most studies have focused on two mechanical devices, the LUCAS device and the AutoPulse. To date, four trials are still in progress and have not yet been published (CTRI/2013/07/003840; ISRCTN38139840; ISRCTN78354073; NCT01521208).

Certain potentially important patient subgroups were either underrepresented or not reported. For instance, there were less females in the studies included in our review and body mass index was under-reported. There may be important differences in the effectiveness of mechanical chest compression devices in these important and under-represented subgroups (such as females and morbidly obese), but the data did not allow such subgroup analysis. Generalisability of our results for these important subgroups of patients is unclear.

The data identified in this review did not support a robust subgroup analysis of outcomes on the basis of initial cardiac rhythm. This is a major limitation of the data because defibrillation is a crucial and time-dependent element of treatment for patients with shockable initial rhythms. The use of mechanical chest compression devices and their effect on defibrillation must be carefully considered. Only two of the studies included in the review reported outcomes by initial cardiac arrest rhythm (Hallstrom 2006; Perkins 2015). Although this requires further investigation to confirm, results from both of these studies suggest that mechanical chest compression devices may be more beneficial in patients with non-shockable rhythms. The differential effect in rhythm subgroups may relate to delayed defibrillation or some other unmeasured effect of these machines on defibrillation (e.g. pad placement). Further evidence is required on the relative efficacy of mechanical chest compression devices in patients with different initial rhythms.

None of the included studies reported outcomes by response time interval. It may be that mechanical chest compression devices have a differential effect among patients treated earlier in the cardiac arrest as opposed to later in the cardiac arrest. Time to defibrillation among those with shockable rhythms and reduced no-flow time may relate to shorter response time intervals. Available data are unable to shed light on whether mechanical chest compression devices may be more or less effective at different time points along the timeline from collapse to resuscitation initiation.

\section{Quality of the evidence}

The quality of the evidence for each outcome ranged from low to moderate quality, assessed using the GRADE approach (GRADEpro GDT 2015) (Summary of findings for the main comparison).

\section{Limitations in study design - risk of bias}

The methods of randomisation and allocation concealment were unclear in the majority of studies and we assessed all studies as unclear with regard to selective reporting (Figure 2; Figure 3).

CPR algorithms for both the manual and mechanical compression groups have been described in the new clinical trials added since our last update. The lack of CPR process and quality description in the older studies has been highlighted as a major methodological limitation and potential source of bias in previous versions of this review. These descriptions highlight a number of notable differences in how CPR was delivered across studies.

The Smekal 2011 trial is subject to problems with the cointervention, as a modified CPR algorithm was used in the mechanical chest compression group. The much larger LINC trial by the same authors also used a study-specific algorithm allowing cointervention in the mechanical group where defibrillation was given without rhythm analysis (Rubertsson 2014). The manual CPR group also followed the outdated 2005 European Resuscitation Guidelines. It is difficult to judge how much of the observed treatment effect could be explained by a difference in CPR process rather than by use of the chest compression device alone.

In contrast to the focus on high-quality CPR through training programmes and continuous monitoring of compliance with mechanical device deployment in the CIRC trial, the PARAMEDIC study was designed as a pragmatic trial. As such, they implemented mechanical CPR training according to routine organisation practices (Perkins 2015). It is unclear how much oversight was present to ensure compliance given that $40 \%$ of the group assigned to the mechanical chest compression group received manual compressions only. This may be a reflection of real-world challenges in deploying the device. There may be unwillingness among emergency medical services providers to deploy the device on the basis of prejudice (i.e. a belief that manual compressions are superior) or inconvenience. Thus, their results may be interpreted to either reflect an underestimate of any true treatment effect associated with mechanical CPR (efficacy) or an accurate estimate of the effectiveness of mechanical CPR when deployed in the real world.

The Hallstrom 2006 study, which suggested an association between the use of mechanical chest compression devices and worse outcomes, was at risk for bias in several forms. Specifically, the mechanical chest compression group, although balanced with respect to the number of people with ventricular fibrillation or pulseless ventricular tachycardia, had an average 2.1 minutes extra delay to first defibrillation attempt. Because three options were available for integration of the device into the CPR protocol, and because complete CPR process data were lacking, it is unclear whether this delay was a result of time needed for device deployment and to what degree this delay could have been reduced by improved training. It is plausible that this delay is partially, if not completely, responsible for the differences in outcomes between the two groups. A reanalysis of the original trial data has demonstrated that the harm associated with mechanical chest compressions in the analysis was entirely dependent on data arising from the single site (site $\mathrm{C}$ ) that incorporated a protocol change part-way through the study, which entailed delayed deployment of the device during the resuscitation sequence (Paradis 2010). Although evidence for differential effects of the 
AutoPulse device on participants with different body types is not known to the authors of this review, it is possible that the excess of extreme body types in the mechanical CPR group ("thin" and "morbidly obese") may have had an impact on the efficacy of the device that was unaccounted for in the analysis. Prestudy outcomes at each of the participating sites were not reported, so it is difficult to assess whether a Hawthorne effect may have exerted a positive influence on the quality of manual chest compressions in such a way as to make it more challenging for the mechanical chest compression arm to show superiority. Data from the Hallstrom 2006 study highlight the potential importance of device deployment with respect to optimising clinical outcomes.

The CIRC study (Wik 2014), which is the largest and most robust of the identified trials, was designed to overcome some of the limitations of the Hallstrom 2006 study. Specifically, the study protocol involved consistent CPR monitoring (by transthoracic impedance or accelerometer date) and three study phases (infield training, run-in and statistical inclusion) to minimise biases and learning effects (Wik 2014). Wik and colleagues noted a higher than normal CPR quality which drew them to the conclusion that mechanical CPR was statistically equivalent to high-quality manual CPR.

Several studies did not adequately describe participants included in the study with respect to important covariates known to be associated with survival. For instance, the Lu 2010 study did not report the initial rhythm for included participants. Imbalance between treatment groups with respect to this important prognostic factor may have contributed to observed differences in return of spontaneous circulation.

\section{Inconsistency of results}

We did not pool studies on the basis of significant clinical heterogeneity among included studies with regards to population and intervention characteristics. $\left.\right|^{2}$ values for all survival outcomes demonstrated signficant statistical heterogeneity (64\% to $75 \%$ ). Based on assessment of non-overlapping confidence intervals and variance of point estimates for the outcome, return of spontaneous circulation, we downgraded it for inconsistency.

\section{Indirectness of evidence}

Although there was some indirectness across studies, we did not feel that there was enough to downgrade our quality of evidence assessments for any of the outcomes. Some studies provided evidence that was indirect with respect to the population studied. For example, the Halperin 1993 study included patients who had failed conventional resuscitation. These patients were much further along in the process towards irreversible death than our target population for this intervention. Some studies provided evidence that was indirect by way of the intervention studied. For instance, some of the devices studied are not commercially available and mechanistically different to those available (e.g. the vest device in the Halperin study).

\section{Imprecision}

Imprecision is indicated in several outcomes by excessively wide confidence intervals. This is most pronounced in the outcomes related to adverse events. We downgraded each of the adverse events outcomes for imprecision on this basis.

\section{Publication bias}

We have no strong evidence for publication bias and did not downgrade quality assessments on this basis for any of the outcomes assessed. We felt that there were too few studies for funnel plots to be reliable. We found no evidence of unpublished studies in our assessment of clinical trial registries.

\section{Potential biases in the review process}

The strengths of this review lie in the comprehensive search of the literature involving several databases, handsearching and contact with experts and industry contacts. Two independent investigators completed reviews for inclusion and data abstraction, reducing the opportunity for some forms of measurement bias.

The decision to limit the content of the review to randomised studies means that we may have missed some important data from non-randomised studies. Our search strategy may have missed some unpublished studies because it focused on databases that primarily include published work.

\section{Agreements and disagreements with other studies or reviews}

Since the last update, there have been several additional metaanalyses beyond the Westfall 2013 review that take the CIRC, LINC and PARAMEDIC trials into account (Gates 2015; Tang 2015). We determined at the outset of the original review to not pursue pooling of data if either the descriptive clinical heterogeneity or the statistical heterogeneity were too substantial $(12>50 \%)$. Nonetheless, the cumulative data described in this review are in agreement with these other reviews; namely, that mechanical compressions do not offer a short- or long-term survival advantage when compared with manual compressions and may be equivalent to CPR with high-quality manual compressions.

\section{AUTHORS' CONCLUSIONS}

\section{Implications for practice}

The evidence does not suggest that CPR protocols involving mechanical chest compression devices are superior to conventional therapy involving manual chest compressions only. We conclude on the balance of evidence that mechanical chest compression devices used by trained individuals are a reasonable alternative to manual chest compressions in settings where consistent, high-quality manual chest compressions are not possible or dangerous for the provider (eg, limited rescuers available, prolonged CPR, during hypothermic cardiac arrest, in a moving ambulance, in the angiography suite, during preparation for extracorporeal CPR [ECPR], etc.). Systems choosing to incorporate mechanical chest compression devices should be closely monitored because some data identified in this review suggested harm. Special attention should be paid to minimising time without compressions and delays to defibrillation during device deployment.

\section{Implications for research}

Future research should study the effect of mechanical chest compression devices in special scenarios where sustainable highquality manual chest compressions are challenging or pose a risk to providers. Examples of these scenarios include CPR in a moving ambulance, situations where limited rescuers are available, 
during prolonged resuscitations (e.g. accidental hypothermia, toxicological causes), in the angiography suite or during the implementation of extracorporeal cardiopulmonary resuscitation. The relative effect of mechanical chest compressions in important subpopulations requires investigation. For example, patients with non-shockable initial cardiac arrest rhythms may benefit more from mechanical compressions when defibrillation is not indicated and there are no concerns about delay to successful defibrillation associated with implementation of devices.

There is a paucity of data from direct comparisons of different devices with respect to ease of implementation and patient safety. Improved methods of implementation and deployment of the devices which minimise no flow time and delays to defibrillation could be developed. Any research involving mechanical chest compression devices should include the accurate measurement of CPR process in all arms of the study. Data on the use of mechanical chest compression devices in the paediatric population is scant and should be the focus of future investigations.

\section{ACKN OWLEDGEMENTS}

The authors would like to thank Dr Prakeshkumar Shah and Dr Joseph Bayene at the University of Toronto for their input and feedback on the original protocol. Thank you also to members of the Cochrane Heart Group internal review for their advice and feedback. Thank you to Ms Margaret Burke, Trials Search Coordinator of the Cochrane Heart Group, for assistance with the development of search strategies for the original review.

We acknowledge the contributions of co-authors on previous versions of this review. Nizar Hassan contributed to the 2013 update. He conducted quality review, data abstraction, data analysis, manuscript update drafting and editing. Blair Bigham was involved with the development of the original protocol and updates up to 2014, including selection of studies, quality review, data analysis and manuscript draft editing. Laurie Morrison participated in development of the original protocol and updates up to 2014, including data abstraction, data analysis and manuscript draft edits.

We would also like to express our gratitude for the translation services of Isaac Fung and Xuran Jiang. The authors would like to acknowledge Cochrane staff, including Nicole Martin and Fiona Taylor, for their assistance in co-ordinating the updated searches and translation. Finally, we would like to thank all of the anonymous Cochrane reviewers for their careful edits and feedback which have made this review stronger. 


\section{RE F E R E N C E S}

\section{References to studies included in this review}

Dickinson 1998 \{published data only\}

Dickinson ET, Verdile VP, Schneider RM, Salluzzo RF. Effectiveness of mechanical versus manual chest compressions in out-of-hospital cardiac arrest resuscitation: a pilot study. American Journal of Emergency Medicine 1998;16(3):289-92.

\section{Gao 2016 \{published data only\}}

Gao C, Chen Y, Peng H, Chen Y, Zhuang Y, Zhou S. Clinical evaluation of the AutoPulse automated chest compression device for out-of-hospital cardiac arrest in the northern district of Shanghai, China. Archives of Medical Science 2016;12(3):563-70.

\section{Hallstrom 2006 \{published data only\}}

Hallstrom A, Rea TD, Sayre MR, Christenson J, Anton AR, Mosesso VN Jr, et al. Manual chest compression vs use of an automated chest compression device during resuscitation following out-of-hospital cardiac arrest: a randomized trial. JAMA 2006;H295(22):2620-8.

\section{Halperin 1993 \{published data only\}}

Halperin HR, Tsitlik JE, Gelfand M, Weisfeldt ML, Gruben KG, Levin HR, et al. A preliminary study of cardiopulmonary resuscitation by circumferential compression of the chest with use of a pneumatic vest. New England Journal of Medicine 1993;329(11):762-8.

\section{Koster 2017 \{published data only\}}

Koster RW, Beenen LF, van der Boom EB, Spijkerboer AM, Tepaske R, van der Wal AC, et al. Safety of mechanical chest compression devices AutoPulse and LUCAS in cardiac arrest: a randomized clinical trial for non-inferiority. European Heart Journal 2017;38(40):3006-13. [DOI: 10.1093/eurheartj/ehx318]

\section{Lu 2010 \{published data only\}}

Lu XG, Kang X, Gong DB. The clinical efficacy of Thumper modal 1007 cardiopulmonary resuscitation: a prospective randomized control trial. Zhongguo Wei Zhong Bing Ji Jiu Yi Xue 2010;22(8):496-7.

\section{Perkins 2015 \{published data only\}}

Perkins GD, Lall R, Quinn T, Deakin CD, Cooke MW, Horton J, et al. Mechanical versus manual chest compression for outof-hospital cardiac arrest (PARAMEDIC): a pragmatic, cluster randomised controlled trial. Lancet 2015;385(9972):947-55.

\section{Rubertsson 2014 \{published data only\}}

Hardig BM, Lindgren E, Östlund O, Herlitz J, Karlsten R, Rubertsson S. Outcome among VF/VT patients in the LINC (LUCAS IN cardiac arrest) trial-A randomised, controlled trial. Resuscitation 2017;115:155-62.

* Rubertsson S, Lindgren E, Smekal D, Ostlund O, Silfverstolpe J, Lichtveld RA, et al. Mechanical chest compressions and simultaneous defibrillation vs conventional cardiopulmonary resuscitation in out-of-hospital cardiac arrest: the LINC randomized trial. JAMA 2014;311(1):53-61.
Smekal 2011 \{published data only\}

* Smekal D, Johansson J, Huzevka T, Rubertsson S. A pilot study of mechanical chest compressions with the LUCAS ${ }^{T M}$ device in cardiopulmonary resuscitation. Resuscitation 2011;82(6):702-6.

Smekal D, Lindgren E, Sandler H, Johansson J, Rubertsson S. CPR-related injuries after manual or mechanical chest compressions with the LUCAS device: A multicentre study of victims after unsuccessful resuscitation. Resuscitation 2014;85(12):1708-12.

Taylor 1978 \{published data only\}

Taylor GJ, Rubin R, Tucker M, Greene HL, Rudikoff MT, Weisfeldt ML. External cardiac compression. A randomized comparison of mechanical and manual techniques. JAMA 1978;240(7):644-6.

\section{Wik 2014 \{published data only\}}

* Wik L, Olsen J A, Persse D, Sterz F, Lozano M, Brouwer M A, et al. Manual vs. integrated automatic load-distributing band CPR with equal survival after out of hospital cardiac arrest. The randomized CIRC trial. Resuscitation 2014;85(6):741-8.

Wik L, Olsen JA, Persse D, Sterz F, Lozano Jr M, Brouwer MA et al. Comparison of survival to hospital discharge between integrated autopulse-CPR and manual-CPR during outof-hospital cardiac arrest of presumed cardiac origin: the circulation improving resuscitation care (CIRC) trial. Circulation 2011;21:2374.

Wik L, Olsen JA, Persse D, Sterz F, Lozano M Jr, Brouwer MA, et al. Corrigendum to "Manual vs. integrated automatic loaddistributing band CPR with equal survival after out of hospital cardiac arrest. The randomized CIRC trial" [Resuscitation 85 (2014) 741-8]. Resuscitation 2014;85(9):1306.

\section{References to studies excluded from this review}

Anonymous 1973 \{published data only\}

Anonymous. External cardiac compressors. Health Devices 1973;2(6):136-48 passim.

\section{Arntz 1998 \{published data only\}}

Arntz HR. New developments in non-invasive mechanical cardiac life support. Acta Anaesthesiologica Scandinavica 1998;42:101-3.

\section{Arntz 2001 \{published data only\}}

Arntz HR, Agrawal R, Richter H, Schmidt S, Rescheleit T, Menges $\mathrm{M}$, et al. Phased chest and abdominal compressiondecompression versus conventional cardiopulmonary resuscitation in out-of-hospital cardiac arrest. Circulation 2001;104(7):768-72.

\section{Aufderheide 2011 ppublished data only\}}

Aufderheide TP, Frascone RJ, Wayne MA, Mahoney BD, Swor RA, Domeier RM, et al. Standard cardiopulmonary resuscitation versus active compression-decompression cardiopulmonary resuscitation with augmentation of negative intrathoracic 
pressure for out-of-hospital cardiac arrest: a randomised trial. The Lancet 2011;377:301-11.

\section{Axelsson 2006 \{published data only\}}

Axelsson C, Nestin J, Svensson L, Axelsson AB, Herlitz J. Clinical consequences of the introduction of mechanical chest compression in the EMS system for treatment of out-of-hospital cardiac arrest-a pilot study. Resuscitation 2006;71(1):47-55.

\section{Axelsson 2009 \{published data only\}}

Axelsson C, Karlsson T, Axelsson AB, Herlitz J. Mechanical active compression-decompression cardiopulmonary resuscitation (ACD-CPR) versus manual CPR according to pressure of end tidal carbon dioxide (PETCO2) during CPR in out-of-hospital cardiac arrest (OHCA). Resuscitation 2009;80(10):1099-103.

Baubin 1999 \{published data only\}

Baubin M, Sumann G, Rabl W, Eibl G, Wenzel V, Mair P. Increased frequency of thorax injuries with ACD-CPR. Resuscitation 1999;41(1):33-8.

\section{Box 2008 \{published data only\}}

Box MS, Watson JN, Addison PS, Clegg GR, Robertson CE. Shock outcome prediction before and after CPR: a comparative study of manual and automated active compression-decompression CPR. Resuscitation 2008;78(3):265-74.

\section{Dittbrenner 1993 \{published data only\}}

Dittbrenner H. CPR systems-resuscitation for health care records. Caring 1993;12(7):24-6.

\section{Dotter 1961 \{published data only\}}

Dotter CT, Straube KR, Strain DC. Circulatory arrest: manual and mechanical means for emergency management. Radiology 1961;77:426-33.

\section{Elich 1995 \{published data only\}}

Elich D, Kerz T, Dick W. Cardiopulmonary resuscitation. Current Opinion in Anaesthesiology 1995;8(2):152-6.

\section{Halperin 2000 \{published data only\}}

Halperin H, Berger R, Chandra N, Ireland M, Leng C, Lardo A, et al. Cardiopulmonary resuscitation with a hydraulic-pneumatic band. Critical Care Medicine 2000;28(11 Suppl):N203-6.

\section{Hampe 2008 \{published data only\}}

Hampe S, Dinse-Lambracht A. AutoPulse (R)-assisted CPR. New chances for patients with cardiac arrest?. Notfall \& Rettungsmedizin 2008;11(8):563-5.

\section{Harkins 1961 \{published data only\}}

Harkins GA, Bramson ML. Mechanized external cardiac massage for cardiac arrest and for support of the failing heart. Journal of Scientific and Industrial Research 1961;1:197-200.

\section{Havel 2008 \{published data only\}}

Havel C, Berzlanovich A, Sterz F, Domanovits H, Herkner H, Zeiner A, et al. Safety, feasibility, and hemodynamic and blood flow effects of active compression-decompression of thorax and abdomen in patients with cardiac arrest. Critical Care Medicine 2008;36(6):1832-7.
Kern 2001 \{published data only\}

Kern KB, Morley PT, Babbs CF, Halperin HR, de Latorre FJ, Lurie KG, et al. Use of adjunctive devices in cardiopulmonary resuscitation. Annals of Emergency Medicine 2001;37(4):S68-77.

Knight 1964 \{published data only\}

Knight ICS. New apparatus for intermittent cardiac compression. Britisih Medical Journal 1964;1:894.

Krischer 1989 \{published data only\}

Krischer JP, Fine EG, Weisfeldt ML, Guerci AD, Nagel E, Chandra N. Comparison of prehospital conventional and simultaneous compression-ventilation cardiopulmonary resuscitation. Critical Care Medicine 1989;17(12):1263-9.

\section{Kurowski 2015 \{published data only\}}

Kurowski A, Czyżewski L, Bogdański L, Zaśko P, Karczewska K, Szarpak L. Quality of chest compression with CardioPump CPR compared to single rescuer standard BLS. American Journal of Emergency Medicine 2015;33(1):114-5.

\section{Lairet 2005 \{published data only\}}

Lairet J, Lee M. A comparison of standard manual cardiopulmonary resuscitation versus the AutoPulse mechanical cardiopulmonary resuscitation device. Annals of Emergency Medicine 2005;46(3 Suppl):S114.

\section{Liu 2010 \{published data only\}}

Liu Q-Y, Li C-S. The effect of the external chest compression appliance (AutoPulse) on cardiac arrest in the emergency department and influence on blood gas and $\mathrm{N}$-terminal B-type natriuretic peptide. Chinese Critical Care Medicine 2010;22(11):660-2.

\section{Lurie 1994 \{published data only\}}

Lurie KG, Shultz JJ, Callaham ML, Schwab TM, Gisch T, Rector T, et al. Evaluation of active compression-decompression CPR in victims of out-of-hospital cardiac arrest. JAMA 1994;271(18):1405-11.

Lurie 1997 \{published data only\}

Lurie KG. Recent advances in mechanical methods of cardiopulmonary resuscitation. Acta Anaesthesiologica Scandinavica 1997;41:49-52.

Lurie 2002 \{published data only\} Lurie K. Mechanical devices for cardiopulmonary resuscitation: an update. Emergency Medicine Clinics of North America 2002;20(4):771-84.

\section{Malzer 1996 \{published data only\}}

Malzer R, Zeiner A, Binder M, Domanovits H, Knappitsch G, Sterz F, et al. Hemodynamic effects of active compressiondecompression after prolonged CPR. Resuscitation 1996;31(3):243-53.

\section{Montgomery 1995 \{published data only\}}

Montgomery WH. Mechanisms and methods of cardiopulmonary resuscitation. Anesthesiology Clinics of North America 1995;13(4):767-83. 


\section{Morozumi 2009 \{published data only\}}

Morozumi J, Sakurai E, Matsuno N, Ito M, Yokoyama T, Ohtaka Y, et al. Successful kidney transplantation from donation after cardiac death using a load-distributing-band chest compression device during long warm ischemic time. Resuscitation 2009;80(2):278-80

\section{Nachlas 1962 \{published data only\}}

Nachlas MM, Siedband MP. A simple portable pneumatic pump for external cardiac massage. American Journal of Cardiology 1962;10:107-9.

\section{Nachlas 1963 \{published data only\}}

Nachlas MM, Miller DI, Siedband MP. Determination of cardiorespiratory variables in experimental cardiac resuscitation using a mechanized pump for external cardiac massage. Annals of Surgery 1963;158:295-308.

Nachlas 1965 \{published data only\}

Nachlas MM. Clinical experiences with mechanical cardiac massage. American Journal of Cardiology 1965;15:310-9.

\section{Niemann 1984 \{published data only\}}

Niemann JT, Rosborough JP, Niskanen RA, Criley JM. Mechanical cough CPR during cardiac-arrest-improved coronary perfusion-pressure and long-term survival. Circulation 1984;70(4):383.

\section{Nishino 1992 \{published data only\}}

Nishino T, Ishikawa T, Tanaka A, Hiraga K. Respiratory responses to chest compression in human subjects. American Review of Respiratory Disease 1992;146(4):980-4.

\section{Pearson 1966 \{published data only\}}

Pearson JW, Navarro RN, Redding JS. Evaluation of mechanical devices for closed chest cardiac massage. Anesthesia \& Analgesia 1966;45:590-8.

\section{Plaisance 1999 \{published data only\}}

Plaisance P, Lurie KG, Vicaut E, Adnet F, Petit JL, Epain D, et al. A comparison of standard cardiopulmonary resuscitation and active compression-decompression resuscitation for out-of-hospital cardiac arrest. French Active CompressionDecompression Cardiopulmonary Resuscitation Study Group. New England Journal of Medicine 1999;341(8):569-75.

\section{Rivers 1993 \{published data only\}}

Rivers EP, Boczar ME, Smithline HA, Lurie KG, Nowak RM. A comparison of mechanical, standard and active compressiondecompression CPR in out-of-hospital cardiac-arrest patients. Circulation 1993;88(4):193.

\section{Roberts 1978 \{published data only\}}

Roberts BG, Bryan JM. Dallas EMS system advocates mechanical CPR. Emergency Medical Services 1978;7(4):39-40.

\section{Schwab 1995 \{published data only\}}

Schwab TM, Callaham ML, Madsen CD, Utecht TA. A randomized clinical trial of active compression-decompression CPR vs standard CPR in out-of-hospital cardiac arrest in two cities. JAMA 1995;273(16):1261-8.
Skogvoll 1999 \{published data only\}

Skogvoll E, Wik L. Active compression-decompression cardiopulmonary resuscitation: a population-based, prospective randomised clinical trial in out-of-hospital cardiac arrest. Resuscitation 1999;42(3):163-72.

Smekal 2009 \{published data only\}

Smekal D, Johansson J, Huzevka T, Rubertsson S. No difference in autopsy detected injuries in cardiac arrest patients treated with manual chest compressions compared with mechanical compressions with the LUCAS (TM) device-a pilot study. Resuscitation 2009;80(10):1104-7.

Stapleton 1991 \{published data only\}

Stapleton ER. Comparing CPR during ambulance transport. Manual vs. mechanical methods. Journal of Emergency Medical Services 1991;16(9):63-4.

\section{Stechovsky 2015 \{published data only\}}

Stechovsky C, Hajek P, Cipro S, Veselka J. Risk of myocardial contusion in cardiac arrest patients resuscitated with mechanical chest compression device. International Journal of Cardiology 2015;182:50-1.

\section{Swanson 2005a \{published data only\}}

Swanson M, Poniatowski M, O'Keefe M, Shedd JG. Effect of a CPR assist device on survival to emergency department arrival in out of hospital cardiac arrest. Circulation 2005;112(17):U1186.

\section{Swanson 2006a \{published data only\}}

Swanson M, Poniatowski M, O'Keefe M, Springer P. A CPR assist device increased emergency department admission and end tidal carbon dioxide partial pressures during treatment of out of hospital cardiac arrest. Circulation 2006;114(18):554.

\section{Swenson 1988a $\{$ published data only\}}

Swenson RD, Weaver WD, Niskanen RA, Martin J, Dahlberg S. Hemodynamics in humans during conventional and experimental methods of cardiopulmonary resuscitation. Circulation 1988;78(3):630-9.

Tucker 1993 \{published data only\}

Tucker KJ, Redberg RF, Schiller NB, Cohen TJ. Active compression-decompression resuscitation: analysis of transmitral flow and left ventricular volume by transesophageal echocardiography in humans. Cardiopulmonary Resuscitation Working Group. Journal of the American College of Cardiology 1993;22(5):1485-93.

\section{Tucker 1994 \{published data only\}}

Tucker KJ, Galli F, Savitt MA, Kahsai D, Bresnahan L, Redberg RF. Active compression-decompression resuscitation: effect on resuscitation success after in-hospital cardiac arrest. Journal of the American College of Cardiology 1994;24(1):201-9.

Vincent 2003 \{published data only\}

Vincent R. Resuscitation. Heart 2003;89(6):673-80.

\section{Wang 2007 \{published data only\}}

Wang HC, Chiang WC, Chen SY, Ke YL, Chi CL, Yang CW, et al. Video-recording and time-motion analyses of manual versus 
mechanical cardiopulmonary resuscitation during ambulance transport. Resuscitation 2007;74(3):453-60.

\section{Ward 1993 \{published data only\}}

Ward KR, Menegazzi JJ, Zelenak RR, Sullivan RJ, McSwain NE Jr. A comparison of chest compressions between mechanical and manual CPR by monitoring end-tidal PCO2 during human cardiac arrest. Annals of Emergency Medicine 1993;22(4):669-74.

\section{Weil 2000 \{published data only\}}

Weil MH. A new phased chest and abdominal compressiondecompression cardiopulmonary device holds promise for use in humans. Critical Care Medicine 2000;28(4):1240-1.

\section{Wik 2000 \{published data only\}}

Wik L. Automatic and manual mechanical external chest compression devices for cardiopulmonary resuscitation. Resuscitation 2000;47(1):7-25.

\section{Wolcke 2003 \{published data only\}}

Wolcke BB, Mauer DK, Schoefmann MF, Teichmann H, Provo TA, Lindner $\mathrm{KH}$, et al. Comparison of standard cardiopulmonary resuscitation versus the combination of active compressiondecompression cardiopulmonary resuscitation and an inspiratory impedance threshold device for out-of-hospital cardiac arrest. Circulation 2003;108(18):2201-5.

\section{Zoll 1966 \{published data only\}}

Zoll PM, Linenthal AJ. Control of heart action by electrical and mechanical means. Disease-A-Month 1966;Sep:2-25.

\section{References to ongoing studies}

\section{CTRI/2013/07/003840 \{unpublished data only\}}

CTRI/2013/07/003840. Randomised comparison of chest compression using the device AutoPulse ${ }^{\circledR}$ with manual chest compressions in patients requiring Cardiopulmonary Resuscitation (CPR) for in hospital cardiac arrest. http://ctri.nic.in/Clinicaltrials/pdf_generate.php? trialid=6068\&EncHid=\&modid=\&compid=\%27,\%276068det $\% 27$ (first received 25 July 2013).

\section{ISRCTN38139840 \{unpublished data only\}}

ISRCTN38139840. Mechanical and manual chest compressions for resuscitation in in-hospital cardiac arrest. https:// doi.org/10.1186/ISRCTN38139840 (first received 1 September 2017).

\section{ISRCTN78354073 \{unpublished data only\}}

ISRCTN78354073. German automatic chest compression resuscitation trial. https://doi.org/10.1186/ISRCTN78354073 (first received 1 July 2008).

\section{NCT01521208 \{published data only\}}

NCT01521208. LUCAS continuous chest compressions in out-ofhospital cardiac arrest treatment. The LUCAT trial [LUCAS chest compressor versus manual chest compression in out-of-hospital sudden cardiac arrest. LUCAT trial (LUCAT)]. clinicaltrials.gov/ ct2/show/record/NCT01521208 (first received 30 January 2012).

\section{Additional references}

\section{Abella 2005a}

Abella BS, Alvarado JP, Myklebust H, Edelson DP, Barry A, O'Hearn N, et al. Quality of cardiopulmonary resuscitation during in-hospital cardiac arrest. JAMA 2005;293(3):305-10.

\section{Abella 2005b}

Abella BS, Sandbo N, Vassilatos P, Alvarado JP, O'Hearn N, Wigder $\mathrm{HN}$, et al. Chest compression rates during cardiopulmonary resuscitation are suboptimal: a prospective study during in-hospital cardiac arrest. Circulation 2005;111(4):428-34.

\section{Babbs 1983}

Babbs CF, Voorhees WD, Fitzgerald KR, Holmes HR, Geddes LA. Relationship of blood pressure and flow during CPR to chest compression amplitude: evidence for an effective compression threshold. Annals of Emergency Medicine 1983;12(9):527-32. [0196-0644]

\section{Bellamy 1984}

Bellamy RF, DeGuzman LR, Pedersen DC. Coronary blood flow during cardiopulmonary resuscitation in swine. Circulation 1984;69(1):174-80. [0009-7322]

\section{Berg 2010}

Berg RA, Hemphill R, Abella BS, Aufderheide TP, Cave DM, Hazinski MF, et al. Part 5: adult basic life support 2010: American Heart Association Guidelines for Cardiopulmonary Resuscitation and Emergency Cardiovascular Care. Circulation 2010;122:S685-705.

\section{Bonnes 2016}

Bonnes JL, Brouwer MA, Navarese EP, Verhaert DV, Verheugt FW, Smeets JL, et al. Manual cardiopulmonary resuscitation versus CPR including a mechanical chest compression device in outof-hospital cardiac arrest: a comprehensive meta-analysis from randomized and observational studies. Annals of Emergency Medicine 2016;67(3):349-60.e3.

\section{Callaway 2015}

Callaway CW, Soar J, Aibiki M, Bottiger BW, Brooks SC, Deakin CD, et al. Part 4: advanced life support: 2015 International Consensus on Cardiopulmonary Resuscitation and Emergency Cardiovascular Care Science with Treatment Recommendations. Circulation 2015;132(16 Suppl 1):S84-145.

\section{Casner 2005}

Casner M, Andersen D, Isaacs SM. The impact of a new CPR device on rate of spontaneous circulation in out-of-hospital cardiac arrest. Prehospital Emergency Care 2005;9(1):61-7.

\section{de Latorre 2001}

de Latorre F, Nolan J, Robertson C, Chamberlain D, Baskett P, European Resuscitation Council. European Resuscitation Council Guidelines 2000 for Adult Advanced Life Support. A statement from the Advanced Life Support Working Group(1) and approved by the Executive Committee of the European Resuscitation Council. Resuscitation 2001;48(3):211-21. 


\section{Eftestol 2002}

Eftestol T, Sunde K, Steen PA. Effects of interrupting precordial compressions on the calculated probability of defibrillation success during out-of-hospital cardiac arrest. Circulation 2002;105(19):2270-3.

\section{Ewy 2005}

Ewy GA. Cardiocerebral resuscitation: the new cardiopulmonary resuscitation. Circulation 2005;111(16):2134-42. [0009-7322]

\section{Gates 2015}

Gates S, Quinn T, Deakin CD, Blair L, Couper K, Perkins GD. Mechanical chest compression for out of hospital cardiac arrest: Systematic review and meta-analysis. Resuscitation 2015;94:91-7.

\section{GRADEpro GDT 2015 [Computer program]}

McMaster University (developed by Evidence Prime). GRADEpro GDT. Version accessed 1 June 2018. Hamilton (ON): McMaster University (developed by Evidence Prime), 2015.

\section{Halperin 2002}

Halperin H, Paradis N, Ornato JP. Improved hemodynamics with a novel chest compression device during a porcine model of cardiac arrest. Circulation 2002;106:538.

\section{Halperin 2004}

Halperin HR, Paradis N, Ornato JP, Zviman M, LaCorte J, Lardo A, et al. Cardiopulmonary resuscitation with a novel chest compression device during a porcine model of cardiac arrest. Journal of the American College of Cardiology 2004;44(11):2214-20.

\section{Hasselqvist-Ax 2017}

Hasselqvist-Ax I, Riva G, Herlitz J, Rosenqvist M, Hollenberg J, Nordberg P, et al. Early cardiopulmonary resuscitation in outof-hospital cardiac arrest. New England Journal of Medicine 2015;372:2307-15.

\section{Heart and Stroke Foundation 2017}

Heart and Stroke Foundation of Canada. What is Cardiac Arrest?. www.heartandstroke.ca/heart/conditions/cardiacarrest (accessed 13 January 2017).

\section{Herlitz 1994}

Herlitz J, Ekstrom L, Wennerblom B, Axelsson A, Bang A Holmberg $\mathrm{S}$. Effect of bystander initiated cardiopulmonary resuscitation on ventricular fibrillation and survival after witnessed cardiac arrest outside hospital. British Heart Journal 1994;72(5):408-12.

\section{Herlitz 2000}

Herlitz J, Andersson E, Bang A, Engdahl J, Holmberg M, Lindqvist J, et al. Experiences from treatment of out-of-hospital cardiac arrest during 17 years in Goteborg. European Heart Journal 2000;21:1251-8.

\section{Higgins 2006}

Higgins JP, Green S, editor(s). Cochrane Handbook for Systematic Reviews of Interventions. Chichester (UK): John Wiley \& Sons, 2006.

\section{Higgins 2011}

Higgins JP, Green S, editor(s). Cochrane Handbook for Systematic Reviews of Interventions Version 5.1.0 (updated March 2011). The Cochrane Collaboration, 2011. Available from handbook.cochrane.org.

\section{Hightower 1995}

Hightower D, Thomas SH, Stone CK, Dunn K, March JA. Decay in quality of closed-chest compressions over time. Annals of Emergency Medicine 1995;26(3):300-3. [0196-0644]

\section{Idris 2012}

Idris AH, Guffey D, Aufderheide TP, Brown S, Morrison LJ, Nichols P, et al. Resuscitation Outcomes Consortium (ROC) Investigators. Relationship between chest compression rates and outcomes from cardiac arrest. Circulation 2012;125(24):3004-12.

\section{Idris 2015}

Idris AH, Guffey D, Pepe PE, Brown SP, Brooks SC, Callaway CW, et al. Chest compression rates and survival following out-ofhospital cardiac arrest. Critical Care Medicine 2015;43(4):840-8.

\section{Ikeno 2006}

Ikeno F, Kaneda H, Hongo Y, Sakanoue Y, Nolasco C, Emami S, et al. Augmentation of tissue perfusion by a novel compression device increases neurologically intact survival in a porcine model of prolonged cardiac arrest. Resuscitation 2006;68(1):109-18. [0300-9572]

\section{Jennett 1975}

Jennett B, Bond M. Assessment of outcome after severe brain damage: a practical scale. Lancet 1975;1:480-4.

\section{Kern 1986}

Kern K, Carter A, Showen R, Voorhees W, Babbs C, Tacker W, et al. Twenty-four hour survival in a canine model of cardiac arrest comparing three methods of manual cardiopulmonary resuscitation. Journal of the American College of Cardiology 1986;7:859-67.

\section{Kern 2002}

Kern K, Hilwig R, Berg R, Sanders A, Ewy G. Importance of continuous chest compressions during cardiopulmonary resuscitation: improved outcome during a simulated single layrescuer scenario. Circulation 2002;105(5):645-9. [0009-7322]

\section{Kleinman 2015}

Kleinman ME, Brennan EE, Goldberger ZD, Swor RA, Terry M, Bobrow BJ, et al. Part 5: Adult Basic Life Support and Cardiopulmonary Resuscitation Quality 2015 American Heart Association Guidelines Update for Cardiopulmonary Resuscitation and Emergency Cardiovascular Care. Circulation 2015;132:S414-35

\section{Ko 2005}

Ko PC, Chen WJ, Lin CH, Ma MH, Lin FY. Evaluating the quality of prehospital cardiopulmonary resuscitation by reviewing automated external defibrillator records and survival for outof-hospital witnessed arrests. Resuscitation 2005;64(2):163-9. [0300-9572] 


\section{Koster 2010}

Sayre MR, Koster RW, Botha M, Cave DM, Cudnik MT, Handley AJ, et al. Part 5: Adult basic life support: 2010 International Consensus on Cardiopulmonary Resuscitation and Emergency Cardiovascular Care Science with Treatment Recommendations. Resuscitation 2010;81:e48-70.

\section{Lefebvre 2011}

Lefebvre C, Manheimer E, Glanville J. Chapter 6: Searching for studies. In: Higgins JP, Green S, editor(s). Cochrane Handbook for Systematic Reviews of Interventions Version 5.1.0 (updated March 2011). The Cochrane Collaboration, 2011. Available from handbook.cochrane.org. Cochrane Collaboration.

\section{Lewis 2006b}

Lewis RJ, Niemann JT. Manual vs device-assisted CPR: reconciling apparently contradictory results. JAMA 2006;295(22):2661-4.

\section{Li 2016}

Li H, Wang D, Yu Y, Zhao X, Jing X. Mechanical versus manual chest compressions for cardiac arrest: a systematic review and meta-analysis. Scandinavian Journal of Trauma, Resuscitation and Emergency Medicine 2016;24:10.

\section{Maier 1984}

Maier G, Tyson G, Olsen C, Kernstein K, Davis J, Conn E, et al. The physiology of external cardiac massage: high-impulse cardiopulmonary resuscitation. Circulation 1984;70:86-101.

\section{Moher 2009}

Moher D, Liberati A, Tetzlaff J, Altman DG. Preferred reporting items for systematic reviews and meta-analyses: the PRISMA statement. PLoS Medicine 2009;6(7):e1000097.

\section{Monsieurs 2012}

Monsieurs KG, De Regge M, Vansteelandt K, De Smet J, Annaert $\mathrm{E}$, Lemoyne $\mathrm{S}$, et al. Excessive chest compression rate is associated with insufficient compression depth in prehospital cardiac arrest. Resuscitation 2012;83(11):1319-23.

\section{Mozaffarian 2016}

Mozaffarian D, Benjamin EJ, Go AS, Arnett DK, Blaha MJ, Cushman M, et al. American Heart Association Statistics Committee, Stroke Statistics Subcommittee. Heart Disease and Stroke Statistics-2016 Update: A Report From the American Heart Association. Circulation 2016;133(4):e38-360.

\section{Naim 2017}

Naim MY, Burke RV, McNally BF, Song L, Griffis HM, Berg RA, et al. Association of bystander cardiopulmonary resuscitation with overall and neurologically favorable survival after pediatric outof-hospital cardiac arrest in the United States: a report from the cardiac arrest registry to enhance survival surveillance registry. JAMA Pediatrics 2017;171(2):133-41.

\section{Nolan 2005}

Nolan JP, Deakin CD, Soar J, Bottiger BW, Smith G, European Resuscitation Council. European Resuscitation Council guidelines for resuscitation 2005. Section 4. Adult advanced life support. Resuscitation 2005;67 Suppl 1:S39-86.

\section{Ochoa 1998}

Ochoa FJ, Ramalle-Gomara E, Lisa V, Saralegui I. The effect of rescuer fatigue on the quality of chest compressions. Resuscitation 1998;37(3):149-52. [0300-9572]

\section{Ong 2006}

Ong ME, Ornato JP, Edwards DP, Dhindsa HS, Best AM, Ines CS, et al. Use of an automated, load-distributing band chest compression device for out-of-hospital cardiac arrest resuscitation. JAMA 2006;295(22):2629-37.

\section{Paradis 2010}

Paradis NA, Young G, Lemeshow S, Brewer JE, Halperin HR. Inhomogeneity and temporal effects in ASPIRE-an exception from consent trial terminated early. American Journal of Emergency Medicine 2010; Vol. 28:391-8.

\section{Perkins 2010a}

Perkins GD, Woollard M, Cooke MW, Deakin C, Horton J, Lall R, et al. Prehospital randomised assessment of a mechanical compression device in cardiac arrest (PaRAMeDIC) trial protocol. Scandinavian Journal of Trauma, Resuscitation and Emergency Medicine 2010;18:58.

\section{Perkins 2015a}

Perkins GD, Travers AH, Berg RA, Castren M, Considine J, Escalante R, et al. Part 3: Adult basic life support and automated external defibrillation: 2015 International Consensus on Cardiopulmonary Resuscitation and Emergency Cardiovascular Care Science with Treatment Recommendations. Circulation 2015;95:e43-69.

\section{Pusswald 2000}

Pusswald G, Fertl E, Faltl M, Auff E. Neurological rehabilitation of severely disabled cardiac arrest survivors: Part II. Life situation of patients and families after treatment. Resuscitation 2000;47:241-8.

\section{Review Manager 2014 [Computer program]}

Nordic Cochrane Centre, The Cochrane Collaboration. Review Manager 5 (RevMan 5). Version 5.3. Copenhagen: Nordic Cochrane Centre, The Cochrane Collaboration, 2014.

\section{Rubertsson 2005}

Rubertsson S, Karlsten R. Increased cortical cerebral blood flow with LUCAS; a new device for mechanical chest compressions compared to standard external compressions during experimental cardiopulmonary resuscitation. Resuscitation 2005;65:357-63.

\section{Ryan 2016}

Ryan R, Hill S. How to GRADE the quality of evidence. Cochrane Consumers and Communications Group December 2016; Vol. cccrg.cochrane.org/author-resources. [Version 3.0]

\section{Steen 2002}

Steen S, Liao Q, Pierre L, Paskevicius A, Sjoberg T, Steen S, et al. Evaluation of LUCAS, a new device for automatic mechanical compression and active decompression resuscitation. Resuscitation 2002;55(3):285-99. [0300-9572] 


\section{Steen 2005}

Steen S, Sjoberg T, Olsson P, Young M. Treatment of outof-hospital cardiac arrest with LUCAS, a new device for automatic mechanical compression and active decompression resuscitation. Resuscitation 2005;67(1):25-30. [0300-9572]

\section{Stiell 2004}

Stiell IG, Wells GA, Field B, Spaite DW, Nesbitt LP, De Maio VJ, et al. Advanced cardiac life support in out-of-hospital cardiac arrest. New England Journal of Medicine 2004;351(7):647-56.

\section{Stiell 2012}

Stiell IG, Brown SP, Christenson J, Cheskes S, Nichol G, Powell J, et al. Resuscitation Outcomes Consortium (ROC) Investigators. What is the role of chest compression depth during out-ofhospital cardiac arrest resuscitation?. Critical Care Medicine 2012;40(4):1192-8.

\section{Swanson 2005}

Swanson M, Poniatowski M, O'Keefe M, Shedd JG. Effect of a CPR assist device on survival to emergency department arrival in out of hospital cardiac arrest. Circulation 2005;112(17):U1186.

\section{Swanson 2006b}

Swanson M, Poniatowski M, O'Keefe M, Springer P. A CPR assist device increased emergency department admission and end tidal carbon dioxide partial pressures during treatment of out of hospital cardiac arrest. Circulation 2006;114(18):554.

\section{Swart 1994}

Swart G, Mateer J, DeBehnke D, Jameson S, Osborn J. The effect of compression duration on hemodynamics during mechanical high-impulse CPR. Academic Emergency Medicine 1994;1:430-7.

\section{Swenson 1988b}

Swenson R, Weaver W, Niskanen R, Martin J, Dahlberg S. Hemodynamics in humans during conventional and experimental methods of cardiopulmonary resuscitation. Circulation 1988;78:630-9.

\section{Tang 2015}

Tang Lu, Gu Wan-Jie, Wang Fei. Mechanical versus manual chest compressions for out-of-hospital cardiac arrest: a meta-analysis of randomized controlled trials. Scientific Reports 2015;5:15635.

\section{Timmerman 2003}

Timmerman S, Cardoso L, Ramires J. Improved hemodynamics with a novel chest compression device during treatment of inhospital cardiac arrest. Prehospital Emergency Care 2003;7:162.

\section{Utstein 2015}

Perkins GD, Jacobs IG, Nadkarni VM, Berg RA, Bhanji F, Biarent D, the Utstein Collaborators. Cardiac arrest and cardiopulmonary resuscitation outcome reports: update of the Utstein resuscitation registry templates for out-of-hospital cardiac arrest. Circulation 2015;132:1286-1300.

\section{Westfall 2013}

Westfall M, Krantz S, Mullin C, Kaufman C. Mechanical versus manual chest compressions in out-of-hospital cardiac arrest: a meta-analysis. Critical Care Medicine 2013;41(7):1782-9.

\section{Wik 2005}

Wik L, Kramer-Johansen J, Myklebust H, Sorebo H, Svensson L, Fellows $\mathrm{B}$, et al. Quality of cardiopulmonary resuscitation during out-of-hospital cardiac arrest. JAMA 2005;293(3):299-304.

\section{Young 2009}

Young G. Neurologic prognosis after cardiac arrest. New England Journal of Medicine 2009;361:605-11.

\section{Yu 2002}

Yu T, Weil MH, Tang W, Sun S, Klouche K, Povoas H, et al. Adverse outcomes of interrupted precordial compression during automated defibrillation. Circulation 2002;106(3):368-72.

\section{References to other published versions of this review Brooks 2008}

Brooks SC, Bigham B, Morrison LJ. Mechanical chest compressions versus manual chest compressions for cardiac arrest. Cochrane Database of Systematic Reviews 2008, Issue 3. [DOI: 10.1002/14651858.CD007260]

\section{Brooks 2011}

Brooks SC, Bigham BL, Morrison LJ. Mechanical versus manual chest compressions for cardiac arrest. Cochrane Database of Systematic Reviews 2011, Issue 1. [DOI: 10.1002/14651858.CD007260.pub2]

\section{Brooks 2014}

Brooks SC, Hassan N, Bigham BL, Morrison LJ. Mechanical versus manual chest compressions for cardiac arrest. Cochrane Database of Systematic Reviews 2014, Issue 2. [DOI: 10.1002/14651858.CD007260.pub3]

* Indicates the major publication for the study

\section{CHARACTERISTICS OF STUDIES}

Characteristics of included studies [ordered by study ID]

Dickinson 1998

$\begin{array}{ll}\text { Methods } & \text { Quasi-randomised trial in the USA } \\ \text { Randomisation based on odd/even calendar days }\end{array}$


Dickinson 1998 (Continued)

$$
\mathrm{N}=17
$$

Mechanical mean age: not reported. Male \%: not reported

Initial rhythm VF: $2 / 7$ (28.5\%). PEA: $2 / 7$ (28.5\%). Asystole: 3/7 (42.9\%)

Hypothermia treatment post-arrest: not reported

Manual mean age: not reported. Male \%: not reported

Initial rhythm VF: 4/10 (40\%). PEA: 4/10 (40\%). Asystole: 2/10 (20\%)

Hypothermia treatment post-arrest: not reported

Exclusions: none reported

\begin{tabular}{ll}
\hline Interventions & $\begin{array}{l}\text { Mechanical: "Thumper" piston at } 80 \text { compressions/min. Compression/ventilation ratio: not reported } \\
\text { Manual: chest compressions by paramedic. Compression rate: audio prompt for } 80 / \text { min Compres- } \\
\text { sion/ventilation ratio: not reported }\end{array}$ \\
\hline Outcomes & - ROSC: mechanical treatment $1 / 7(14.2 \%) ;$ manual $0 / 10(0 \%)$ \\
& Survival to hospital admission: mechanical $1 / 7(14.2 \%)$, manual $0 / 10(0 \%)$ \\
\hline Notes & $\begin{array}{l}\text { Primary outcome of study was end-tidal carbon dioxide. Reporting of baseline characteristics limited to } \\
\text { initial rhythm }\end{array}$
\end{tabular}

\section{Risk of bias}

\begin{tabular}{|c|c|c|}
\hline Bias & Authors' judgement & Support for judgement \\
\hline $\begin{array}{l}\text { Random sequence genera- } \\
\text { tion (selection bias) }\end{array}$ & High risk & Quasi-randomisation based on odd/even days \\
\hline $\begin{array}{l}\text { Allocation concealment } \\
\text { (selection bias) }\end{array}$ & High risk & Quasi-randomisation did not allow for allocation concealment \\
\hline $\begin{array}{l}\text { Blinding of participants } \\
\text { and personnel (perfor- } \\
\text { mance bias) } \\
\text { All outcomes }\end{array}$ & High risk & Not possible, given the nature of the intervention \\
\hline $\begin{array}{l}\text { Blinding of outcome as- } \\
\text { sessment (detection bias) } \\
\text { All outcomes }\end{array}$ & Unclear risk & Not reported \\
\hline $\begin{array}{l}\text { Incomplete outcome data } \\
\text { (attrition bias) } \\
\text { All outcomes }\end{array}$ & Low risk & All participants entered into the study had follow-up data \\
\hline $\begin{array}{l}\text { Selective reporting (re- } \\
\text { porting bias) }\end{array}$ & Unclear risk & Not reported \\
\hline Other bias & Unclear risk & $\begin{array}{l}\text { Both groups of participants placed on Thumper back-board. Manual CPR (con- } \\
\text { trol) group had machine running but not applied to participant to allow hu- } \\
\text { man compressions to be delivered at the same rate. }\end{array}$ \\
\hline
\end{tabular}


Gao 2016

\begin{tabular}{ll}
\hline Methods & Single-centre cluster-RCT in China \\
Clusters were patients treated by a particular ambulance
\end{tabular}

$\begin{array}{ll}\text { Participants } & \text { Adults with out-of-hospital atraumatic cardiac arrest with resuscitation attempted by EMS who were } \\ \text { admitted to the Emergency Medical Centre of one hospital }\end{array}$

$\mathrm{N}=133$

Mechanical mean age: 62.6 (14.9). Male: 50/69 (72.5\%). VF: 9/69 (13.0\%)

Initial rhythm VF/VT: 9/69 (13\%). PEA: 31/69 (44.9\%). Asystole: 24/69 (34.8\%)

Hypothermia treatment post-arrest: not reported

Manual mean age: 64.2 (12.6). Male: 44/64 (68.8\%). VF: 8/64 (12.5\%)

Initial rhythm VF/VT: 8/64 (12.5\%). PEA: 20/64 (31.3\%). Asystole: 32/64 (50\%)

Hypothermia treatment post-arrest: not reported

Exclusions: pregnant, trauma, patients with advanced cancer, aged $<14$ or $>90$ years old

Mechanical: manual compressions were started while the AutoPulse was being prepared
$\frac{\text { Manual: resuscitation (including defibrillation, drug administration and compression rate) followed the }}{2010 \text { American Heart Association Guidelines (Berg 2010) }}$

\begin{tabular}{|c|c|}
\hline Outcomes & $\begin{array}{l}\text { - Survival to hospital discharge: mechanical } 13 / 69(18.8 \%) \text {; manual } 4 / 64(6.3 \%) \\
\text { - Sustained ROSC: mechanical } 31 / 69(44.9 \%) \text {; manual } 15 / 64(23.4 \%) \\
\text { - Survival to } 24 \text { hours: mechanical } 27 / 69(39.1 \%) \text {; manual } 14 / 64(21.9 \%) \\
\text { - Survival to hospital discharge: mechanical } 13 / 69(18.8 \%) \text {; manual } 4 / 64(6.3 \%) \\
\text { - Sternal or rib fracture: mechanical } 4 / 60(6.7 \%) \text {; manual 3/63 (4.8\%) }\end{array}$ \\
\hline Notes & $\begin{array}{l}\text { Both study arms were intubated with ventilation consisting of } 100 \% \text { oxygen. End-tidal carbon dioxide } \\
\text { was monitored and recorded. No CPR process or quality data were recorded. CPR according to } 2010 \\
\text { American Heart Association CPR guidelines. Concern over selection bias based on how ambulances } \\
\text { were dispatched to cardiac arrest. Extremely high survival to hospital rate reported in mechanical com- } \\
\text { pression group in comparison to other published studies. Although "neurological prognosis" in the } \\
\text { form of Cerebral Performance Category is reported, this outcome is reported for all patients with ROSC, } \\
\text { not in the subset who survived to hospital discharge. }\end{array}$ \\
\hline
\end{tabular}

\section{Risk of bias}

Bias Authors' judgement Support for judgement

Random sequence genera- Low risk tion (selection bias)

Allocation concealment High risk
(selection bias)

The 10 ambulances of the Emergency Medical Centre were numbered one through 10. A computer then generated five numbers corresponding to five ambulances which were then equipped with an AutoPulse. The ambulances were then dispatched out sequentially to each patient with OHCA.

There was no allocation concealment as the order of which ambulance will be going out next was known. It is possible that ambulances known to be equipped with the mechanical device were dispatched to OHCA cases viewed as having a better prognosis. There were no mention of efforts to track any subterfuge of the randomisation process.

Blinding of participants High risk Not possible given the nature of the intervention
and personnel (perfor-
mance bias)
All outcomes


Gao 2016 (Continued)

Blinding of outcome as- Unclear risk Not reported sessment (detection bias)

All outcomes

Incomplete outcome data Unclear risk Not reported
(attrition bias)
All outcomes

\begin{tabular}{lll}
\hline $\begin{array}{l}\text { Selective reporting (re- } \\
\text { porting bias) }\end{array}$ & Unclear risk & Not reported \\
\hline Other bias & Low risk & All patients were reported to have received only their designated treatment. \\
\hline
\end{tabular}

\section{Hallstrom 2006}

$\begin{array}{ll}\text { Methods } & \text { Multicentre cluster-randomised trial in the USA and Canada } \\ \text { Clusters were based on ambulance station or group of stations with cross-over occurring at intervals } \\ \text { ranging from four weeks to two months }\end{array}$

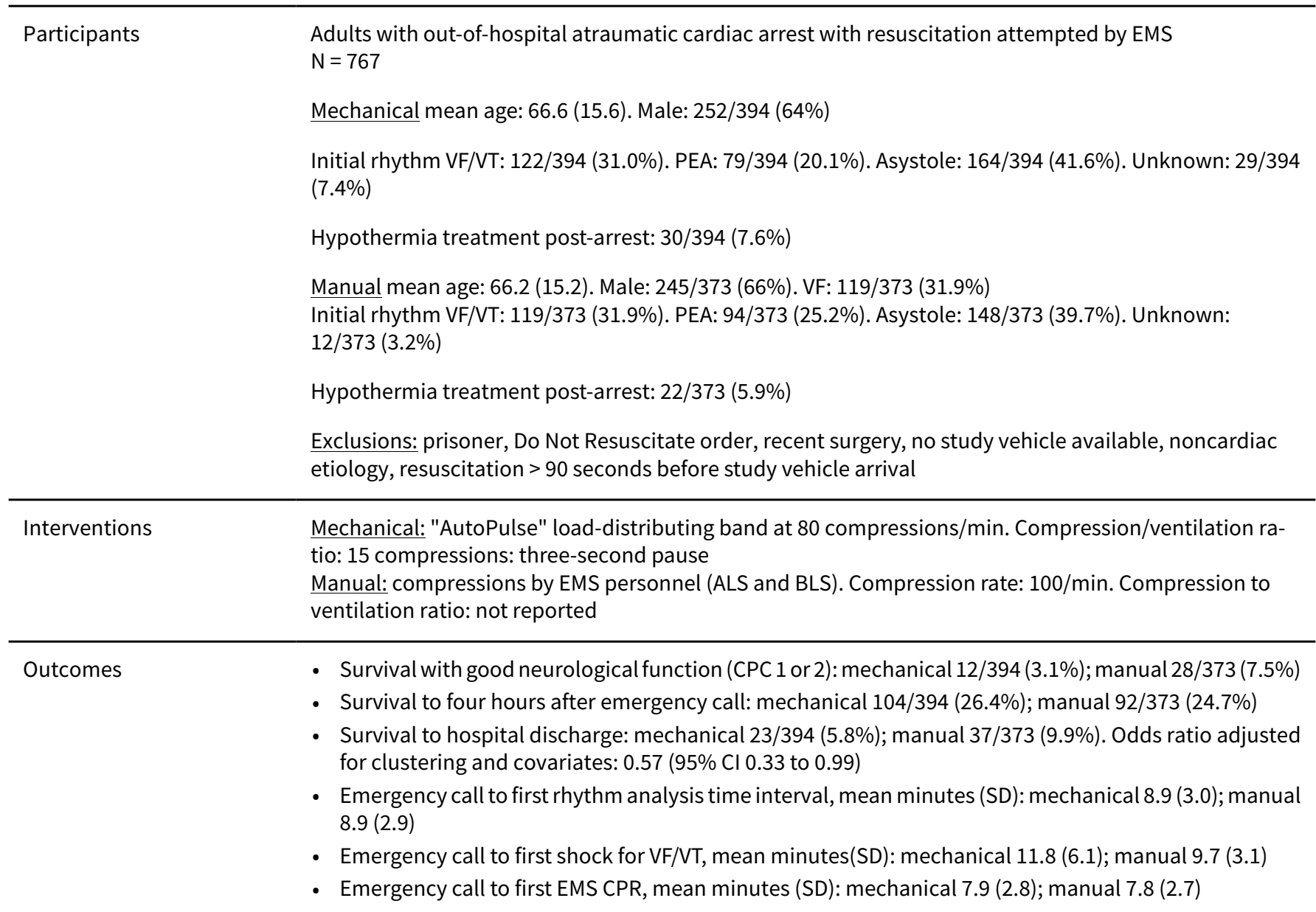

\section{Notes}
The study recruited participants in five cities, and the protocol for CPR was not uniform across all sites. In fact, the CPR protocol was changed part-way through the study at one site. The change involved a two-minute delay in applying the mechanical device to the participant, while paramedics administered manual CPR and a first defibrillation if needed. This change was incorporated in response to quality assurance data from the local emergency medical services system showing "prolonged time" without


Hallstrom 2006 (Continued)

compressions in the load-distributing band device group. The distribution of participant body type, as judged by treating paramedics, differed in the mechanical and manual groups. The mechanical group had more "thin" participants than the manual group (14.2\% versus $8.8 \%)$ and more "morbidly obese" participants than the manual group (4.3\% versus $2.4 \%)$. Trial stopped early at interim analysis for decreased survival to hospital discharge and no difference in primary outcome of survival to four hours after emergency call. Variable CPR protocols used across sites with respect to order of interventions and timing of device application. One of the larger study sites changed CPR protocol to delay application of device two minutes halfway through study

\section{Risk of bias}

\begin{tabular}{|c|c|c|}
\hline Bias & Authors' judgement & Support for judgement \\
\hline $\begin{array}{l}\text { Random sequence genera- } \\
\text { tion (selection bias) }\end{array}$ & Unclear risk & Not reported \\
\hline $\begin{array}{l}\text { Allocation concealment } \\
\text { (selection bias) }\end{array}$ & Unclear risk & Not reported \\
\hline $\begin{array}{l}\text { Blinding of participants } \\
\text { and personnel (perfor- } \\
\text { mance bias) } \\
\text { All outcomes }\end{array}$ & High risk & Not possible given the nature of the intervention \\
\hline $\begin{array}{l}\text { Blinding of outcome as- } \\
\text { sessment (detection bias) } \\
\text { All outcomes }\end{array}$ & Unclear risk & Not reported \\
\hline $\begin{array}{l}\text { Incomplete outcome data } \\
\text { (attrition bias) } \\
\text { All outcomes }\end{array}$ & Low risk & $99.3 \%$ of participants entered into the study had full follow-up data reported \\
\hline $\begin{array}{l}\text { Selective reporting (re- } \\
\text { porting bias) }\end{array}$ & Unclear risk & Not reported \\
\hline Other bias & High risk & $\begin{array}{l}\text { Site } \mathrm{C} \text { of the study changed the protocol halfway through the study; this in- } \\
\text { volved applying the device to participants after a period of CPR and rhythm } \\
\text { analysis. This change in CPR technique is likely to have had an impact on out- } \\
\text { comes for participants treated at this site with mechanical CPR. }\end{array}$ \\
\hline
\end{tabular}

\section{Halperin 1993}

\begin{tabular}{ll}
\hline Methods & Randomised controlled trial in the USA \\
\hline Participants & Patients with IHCA after unsuccessful standard CPR for less than 20 minutes \\
& $\mathrm{N}=34$ \\
& Mechanical mean age: 61 (16). Male: $10 / 17$ (58.8\%). VF: $3 / 17(17.6 \%)$ \\
& Initial rhythm VF/VT: 3/17 (17.6\%). PEA: 5/17 (29.4\%). Asystole: $6 / 17$ (35.3\%) \\
& Hypothermia treatment not reported \\
& Manual mean age: 69 (18). Male: $10 / 17$ (58.8\%). VF: $8 / 17(47.1 \%)$ \\
& Initial rhythm VF/VT: $8 / 17$ (47.1\%). PEA: $3 / 17(17.6 \%)$. Asystole: $3 / 17(17.6 \%)$ \\
& Hypothermia treatment not reported
\end{tabular}


Halperin 1993 (Continued)

$$
\text { Exclusions: patients given CPR for longer than } 20 \text { minutes before randomisation }
$$

\begin{tabular}{|c|c|}
\hline Interventions & $\begin{array}{l}\text { Mechanical: pneumatic vest. Compression rate: not reported. Compression/ventilation ratio: not re- } \\
\text { ported } \\
\text { Manual: chest compression provider type not reported. Compression rate: not reported Compres- } \\
\text { sion/ventilation ratio: not reported }\end{array}$ \\
\hline Outcomes & $\begin{array}{l}\text { - ROSC: mechanical } 8 / 17 \text { ( } 47.1 \%) \text {; manual } 3 / 17(17.6 \%) \\
\text { - Survival to six hours after resuscitation: mechanical } 6 / 17(35.3 \%) \text {; manual } 1 / 17(5.9 \%) \\
\text { - Survival to } 24 \text { hours after resuscitation: mechanical } 3 / 17(17.6 \%) \text {; manual } 1 / 17(5.9 \%) \\
\text { - Sternal or rib fracture: mechanical } 1 / 4(25 \%) \text {; manual } 2 / 5(40 \%) \\
\text { - Haemothorax or pneumothorax: mechanical 0/4 }(0 \%) \text {; manual } 1 / 5(20 \%)\end{array}$ \\
\hline Notes & $\begin{array}{l}\text { In-hospital study with late randomisation after "failed" standard CPR. Comorbidities, underlying cause } \\
\text { of cardiac arrest and important features of cardiac arrest circumstances (witness status, bystander CPR } \\
\text { before code team, etc.) not reported }\end{array}$ \\
\hline
\end{tabular}

\section{Risk of bias}

\begin{tabular}{|c|c|c|}
\hline Bias & Authors' judgement & Support for judgement \\
\hline $\begin{array}{l}\text { Random sequence genera- } \\
\text { tion (selection bias) }\end{array}$ & Unclear risk & Not reported \\
\hline $\begin{array}{l}\text { Allocation concealment } \\
\text { (selection bias) }\end{array}$ & Unclear risk & $\begin{array}{l}\text { Sequenced envelopes used. Unclear whether these envelopes were opaque; } \\
\text { no mention of a randomisation log to track any subterfuge of the randomisa- } \\
\text { tion process }\end{array}$ \\
\hline $\begin{array}{l}\text { Blinding of participants } \\
\text { and personnel (perfor- } \\
\text { mance bias) } \\
\text { All outcomes }\end{array}$ & High risk & Not practical given the nature of the intervention \\
\hline $\begin{array}{l}\text { Blinding of outcome as- } \\
\text { sessment (detection bias) } \\
\text { All outcomes }\end{array}$ & Unclear risk & Not reported \\
\hline $\begin{array}{l}\text { Incomplete outcome data } \\
\text { (attrition bias) } \\
\text { All outcomes }\end{array}$ & Unclear risk & Number of dropouts from the study not reported \\
\hline $\begin{array}{l}\text { Selective reporting (re- } \\
\text { porting bias) }\end{array}$ & Unclear risk & Not reported \\
\hline Other bias & Unclear risk & Randomisation in cases of unsuccessful manual CPR after cardiac arrest \\
\hline
\end{tabular}

Koster 2017

\begin{tabular}{ll}
\hline Methods & Non-inferiority RCT in the Netherlands \\
\hline Participants & IHCA or OHCA \\
& $N=374$
\end{tabular}


Mechanical AutoPulse mean age: 65 (15). Male: 75/115 (65.2\%). Initial rhythm: VF 30/115 (26\%), VT $7 / 115(6 \%)$, narrow complex tachycardia $2 / 115$ (2\%), PEA 42/115 (37\%), asystole 26/115 (23\%), unknown not shockable 5/115 (4\%). treatment post-arrest: not reported

LUCAS mean age: 63 (17). Male: 82/122 (67.2\%). Initial rhythm: VF 39/122 (32\%), VT 3/122 (2\%), narrow complex tachycardia 6/122 (6\%), PEA 36/122 (30\%), asystole 22/122 (18\%), unknown not shockable $10 / 122(8 \%)$. treatment post-arrest: not reported

Manual mean age: 87 (13.82). Male: 87/137 (63.5\%). VF 31/137 (23\%), VT 3/137 (2\%), narrow complex tachycardia 5/137 (4\%), PEA 49/137 (36\%), asystole 33/137 (24\%), unknown not shockable 9/137 (7\%). treatment post-arrest: not reported

Hypothermia treatment post-arrest: not reported

Exclusions: patients with traumatic cause of arrest, less than 18 years-old, patients with a mechanical chest compression device already applied prehospital by the ambulance crew, patients with ROSC prior to application of study device

Interventions Patients with either IHCA or OHCA with ongoing CPR upon arrival to hospital were randomised to either the AutoPulse or the LUCAS device. They were then randomised to either mechanical or manual compressions

Mechanical: mechanical chest compressions with either the LUCAS chest compression system or the AutoPulse device, which were operated by trained cardiac care unit nurses. Resuscitative protocol, compression rate and compression:ventilation ratio not reported

Manual: manual compressions with feedback from sternal displacement transducer from (Philips Heartstart MRx defibrillator). Resuscitative protocol, compression rate and compression:ventilation ratio not reported

Outcomes Primary outcome

- Serious or life-threatening visceral organ injury rate difference between mechanical device group and manual group. Autopulse rate difference $+5.3 \%(95 \% \mathrm{Cl}-2.2 \%$ to $12.8 \%), \mathrm{P}=0.15$. Rate difference LUCAS-control p1.0\% (95\% Cl - 5.5\% to $7.6 \%), \mathrm{P}=0.75$

\section{Secondary outcomes}

- Pneumothorax: mechanical AutoPulse 11/103 (10.6\%); mechanical LUCAS 3/108 (2.8\%); manual 4/126 $(3.2 \%)$

- Haemothorax: mechanical AutoPulse 2/103 (6.8\%); mechanical LUCAS 3/108 (2.8\%); manual 4/126 (3.2\%)

- Sternal or rib fractures: mechanical AutoPulse 47/103 (45.6\%); mechanical LUCAS 43/108 (39.8\%); manual 52/126 (41.3\%)

- Internal organ damage: mechanical AutoPulse 1/103 (<1\%); mechanical LUCAS 2/108 (< 1\%); manual $0 / 126(0 \%)$

Notes

This was a three-arm non-inferiority trial with resuscitation-related injuries as the primary outcome. Randomisation occurred before inclusion/exclusion criteria were applied. Baseline characteristics were balanced except for the exclusion criteria of ROSC on arrival of study resuscitation team, which the authors attribute to the lag time in applying the mechanical device, thus allowing more time for ROSC. Criteria for non-inferiority of LUCAS compared to manual chest compressions on the primary outcome of serious or life-threatening visceral organ injury ( $<10 \%$ difference) were satisfied. Criteria for non-inferiority of Autopulse compared to manual chest compressions on the primary outcome were not met

\section{Risk of bias}

Bias Authors' judgement Support for judgement

Random sequence genera- Low risk Computer-generated random numbers tion (selection bias) 
Koster 2017 (Continued)

\begin{tabular}{|c|c|c|}
\hline $\begin{array}{l}\text { Allocation concealment } \\
\text { (selection bias) }\end{array}$ & Low risk & $\begin{array}{l}\text { Used opaque sealed envelopes. No mention of a randomisation log to track } \\
\text { any subterfuge of the randomisation process or other safeguards against fore- } \\
\text { knowledge of sequential assignments }\end{array}$ \\
\hline
\end{tabular}

Blinding of participants High risk Not practical given the nature of the intervention
and personnel (perfor-
mance bias)
All outcomes

\begin{tabular}{|c|c|c|}
\hline $\begin{array}{l}\text { Blinding of outcome as- } \\
\text { sessment (detection bias) } \\
\text { All outcomes }\end{array}$ & Unclear risk & $\begin{array}{l}\text { The intention was for all assessors to be blinded to allocation. However, skin } \\
\text { markings from device were sometimes apparent to pathologists who were per- } \\
\text { forming the autopsies. }\end{array}$ \\
\hline
\end{tabular}

\begin{tabular}{lll}
\hline $\begin{array}{l}\text { Incomplete outcome data } \\
\text { (attrition bias) } \\
\text { All outcomes }\end{array}$ & Low risk & $\begin{array}{l}\text { For all outcomes, data were complete. Very few participants (1-2) had un- } \\
\text { known hospital course within each treatment arm. }\end{array}$ \\
\hline $\begin{array}{l}\text { Selective reporting (re- } \\
\text { porting bias) }\end{array}$ & Unclear risk & Not reported \\
\hline Other bias & Unclear risk & $\begin{array}{l}\text { Randomisation occurred prior to inclusion/exclusion criteria, which has an un- } \\
\text { clear impact on allocation. }\end{array}$ \\
\hline
\end{tabular}

Lu 2010

\begin{tabular}{|c|c|}
\hline Methods & RCT in China \\
\hline \multirow[t]{9}{*}{ Participants } & Confirmed IHCA confirmed by ECG or ECG monitor, cardiac arrest $\leq 10$ minutes \\
\hline & $N=150$ \\
\hline & Mechanical mean age: 47.72 (14.25). Male: $46 / 76(61 \%)$ \\
\hline & Initial rhythm: not reported \\
\hline & Hypothermia treatment post-arrest: not reported \\
\hline & Manual mean age: 45.50 (13.82). Male: $43 / 74(58 \%)$ \\
\hline & Initial rhythm: not reported \\
\hline & Hypothermia treatment post-arrest: not reported \\
\hline & $\begin{array}{l}\text { Exclusions: patients with extrathoracic and abdominal trauma, pregnant women, patients with termi- } \\
\text { nal illness and organ failure (e.g. terminal cancer, heart failure, multiple organ failure, etc.) }\end{array}$ \\
\hline \multirow[t]{2}{*}{ Interventions } & $\begin{array}{l}\text { Mechanical: Thumper Model } 1007 \mathrm{CCV} \text { at } 100 \text { compressions/min. Compression/ventilation ratio: five/ } \\
\text { one }\end{array}$ \\
\hline & Manual: compression/ventilation ratio: not reported \\
\hline \multirow[t]{2}{*}{ Outcomes } & - ROSC: mechanical $42 / 76$ (55.26\%); manual 28/74 (37.84\%) \\
\hline & - Survival to hospital discharge: mechanical 25/76 (32.89\%); manual 11/74 (14.86\%) \\
\hline Notes & $\begin{array}{l}\text { This study was published in Chinese. All information regarding design, results and risk of bias was ob- } \\
\text { tained with the help of interpreters }\end{array}$ \\
\hline
\end{tabular}

\section{Risk of bias}


Lu 2010 (Continued)

\begin{tabular}{lll} 
Bias & Authors' judgement & Support for judgement \\
\hline $\begin{array}{l}\text { Random sequence genera- } \\
\text { tion (selection bias) }\end{array}$ & Unclear risk & Method of randomisation not reported \\
\hline $\begin{array}{l}\text { Allocation concealment } \\
\text { (selection bias) }\end{array}$ & Unclear risk & Not reported \\
\hline $\begin{array}{l}\text { Blinding of participants } \\
\text { and personnel (perfor- } \\
\text { mance bias) } \\
\text { All outcomes }\end{array}$ & High risk & Not practical given the nature of the intervention \\
\hline
\end{tabular}

\begin{tabular}{|c|c|c|}
\hline $\begin{array}{l}\text { Blinding of outcome as- } \\
\text { sessment (detection bias) } \\
\text { All outcomes }\end{array}$ & Unclear risk & Not reported \\
\hline $\begin{array}{l}\text { Incomplete outcome data } \\
\text { (attrition bias) } \\
\text { All outcomes }\end{array}$ & Unclear risk & Not reported \\
\hline $\begin{array}{l}\text { Selective reporting (re- } \\
\text { porting bias) }\end{array}$ & Unclear risk & Not reported \\
\hline Other bias & Unclear risk & No evidence of this reported by translators. \\
\hline
\end{tabular}

Perkins 2015

$\begin{array}{ll}\text { Methods } & \text { Cluster-RCT in the UK } \\ \text { Clusters were patients treated by a particular ambulance }\end{array}$

Participants

Adult OHCA of presumed cardiac origin

$N=4471$

Mechanical mean age: 71.0 (16.3). Male: 1039/1652 (63\%)

Initial rhythm VF: 364/1652 (22\%). VT:12/1652 (1\%). PEA: 398/1652 (24\%). Asystole: 824/1652 (50\%). Unknown: 54/1652 (3\%)

Hypothermia treatment post-arrest: not reported

Manual mean age: 71.6 (16.1). Male: 1774/2819 (63\%)

Initial rhythm VF: 597/2819 (21\%). VT:18/2819 (1\%). PEA: 707/2819 (25\%). Asystole: 1384/2819 (49\%). Unknown: $113 / 2819$ (4\%)

Hypothermia treatment post-arrest: not reported

Exclusions: patients with traumatic cardiac arrest, known or clinically apparent pregnancy compressions between $40 \mathrm{~mm}$ to $53 \mathrm{~mm}$ at a rate of 102/min). Followed 2010 European Resuscitation Council Guidelines (Koster 2010)

Manual: compressions by EMS personnel (target compression depth of $50 \mathrm{~mm}$ to $60 \mathrm{~mm}$, rate 100 to 120/min). Followed 2010 European Resuscitation Council Guidelines (Koster 2010) 
Perkins 2015 (Continued)

Outcomes

- Survival to 30 days: mechanical 104/1652 (6\%); manual 193/2819 (7\%)

- ROSC: mechanical 522/1652 (32\%); manual 885/2819 (31\%)

- Survived event (ROSC sustained until admission and transfer of care to medical staff at the receiving hospital): mechanical 337/1652 (23\%); manual 658/2819 (23\%)

- Survival to 3 months: mechanical 96/1652 (6\%); manual 182/2819 (6\%)

- Survival to 12 months: mechanical 89/1652 (5\%); manual 175/2819 (6\%)

- Survival with favourable neurological function at 3 months (CPC score 1-2): mechanical 77/1652 (5\%); manual $168 / 2819(6 \%)$

\begin{tabular}{ll}
\hline Notes CPR quality was not monitored \\
\hline
\end{tabular}

\section{Risk of bias}

\begin{tabular}{|c|c|c|}
\hline Bias & Authors' judgement & Support for judgement \\
\hline $\begin{array}{l}\text { Random sequence genera- } \\
\text { tion (selection bias) }\end{array}$ & Low risk & $\begin{array}{l}\text { Ambulance vehicles were cluster-randomised with a computer generated se- } \\
\text { quence, stratified by station and vehicle type. }\end{array}$ \\
\hline $\begin{array}{l}\text { Allocation concealment } \\
\text { (selection bias) }\end{array}$ & Unclear risk & $\begin{array}{l}\text { Authors mention that ambulance dispatch staff were unaware of allocation. } \\
\text { No mention of a randomisation log to track any subterfuge of the randomisa- } \\
\text { tion process or other safeguards against foreknowledge of sequential assign- } \\
\text { ments. }\end{array}$ \\
\hline $\begin{array}{l}\text { Blinding of participants } \\
\text { and personnel (perfor- } \\
\text { mance bias) } \\
\text { All outcomes }\end{array}$ & High risk & Not practical given the nature of the intervention \\
\hline $\begin{array}{l}\text { Blinding of outcome as- } \\
\text { sessment (detection bias) } \\
\text { All outcomes }\end{array}$ & Low risk & Research nurses assessing patients at follow-up visits were blinded. \\
\hline $\begin{array}{l}\text { Incomplete outcome data } \\
\text { (attrition bias) } \\
\text { All outcomes }\end{array}$ & Low risk & $\begin{array}{l}\text { For the outcome of survival to } 30 \text { days, only } 1 \text { patient from the control arm was } \\
\text { lost to follow-up. For all other outcomes reported, incomplete data ranged } \\
\text { from } 0 \% \text { to } 6 \% \text { and was balanced in both arms. }\end{array}$ \\
\hline $\begin{array}{l}\text { Selective reporting (re- } \\
\text { porting bias) }\end{array}$ & Unclear risk & Not reported \\
\hline Other bias & High risk & $\begin{array}{l}\text { Unbalanced cross-over: mechanical to manual } 638 / 1652(39 \%) \text {; manual to me- } \\
\text { chanical } 11 / 2819(<1 \%)\end{array}$ \\
\hline
\end{tabular}

\section{Rubertsson 2014}

\begin{tabular}{ll}
\hline Methods & RCT in Sweden, the Netherlands, and the UK \\
\hline Participants & Adult OHCA of presumed cardiac origin \\
& $N=2589$ \\
& Mechanical mean age: 69.0 (range $16-100)$. Male: $869 / 1300$ (67\%) \\
& Initial rhythm VF/VT: $374 / 1300$ (29\%). PEA: $255 / 1300$ (20\%). Asystole: $610 / 1300$ (47\%). Unknown: \\
& 20/1300 (2\%) \\
& Hypothermia treatment post-arrest: $198 / 1300(15.2 \%)$
\end{tabular}


Rubertsson 2014 (Continued)

Manual mean age: 69.1 (range 15 to 99). Male: 857/1289 (66\%)

Initial rhythm VF/VT: 383/1289 (30\%). PEA: 254/1289 (20\%). Asystole: 594/1289 (46\%). Unknown: $16 / 1289(1 \%)$

Hypothermia treatment post-arrest: 214/1289 (16.6\%)

Exclusions: patients suffering traumatic cardiac arrest (including hanging), $<18$ years old, known pregnancy, too large or small to fit the device, undergoing defibrillation before the device arrived on scene, with crew-witnessed cardiac arrest who achieved ROSC after immediate defibrillation

Interventions

Mechanical: mechanical chest compressions with LUCAS chest compression system with non-standard treatment algorithm. Patients were treated with manual chest compressions while enrolment/randomisation occurred and if randomised to mechanical intervention, device was deployed immediately. Mechanical compressions were initiated and continued for 3-minute cycles. At 90 seconds into the cycle, defibrillation shock delivered without checking heart rhythm. Heart rhythm was checked after each 3-minute cycle. If a shockable rhythm was observed, a new 3-minute cycle was started; counter-shock delivered at 90 seconds without pausing; if no shockable rhythm was observed, a 3-minute cycle was initiated and continued without interruption.

Manual: compressions by EMS personnel according to 2005 European Resuscitation Council Guidelines (Nolan 2005)

Outcomes

- Survival to hospital discharge with good neurological function (CPC 1 or 2): mechanical 108/1300 (8.3\%); manual 100/1289 (7.8\%)

- Survival to hospital discharge: mechanical $117 / 1300$ (9.0\%); manual 118/1289 (9.2\%)

- Survival to hospital admission: mechanical 366/1300 (28.2\%); manual 357/1289 (27.7\%)

- Survival at 6 months: mechanical 111/1300 (8.5\%); manual 104/1289 (8.1\%)

- Survival to 4 hours: mechanical 307/1300 (23.6\%); manual 305/1289 (23.7\%)

- ROSC: mechanical 460/1300 (35.4\%); manual 446/1289 (34.6\%)

- Pneumothorax: mechanical $1 / 1300(<1 \%)$; manual $1 / 1289(<1 \%)$

- Sternal or rib fractures: mechanical $1 / 1300$ (<1\%; noted before application of device); manual 2/1289 $(<1 \%)$

- Internal organ damage: mechanical $1 / 1300(<1 \%$, suspected spleen rupture on computed tomagraph scan); manual 0/1289 (<1\%)

- Cardiac arrest to emergency call interval, median minutes (IQR): mechanical 2 (0-5); manual 2 (0-5)

- Cardiac arrest to start of manual CPR interval, median minutes (IQR): mechanical 11.5 (7-16); manual $11(7-15)$

- Cardiac arrest to start of mechanical CPR interval, median minutes (IQR): mechanical 15 (10-20); manual 18 (10-27)

- Cardiac arrest to first defibrillation interval, median minutes (IQR): mechanical 17 (12-22); manual 15.5 (11-23.5)

- Cardiac arrest to ROSC interval, median minutes (IQR): mechanical 17 (11-25); manual 14 (9-21)

Notes

Mechanical CPR arm followed a novel, study-specific algorithm (defibrillation without pulse/rhythm check and 3-min CPR periods) that may impact treatment effect. CPR quality monitored by impedence data in $10 \%$ of patients showing CPR fraction of 0.78 and 0.84 in the manual and mechanical groups, respectively

\section{Risk of bias}

\begin{tabular}{lll}
\hline Bias & Authors' judgement & Support for judgement \\
\hline $\begin{array}{l}\text { Random sequence genera- } \\
\text { tion (selection bias) }\end{array}$ & Unclear risk & $\begin{array}{l}\text { Refers to sealed randomisation envelopes. No other information, including } \\
\text { method of randomisation, provided }\end{array}$ \\
\hline
\end{tabular}


Rubertsson 2014 (Continued)

Allocation concealment Low risk Used opaque sealed envelopes. No mention of a randomisation log to track (selection bias)

\section{any subterfuge of the randomisation process or other safeguards against fore-} knowledge of sequential assignments

Blinding of participants High risk Not practical given the nature of the intervention
and personnel (perfor-

$\begin{array}{ll}\text { Blinding of outcome as- } & \text { Unclear risk }\end{array}$

All outcomes

\begin{tabular}{|c|c|c|}
\hline $\begin{array}{l}\text { Incomplete outcome data } \\
\text { (attrition bias) }\end{array}$ & Low risk & $\begin{array}{l}\text { For all outcomes, incomplete data occurred in } 1 \% \text { or less of cases. Missing } \\
\text { data were imputed as the "worst case" in the intention-to-treat population }\end{array}$ \\
\hline
\end{tabular}

All outcomes

data were imputed as the "worst case" in the intention-to-treat population

\begin{tabular}{lll}
\hline $\begin{array}{l}\text { Selective reporting (re- } \\
\text { porting bias) }\end{array}$ & Unclear risk & Not reported \\
\hline Other bias & Unclear risk & $\begin{array}{l}\text { Cross-over contamination from mechanical to manual arm and vice versa, } \\
64 / 1300(4.9 \%) \text { and } 46 / 1293(3.6 \%) \text { respectively. Cointervention present in me- } \\
\text { chanical CPR arm (study-specific ALS treatment algorithm) }\end{array}$ \\
\hline
\end{tabular}

Smekal 2011

\begin{tabular}{ll}
\hline Methods & RCT in Sweden \\
\hline Participants & Out-of-hospital sudden cardiac arrest \\
$N=148$ & Mechanical mean age: 69 (16). Male \%: 50/75 (68\%) \\
Initial rhythm VF/VT: VF: 20/75 (27\%) \\
Hypothermia treatment post-arrest: not reported \\
Manual mean age: 71 (16). Male \%: $50 / 73$ (68\%) \\
Initial rhythm VF/VT: $20 / 73$ (27\%) \\
Hypothermia treatment post-arrest: not reported \\
Exclusions: known pregnancy, younger than 18 years of age or trauma
\end{tabular}

Interventions

\begin{abstract}
Mechanical: "LUCAS" piston with active compression-decompression CPR. Participants in the mechanical chest compression group received manual chest compressions while the device was being prepared. They then received 90 seconds of LUCAS compression. The following cycle was then repeated twice: Check rhythm and pulse for a maximum of 10 seconds; if shockable, provide LUCAS for 90 seconds while fixing intravenous line, shock once (200J), provide LUCAS for 60 seconds and repeat. If the participant's rhythm was non-shockable, LUCAS compressions were continued for 90 seconds and repeated. After two cycles of this modified algorithm, traditional CPR, according to European Resuscitation Council 2000 guidelines for advanced cardiac life support, was provided with the use of LUCAS to deliver chest compressions
\end{abstract}

Manual: chest compressions by nurse. Compression rate: performed according to European Resuscitation Council 2000 guidelines (de Latorre 2001), not otherwise reported. Compression/ventilation ratio: 
Smekal 2011 (Continued)

performed according to European Resuscitation Council 2000 guidelines (de Latorre 2001), not otherwise reported

\begin{tabular}{ll}
\hline Outcomes & ROSC: mechanical 30/74 (41\%); manual 23/72 (32\%) \\
& - Survival to hospital discharge: mechanical 6/75 (8\%); manual 7/72 (10\%) \\
& - Survival to hospital admission: mechanical $18 / 75(24 \%) ;$ manual $15 / 72(21 \%)$ \\
& Scene arrival to first CPR time interval mean minutes (SD): mechanical $1.0(1.1) ;$ manual $1.1(1.1)$ \\
\hline Notes & $\begin{array}{l}\text { Primary outcome of the study was ROSC with BP > 80/50 mmHg for > five minutes. LUCAS algorithm dif- } \\
\text { fered from manual CPR algorithm for the first two cycles }\end{array}$ \\
\hline
\end{tabular}

\section{Risk of bias}

\begin{tabular}{|c|c|c|}
\hline Bias & Authors' judgement & Support for judgement \\
\hline $\begin{array}{l}\text { Random sequence genera- } \\
\text { tion (selection bias) }\end{array}$ & Unclear risk & Not reported \\
\hline $\begin{array}{l}\text { Allocation concealment } \\
\text { (selection bias) }\end{array}$ & Unclear risk & $\begin{array}{l}\text { Sequenced envelopes used. Unclear whether these envelopes were opaque; } \\
\text { no mention of a randomisation log to track any subterfuge of the randomisa- } \\
\text { tion process }\end{array}$ \\
\hline $\begin{array}{l}\text { Blinding of participants } \\
\text { and personnel (perfor- } \\
\text { mance bias) } \\
\text { All outcomes }\end{array}$ & High risk & Not practical given the nature of the intervention \\
\hline $\begin{array}{l}\text { Blinding of outcome as- } \\
\text { sessment (detection bias) } \\
\text { All outcomes }\end{array}$ & Unclear risk & Not reported \\
\hline $\begin{array}{l}\text { Incomplete outcome data } \\
\text { (attrition bias) } \\
\text { All outcomes }\end{array}$ & Low risk & $\begin{array}{l}\text { Following enrolment, only one participant was excluded from the study be- } \\
\text { cause he/she was not randomly assigned correctly. For all survival study out- } \\
\text { comes, no more than one participant per group had missing data }\end{array}$ \\
\hline $\begin{array}{l}\text { Selective reporting (re- } \\
\text { porting bias) }\end{array}$ & Unclear risk & Not reported \\
\hline Other bias & High risk & LUCAS algorithm differed from manual CPR algorithm for the first two cycles \\
\hline
\end{tabular}

Taylor 1978

\begin{tabular}{ll}
\hline Methods & RCT \\
\hline Participants & Patients with IHCA undergoing "prolonged" CPR \\
$\mathrm{N}=50$ \\
Mechanical mean age: 57 (5.2). Male: not reported \\
Initial rhythm: not reported \\
Hypothermia treatment post-arrest: not reported \\
Manual mean age: 54.8 (6.6). Male: not reported \\
Initial rhythm: not reported
\end{tabular}


Taylor 1978 (Continued)

Hypothermia treatment post-arrest: not reported

Exclusions: patients with greater than 10 minutes of standard CPR

$\begin{array}{ll}\text { Interventions } & \text { Mechanical: piston at } 60 \text { compressions/min. Compression/ventilation ratio: five/one } \\ & \text { Manual: chest compressions by medical house staff. Compression rate: not reported Compression/ven- } \\ \text { tilation ratio: not reported }\end{array}$

tilation ratio: not reported

\begin{tabular}{ll}
\hline Outcomes & Survival to hospital discharge: mechanical 3/24 (12.5\%); manual 2/26 (7.7\%) \\
- Survival to 24 hours after CPR: mechanical 4/24 (16.7\%); manual 4/26 $(15.4 \%)$ \\
- Sternal or rib fractures: mechanical 10/13 (76.9\%); manual 8/17 (47.1\%) \\
- Internal organ damage: mechanical 0/13 (0\%); manual 2/17 (11.8\%) \\
\hline Notes & $\begin{array}{l}\text { Older in-hospital study. Lack of reporting of participant characteristics, reason for arrest, initial rhythm. } \\
\text { Potential selection bias in adverse effect risk estimates, as autopsies were not universal and indications } \\
\text { for autopsy were not reported }\end{array}$ \\
\hline
\end{tabular}

\section{Risk of bias}

\begin{tabular}{|c|c|c|}
\hline Bias & Authors' judgement & Support for judgement \\
\hline $\begin{array}{l}\text { Random sequence genera- } \\
\text { tion (selection bias) }\end{array}$ & Unclear risk & Method of randomisation is described as "drawing cards" \\
\hline $\begin{array}{l}\text { Allocation concealment } \\
\text { (selection bias) }\end{array}$ & Unclear risk & Not reported \\
\hline $\begin{array}{l}\text { Blinding of participants } \\
\text { and personnel (perfor- } \\
\text { mance bias) } \\
\text { All outcomes }\end{array}$ & High risk & Not practical given the nature of the intervention \\
\hline $\begin{array}{l}\text { Blinding of outcome as- } \\
\text { sessment (detection bias) } \\
\text { All outcomes }\end{array}$ & Unclear risk & $\begin{array}{l}\text { Not reported with respect to pathological examination for injuries caused by } \\
\text { the two different types of chest compressions }\end{array}$ \\
\hline $\begin{array}{l}\text { Incomplete outcome data } \\
\text { (attrition bias) } \\
\text { All outcomes }\end{array}$ & Low risk & $\begin{array}{l}\text { All participants who entered the study were accounted for with respect to out- } \\
\text { comes reported }\end{array}$ \\
\hline $\begin{array}{l}\text { Selective reporting (re- } \\
\text { porting bias) }\end{array}$ & Unclear risk & Not reported \\
\hline Other bias & Unclear risk & $\begin{array}{l}\text { Process of CPR in each of the groups was not reported. Unclear whether proto- } \\
\text { col related to administration of mechanical CPR; may have altered the process } \\
\text { of resuscitation to result in a cointervention }\end{array}$ \\
\hline
\end{tabular}

Wik 2014

\begin{tabular}{ll}
\hline Methods & RCT in the USA, Austria and the Netherlands \\
\hline Participants & Adult OHCA of presumed cardiac origin \\
$N=4231$ \\
Mechanical mean age: $65.7 \pm 16.4$. Male: $1295 / 2099(61 \%)$
\end{tabular}


Wik 2014 (Continued)

Initial rhythm VF/VT: 451/2099 (21\%). PEA/asystole: 1572/2099 (75\%). Unknown: 76/2099 (4\%)

Hypothermia treatment post-arrest: 690/2099 (32.9\%)

Manual mean age: $65.6 \pm 16.0$. Male: $1315 / 2132(61 \%)$

Initial rhythm VF/VT: 519/2132 (24\%). PEA/asystole: 1516/2132 (71\%). Unknown: 97/2132 (5\%)

Hypothermia treatment post-arrest: 800/2123 (37.5\%)

Exclusions: patients with Do Not Resuscitate orders, who had received mechanical chest compressions prior to randomisation, presumed pregnant, too large for CPR device, prisoners, or if the randomising EMS unit arrived more than 16 mins after emergency call

Interventions $\quad$ Mechanical: integrated AutoPulse CPR (iA-CPR). Upon arrival, one EMS personnel performed manual $\mathrm{CPR}$ and another opened a randomisation envelope. If randomised to receive iA-CPR, the AutoPulse compressions were immediately initiated

Manual: compressions by EMS personnel in accordance with the American Heart Association 2005 Guidelines (except resuscitation cycle was 3 mins)

\begin{tabular}{|c|c|}
\hline Outcomes & $\begin{array}{l}\text { - Survival to discharge with good neurological function (mRS 0-3): mechanical 87/2099 (4.1\%); manual } \\
112 / 2132(5.3 \%) \\
\text { - Survival to hospital discharge: mechanical } 196 / 2099(9.4 \%) \text {; manual } 233 / 2132(11.0 \%) \\
\text { - Sustained ROSC: mechanical 600/2099 (28.6\%); manual 689/2132 (32.3\%) } \\
\text { - Survival to } 24 \text { hours: mechanical } 456 / 2099(21.8 \%) \text {; manual 532/2132 }(25.0 \%) \\
\text { - Sternal or rib fracture: mechanical 70/2099 (3\%); manual 36/2131 (2\%) } \\
\text { - Haemothorax or pneumothorax: mechanical 34/2099 (2\%); } 21 / 2132(1 \%) \\
\text { - Abdominal organ injury: mechanical } 1 / 2099(<1 \%) ; 0 / 2132(0 \%) \\
\text { - Scene arrival to first shock time interval for VT/VF (mean } \pm \text { SD): mechanical } 7.5 \pm 6.0 \text { min; manual } 6.7 \\
\pm 6.2 \text { mins }\end{array}$ \\
\hline
\end{tabular}

Notes

This study design comprised of 3 phases: 1 ) in-field training where all OHCA patients were treated with AutoPulse device followed by 2 ) run-in phase to evaluate protocol compliance and minimise Hawthorne effect prior to 3) statistical inclusion phase.

Authors emphasised the rigorous training for EMS personnel and continuous monitoring of CPR quality, user compliance. $96 \%$ of enrolled cases were monitored for CPR fraction. Both the proportion monitored and the CPR fraction were found to be higher than in previous studies. May reduce generalisability to real-world CPR provider quality

\section{Risk of bias}

\begin{tabular}{lll}
\hline Bias & Authors' judgement & Support for judgement \\
\hline $\begin{array}{l}\text { Random sequence genera- } \\
\text { tion (selection bias) }\end{array}$ & Unclear risk & $\begin{array}{l}\text { Refers to sealed randomisation cards. No other information, including method } \\
\text { of randomisation, provided }\end{array}$ \\
\hline $\begin{array}{l}\text { Allocation concealment } \\
\text { (selection bias) }\end{array}$ & Unclear risk & $\begin{array}{l}\text { Sealed envelopes. Does not say if envelope was opaque. No mention of a ran- } \\
\text { domisation log to track any subterfuge of the randomisation process or other } \\
\text { safeguards against foreknowledge of sequential assignments }\end{array}$ \\
\hline $\begin{array}{l}\text { Blinding of participants } \\
\text { and personnel (perfor- } \\
\text { mance bias) }\end{array}$ & High risk & Not practical given the nature of the intervention \\
\hline $\begin{array}{l}\text { All outcomes } \\
\text { sessment (detection bias) }\end{array}$ & High risk & $\begin{array}{l}\text { Incomplete blinding as authors state study personnel were "not always blind- } \\
\text { ed to study arm" }\end{array}$
\end{tabular}


Wik 2014 (Continued)

All outcomes

Incomplete outcome data Low risk (attrition bias)

All outcomes
Primary outcome (survival to discharge) data available for 4219/4231 (99.1\%)

Neurological function (mRS scores) of patients who survived to discharge available only for $310 / 429$ (overall $72 \%$; mechanical $70 \%$; manual $74 \%$ ). Authors state that this was likely not related to their neurological condition, but rather the ability of research co-ordinator to promptly locate and obtain consent from patient prior to discharge. 262 (11\%) controls and $260(11 \%)$ iA-CPR participants were excluded post-enrolment

\begin{tabular}{lll}
\hline $\begin{array}{l}\text { Selective reporting (re- } \\
\text { porting bias) }\end{array}$ & Unclear risk & Not reported \\
\hline Other bias & Unclear risk & Not reported \\
\hline
\end{tabular}

Abbreviations: $\mathrm{ALS}=$ advanced life support; $\mathrm{BLS}=$ basic life support; $\mathrm{BP}=$ blood pressure; $\mathrm{CPC}=$ Cerebral Performance Score; $\mathrm{CPR}$ = cardiopulmonary resuscitation; ECG = electrocardiogram; EMS = emergency medical services; IHCA = in-hospital cardiac arrest; IQR = interquartile range; $\mathrm{mRS}=$ modified Rankin Scale; OHCA = out-of-hospital cardiac arrest; $\mathrm{PEA}=$ pulseless electrical activity; $\mathrm{RCT}=$ randomised controlled trial; ROSC = return of spontaneous circulation; VF = ventricular fibrillation; $\mathrm{VT}=$ ventricular tachycardia

\section{Characteristics of excluded studies [ordered by study ID]}

\begin{tabular}{|c|c|}
\hline Study & Reason for exclusion \\
\hline Anonymous 1973 & $\begin{array}{l}\text { Non-randomised study of technical specifications of several powered and manual devices. No data } \\
\text { from use on humans }\end{array}$ \\
\hline Arntz 1998 & Review \\
\hline Arntz 2001 & No mechanical CPR device; manual active compression-decompression device \\
\hline Aufderheide 2011 & Did not include the use of a mechanical chest compression device \\
\hline Axelsson 2006 & Non-randomised cluster trial; verified with author communication \\
\hline Axelsson 2009 & $\begin{array}{l}\text { Prospective observational study inappropriately called a "pseudo-randomised cluster study". } \\
\text { As stated in the methods, the clusters or individual patients were not actually randomised. The } \\
\text { methodology for allocation used does not fit the definition for quasi-randomisation. }\end{array}$ \\
\hline Baubin 1999 & No mechanical CPR device; manual active compression-decompression device \\
\hline Box 2008 & Observational study \\
\hline Dittbrenner 1993 & Review of computerised patient record, not cardiopulmonary resuscitation \\
\hline Dotter 1961 & Case series \\
\hline Elich 1995 & Review \\
\hline Halperin 2000 & Animal study \\
\hline Hampe 2008 & Case series \\
\hline Harkins 1961 & Review \\
\hline
\end{tabular}




\begin{tabular}{|c|c|}
\hline Study & Reason for exclusion \\
\hline Havel 2008 & No mechanical CPR device; manual active compression-decompression device \\
\hline Kern 2001 & Review \\
\hline Knight 1964 & Review \\
\hline Krischer 1989 & Included patients with drowning and toxicology as presumed causes of cardiac arrest \\
\hline Kurowski 2015 & No mechanical CPR device. CardioPump CPR feedback device \\
\hline Lairet 2005 & Retrospective chart review \\
\hline Liu 2010 & Study was not randomised \\
\hline Lurie 1994 & No mechanical CPR device; manual active compression-decompression device \\
\hline Lurie 1997 & Review \\
\hline Lurie 2002 & Review \\
\hline Malzer 1996 & No mechanical CPR device; manual active compression-decompression device \\
\hline Montgomery 1995 & Review \\
\hline Morozumi 2009 & Case report \\
\hline Nachlas 1962 & Review \\
\hline Nachlas 1963 & Animal study \\
\hline Nachlas 1965 & Review/case study \\
\hline Niemann 1984 & Abstract on cough CPR \\
\hline Nishino 1992 & $\begin{array}{l}\text { Studied anaesthetised patients not in cardiac arrest and the effect of static chest compression on } \\
\text { respiratory characteristics }\end{array}$ \\
\hline Pearson 1966 & Manikin study \\
\hline Plaisance 1999 & No mechanical CPR device; manual active compression-decompression device \\
\hline Rivers 1993 & Non-randomised study \\
\hline Roberts 1978 & Case series \\
\hline Schwab 1995 & No mechanical CPR device; manual active compression-decompression device \\
\hline Skogvoll 1999 & No mechanical CPR device; manual active compression-decompression device \\
\hline Smekal 2009 & Observational study \\
\hline Stapleton 1991 & Manikin study \\
\hline Stechovsky 2015 & Non-randomised study \\
\hline
\end{tabular}




\begin{tabular}{|c|c|}
\hline Study & Reason for exclusion \\
\hline Swanson 2005a & Non-randomised study \\
\hline Swanson 2006a & Non-randomised study \\
\hline Swenson 1988a & Case series \\
\hline Tucker 1993 & No mechanical CPR device; manual active compression-decompression device \\
\hline Tucker 1994 & No mechanical CPR device; manual active compression-decompression device \\
\hline Vincent 2003 & Review \\
\hline Wang 2007 & Non-randomised study looking at CPR quality, not clinical outcomes \\
\hline Ward 1993 & $\begin{array}{l}\text { Cross-over study after failed resuscitation. Subjects received both manual and mechanical chest } \\
\text { compressions with end-tidal } \mathrm{CO}^{2} \text { as outcome }\end{array}$ \\
\hline Weil 2000 & Review of manual adjunct device \\
\hline Wik 2000 & Review \\
\hline Wolcke 2003 & No mechanical CPR device; manual active compression-decompression device \\
\hline Zoll 1966 & Review \\
\hline
\end{tabular}

CPR = cardiopulmonary resuscitation

Characteristics of ongoing studies [ordered by study ID]

\section{CTRI/2013/07/003840}

Trial name or title Randomised comparison of chest compression using the device AutoPulse with manual chest compressions in patients requiring cardiopulmonary resuscitation (CPR) for in hospital cardiac arrest CAPCAR

\begin{tabular}{|c|c|}
\hline Methods & Randomised controlled trial \\
\hline Participants & $\begin{array}{l}\text { Inclusion criteria: patient with cardiac arrest in the emergency department. Exclusion criteria: }<18 \\
\text { years, chest trauma, patients already on advanced life support, patients achieving ROSC after the } \\
\text { initial } 5 \text { minutes of manual cardiopulmonary resuscitation according to the } 2010 \text { guidelines, pre- } \\
\text { dicted futile resuscitation based on fixed dilated pupils, Glascow Coma Scale } 3 / 15 \text {, absent pupillary } \\
\text { and other brain stem reflexes in the absence of drug intoxications, pregnant females, patients with } \\
\text { "Do Not Resuscitate" orders }\end{array}$ \\
\hline
\end{tabular}

Interventions Intervention: AutoPulse: mechanical chest compression device. It will provide chest compression at $100 /$ mins for the duration of resuscitation

Control intervention: manual CPR: CPR will be done utilising conventional manual chest compression with hands with targeted rate at least 100 /mins for the duration of resuscitation

\section{Outcomes}

\section{Primary efficacy endpoints}

- Sustained ROSC: admission to intense care unit/wards with a palpable pulse and measurable blood pressure

- Overall survival

- Survival to $24 \mathrm{hrs}$ : being alive $24 \mathrm{hrs}$ after the initial arrest 
Timepoints:

- Sustained ROSC at 24 hours

- Overall survival at hospital discharge

\section{Secondary efficacy endpoints}

- In patients with survival to discharge

* Modified Rankin Scale

* Cerebral Performance Category

- APACHE III Score

Timepoint:

- Survival at $24 \mathrm{hrs}$ and at discharge

\begin{tabular}{ll}
\hline Starting date & 01/06/2013 \\
\hline Contact information & Dr. Azharuddin Mohammed Malik \\
& Department of Medicine Jawaharlal Nehru Medical College, Aligarh Muslim University 202002 Ali- \\
garh, UTTAR PRADESH, India & Telephone: 8126320218 \\
e-mail: malikazharuddin@gmail.com
\end{tabular}

Notes

\section{ISRCTN38139840}

Trial name or title

A feasibility randomised controlled trial of mechanical chest compression devices for in-hospital cardiac arrest (COMPRESS-RCT)

\begin{tabular}{ll}
\hline Methods & Randomised \\
\hline Participants & Adults with in-hospital cardiac arrest \\
\hline Interventions & $\begin{array}{l}\text { Participants are 3:1 randomised to either receive mechanical or manual chest compressions. Me- } \\
\text { chanical compressions delivered by LUCAS-2 or LUCAS-3 device (Joliffe AB/ Physio-Control, Lund, } \\
\text { Sweden) }\end{array}$ \\
\hline
\end{tabular}

Outcomes

\section{Primary outcome}

- Proportion of eligible participants randomised over study period

\section{Secondary outcomes}

- Measures of feasibility

- ROSC

- Survival at discharge (with good neurological function)

- Survival at 30 days

- Survival at 6 months (with good neurological function)

- Hospital length of stay

- Quality of life measures at discharge and 6 months

- CPR quality 
ISRCTN38139840 (Continued)

- Adverse events profile

\begin{tabular}{ll}
\hline Starting date & October 2015 \\
\hline Contact information & Dr. Keith Couper; phone +442476575923 \\
\hline Notes & Patient recruitment officially began in late February 2017 per study website \\
\hline
\end{tabular}

\section{ISRCTN78354073}

\begin{tabular}{ll}
\hline Trial name or title & German Automatic chest compression Resuscitation Trial (German ART) \\
\hline Methods & Randomised \\
\hline Participants & Adults aged $18-80$ years old with non-traumatic out-of-hospital cardiac arrest \\
& $\begin{array}{l}\text { Exclusion criteria: body habitus with weight greater than } 150 \mathrm{~kg} \text { or estimated chest circumference } \\
\text { greater than } 150 \mathrm{~cm}, \text { pregnancy, time from emergency medical services call to physician arrive } \\
\text { greater than } 15 \mathrm{~min}\end{array}$ \\
\hline Interventions & $\begin{array}{l}\text { Mechanical group will receive chest compressions with AutoPulse device. Both the mechanical and } \\
\text { manual arms will follow the } 2005 \text { European Resuscitation Council Guidelines }\end{array}$
\end{tabular}

\section{Outcomes}

\section{Primary outcome}

- Rate of survival to admission to hospital with ROSC

\section{Secondary outcomes}

- Survival to 24-hours, to discharge from intensive care unit, to discharge from hospital, to three months, to one year

- Survival to each of these time points with good neurological function

\begin{tabular}{ll}
\hline Starting date & 10/01/2008 \\
\hline Contact information & Dr. Andreas Hoeft; email: andreas.hoeft@ukb.uni-bonn.de \\
\hline Notes & $\begin{array}{l}\text { Subgroup analysis will be performed for "fast AutoPulse", i.e. patients who receive mechanical } \\
\text { compressions in less than six minutes of the arrival of clinical staff }\end{array}$ \\
\hline
\end{tabular}

\section{NCT01521208}

\section{Trial name or title}

Methods

Participants
LUCAS continuous chest compressions in out-of-hospital cardiac arrest treatment: the LUCAT Trial

\author{
Randomised
}

Adults ( $>17$ years old and $<81$ years old) suffering from non-traumatic or unexpected witnessed (seen, heard or monitored) sudden cardiac arrest, attended by an advanced support ambulance (served by doctor or nurse) in Barcelona city, or in the Girona or Lleida area, with time between the emergency call and reaching patient less than 12 minutes.

Exclusion criteria include:

- biological signs of death

- younger than 18 years or older than 80 years 
NCT01521208 (Continued)

- trauma caused cardiorespiratory arrest (CRA), including hanging

- secondary CRA or intoxication

- return of spontaneous circulation previous to arrival of SEM medical team

- known pregnancy

- inadequate size for LUCAS device

- anything in the study that can delay treatment

\begin{tabular}{|c|c|}
\hline Interventions & Mechanical chest compressions performed by LUCAS device \\
\hline Outcomes & $\begin{array}{l}\text { Primary outcomes } \\
\text { - Survival at hospital admittance } \\
\text { - Survival on discharge from hospital } \\
\text { Secondary outcomes } \\
\text { - Restoration of spontaneous circulation } \\
\text { - End-tidal } \mathrm{CO}_{2} \text { values } \\
\text { - SOFA scale values } \\
\text { - Days before discharge from intensive care unit/coronary care unit } \\
\text { - Metabolic (pH, lactate) and inflammatory (leukocytes, C-reactive protein) parameters } \\
\text { - Epidemiology of out-of-hospital cardiac arrest } \\
\text { - Blood sample for genetic and biological studies } \\
\text { - Left ventricular function }\end{array}$ \\
\hline Starting date & January 2012 \\
\hline Contact information & $\begin{array}{l}\text { Dr. Francesc Carmona Jiménez; phone +34 } 6078477 \text { 17; email franciscojosecarmona@gencat.cat } \\
\text { Dr. Rosa-Maria Lidón; email rmlidon@vhebron.net }\end{array}$ \\
\hline Notes & NCT01521208 \\
\hline
\end{tabular}

\section{DATA AND ANALYSES}

\section{Comparison 1. Mechanical chest compressions versus manual chest compressions}

\begin{tabular}{lllll}
\hline Outcome or subgroup title & $\begin{array}{l}\text { No. of } \\
\text { studies }\end{array}$ & $\begin{array}{l}\text { No. of } \\
\text { partici- } \\
\text { pants }\end{array}$ & Statistical method & Effect size \\
\hline $\begin{array}{l}1 \text { Survival to hospital dis- } \\
\text { charge with good neurologi- } \\
\text { cal function }\end{array}$ & 3 & Risk Ratio $(\mathrm{M}-\mathrm{H}$, Random, $95 \% \mathrm{Cl})$ & Totals not selected \\
\hline $\begin{array}{l}2 \text { Survival to hospital dis- } \\
\text { charge }\end{array}$ & 7 & Risk Ratio $(\mathrm{M}-\mathrm{H}$, Random, $95 \% \mathrm{Cl})$ & Totals not selected \\
\hline $\begin{array}{l}3 \text { Return of spontaneous cir- } \\
\text { culation }\end{array}$ & 8 & Risk Ratio $(\mathrm{M}-\mathrm{H}$, Random, $95 \% \mathrm{Cl})$ & Totals not selected \\
\hline
\end{tabular}




\begin{tabular}{|c|c|c|c|c|}
\hline Outcome or subgroup title & $\begin{array}{l}\text { No. of } \\
\text { studies }\end{array}$ & $\begin{array}{l}\text { No. of } \\
\text { partici- } \\
\text { pants }\end{array}$ & Statistical method & Effect size \\
\hline $\begin{array}{l}4 \text { Survival to hospital admis- } \\
\text { sion }\end{array}$ & 4 & & Risk Ratio (M-H, Random, 95\% Cl) & Totals not selected \\
\hline 5 Sternal or rib fractures & 7 & & Risk Ratio (M-H, Random, 95\% Cl) & Totals not selected \\
\hline $\begin{array}{l}6 \text { Haemothorax or pneu- } \\
\text { mothorax }\end{array}$ & 5 & & Risk Ratio (M-H, Random, 95\% Cl) & Totals not selected \\
\hline $\begin{array}{l}7 \text { Internal abdominal organ } \\
\text { injury }\end{array}$ & 5 & & Risk Ratio (M-H, Random, 95\% Cl) & Totals not selected \\
\hline
\end{tabular}

Analysis 1.1. Comparison 1 Mechanical chest compressions versus manual chest compressions, Outcome 1 Survival to hospital discharge with good neurological function.

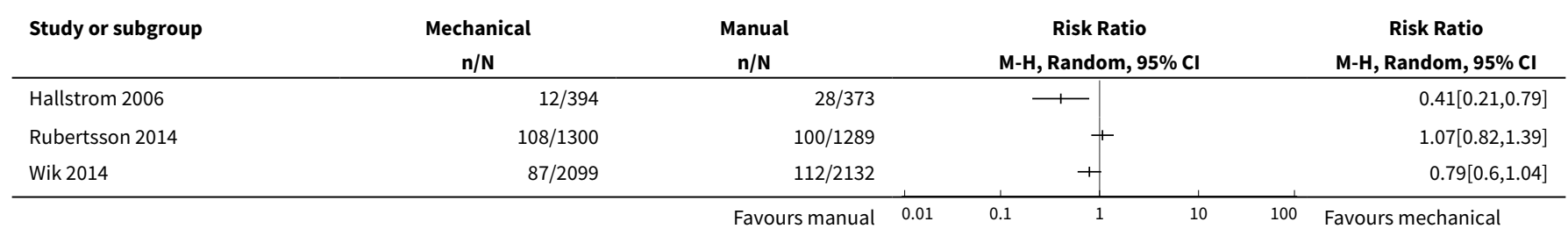

Analysis 1.2. Comparison 1 Mechanical chest compressions versus manual chest compressions, Outcome 2 Survival to hospital discharge.

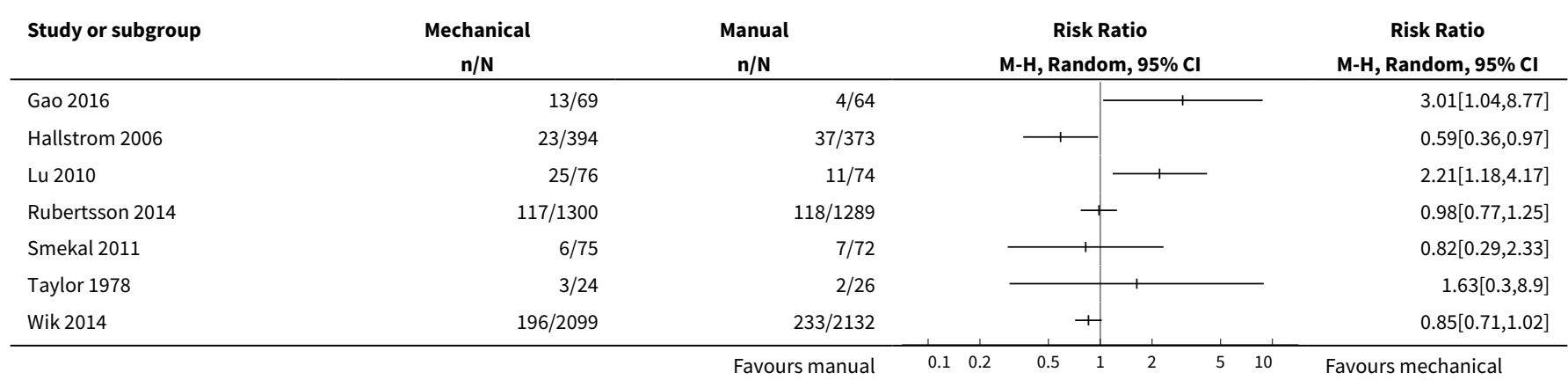

Analysis 1.3. Comparison 1 Mechanical chest compressions versus manual chest compressions, Outcome 3 Return of spontaneous circulation.

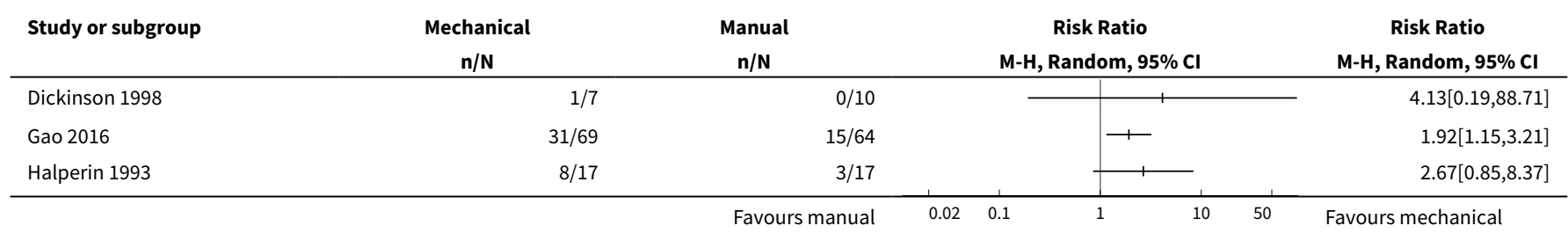




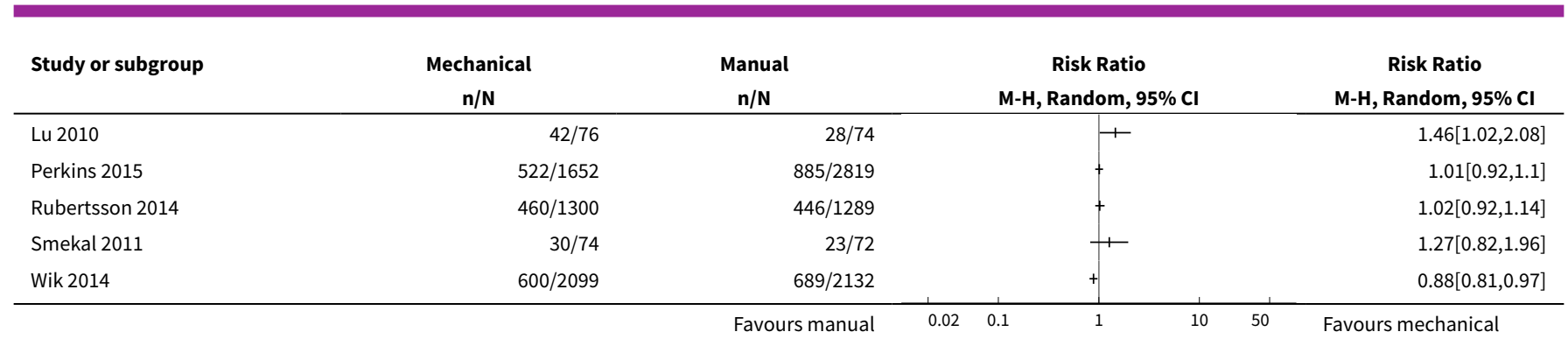

Analysis 1.4. Comparison 1 Mechanical chest compressions versus manual chest compressions, Outcome 4 Survival to hospital admission.

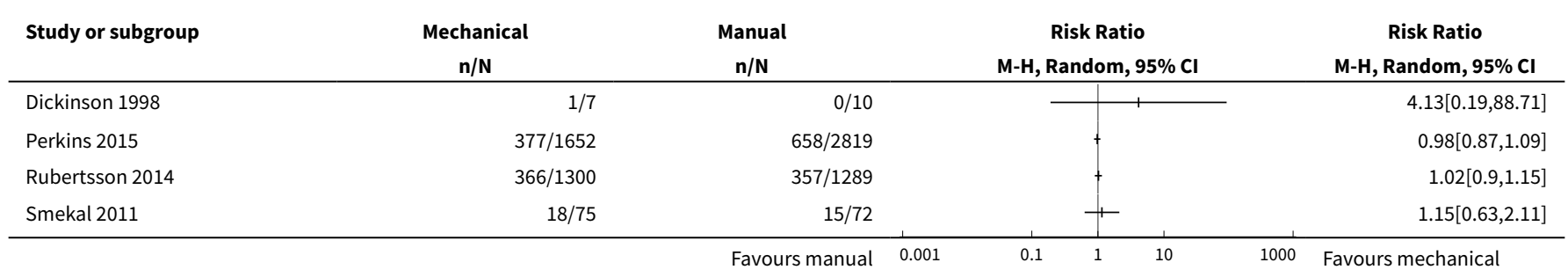

Analysis 1.5. Comparison 1 Mechanical chest compressions versus manual chest compressions, Outcome 5 Sternal or rib fractures.

\begin{tabular}{|c|c|c|c|c|}
\hline Study or subgroup & $\begin{array}{c}\text { Mechanical } \\
\mathbf{n} / \mathbf{N}\end{array}$ & $\begin{array}{c}\text { Manual } \\
\mathbf{n} / \mathbf{N}\end{array}$ & $\begin{array}{c}\text { Risk Ratio } \\
\text { M-H, Random, } 95 \% \text { Cl }\end{array}$ & $\begin{array}{c}\text { Risk Ratio } \\
\text { M-H, Random, 95\% Cl }\end{array}$ \\
\hline Gao 2016 & $4 / 60$ & $3 / 63$ & \begin{tabular}{l|l} 
\\
\end{tabular} & $1.4[0.33,6]$ \\
\hline Halperin 1993 & $1 / 4$ & $2 / 5$ & & $0.63[0.08,4.66]$ \\
\hline Koster 2017 & $90 / 211$ & $52 / 126$ & + & $1.03[0.8,1.34]$ \\
\hline Lu 2010 & $2 / 76$ & $8 / 74$ & & $0.24[0.05,1.11]$ \\
\hline Rubertsson 2014 & $1 / 1300$ & $2 / 1289$ & & $0.5[0.05,5.46]$ \\
\hline Taylor 1978 & $10 / 13$ & $8 / 17$ & 1 & $1.63[0.91,2.94]$ \\
\hline Wik 2014 & $70 / 2099$ & $36 / 2132$ & + & $1.98[1.33,2.94]$ \\
\hline
\end{tabular}

Analysis 1.6. Comparison 1 Mechanical chest compressions versus manual chest compressions, Outcome 6 Haemothorax or pneumothorax.

\begin{tabular}{|c|c|c|c|c|c|}
\hline Study or subgroup & $\begin{array}{c}\text { Mechanical } \\
\mathrm{n} / \mathrm{N}\end{array}$ & $\begin{array}{c}\text { Manual } \\
\mathbf{n} / \mathbf{N}\end{array}$ & & $\begin{array}{c}\text { Risk Ratio } \\
\text { M-H, Random, } 95 \% \mathrm{Cl}\end{array}$ & $\begin{array}{c}\text { Risk Ratio } \\
\text { M-H, Random, } 95 \% \mathrm{Cl}\end{array}$ \\
\hline Halperin 1993 & $0 / 4$ & $1 / 5$ & & 1 & $0.4[0.02,7.82]$ \\
\hline Koster 2017 & $14 / 211$ & $7 / 126$ & & + & $1.19[0.5,2.88]$ \\
\hline Lu 2010 & $1 / 76$ & $3 / 74$ & & & $0.32[0.03,3.05]$ \\
\hline Rubertsson 2014 & $1 / 1300$ & $1 / 1289$ & & & $0.99[0.06,15.84]$ \\
\hline Wik 2014 & $34 / 2099$ & $21 / 2132$ & & - & $1.64[0.96,2.82]$ \\
\hline
\end{tabular}


Analysis 1.7. Comparison 1 Mechanical chest compressions versus manual chest compressions, Outcome 7 Internal abdominal organ injury.

\begin{tabular}{|c|c|c|c|c|}
\hline Study or subgroup & $\begin{array}{c}\text { Mechanical } \\
\mathrm{n} / \mathrm{N}\end{array}$ & $\begin{array}{c}\text { Manual } \\
\mathbf{n} / \mathbf{N}\end{array}$ & $\begin{array}{c}\text { Risk Ratio } \\
\text { M-H, Random, 95\% Cl }\end{array}$ & $\begin{array}{c}\text { Risk Ratio } \\
\text { M-H, Random, } 95 \% \mathrm{CI}\end{array}$ \\
\hline Koster 2017 & $3 / 211$ & $0 / 126$ & & $4.19[0.22,80.52]$ \\
\hline Lu 2010 & $1 / 76$ & $2 / 74$ & & $0.49[0.05,5.26]$ \\
\hline Rubertsson 2014 & $1 / 1300$ & $0 / 1289$ & & $2.97[0.12,72.95]$ \\
\hline Taylor 1978 & $0 / 13$ & $2 / 17$ & & $0.26[0.01,4.94]$ \\
\hline Wik 2014 & $1 / 2099$ & $0 / 2132$ & & $3.05[0.12,74.76]$ \\
\hline
\end{tabular}

\section{APPENDICES}

\section{Appendix 1. Search strategies 2017 update}

The searches below were performed on 30 April 2015 and repeated on 19 August 2017 to capture records between those two dates.

\section{CENTRAL}

\#1 MeSH descriptor: [Heart Arrest] explode all trees

\#2 cardiac next arrest

\#3 heart next arrest

\#4 cardiopulmonary next arrest

\#5 sudden near/3 death

\#6 MeSH descriptor: [Death, Sudden] explode all trees

$\# 7$ (\#1 or \#2 or \#3 or \#4 or \#5 or \#6)

\#8 MeSH descriptor: [Cardiopulmonary Resuscitation] this term only

\#9 MeSH descriptor: [Heart Massage] this term only

$\# 10 \mathrm{cpr}$

$\# 11$ resuscitat ${ }^{\star}$

\#12 heart next massage

\#13 cardiac next massage

\#14 chest next compression

\#15 (\#8 or \#9 or \#10 or \#11 or \#12 or \#13 or \#14)

\#16 (\#7 and \#15)

\#17 piston

\#18 autopulse

\#19 auto-pulse

\#20 thumper

\#21 pneumatic 
\#22 lucas

\#23 hands-free

\#24 load next distributing

\#25 vest

\#26 mechanical near/6 compression

\#27 mechanical near/6 cpr

\#28 automat* near/6 compression

\#29 automat* near/6 cpr

\#30 device near/6 compression

\#31 device near/6 cpr

\#32 machine near/6 compression

\#33 machine near/6 cpr

\#34 MeSH descriptor: [Cardiopulmonary Resuscitation] this term only and with qualifiers: [Instrumentation - IS]

$\# 35$ (\#17 or \#18 or \#19 or \#20 or \#21 or \#22 or \#23 or \#24 or \#25)

$\# 36$ (\#26 or \#27 or \#28 or \#29 or \#30 or \#31 or \#32 or \#33 or \#34)

\#37 (\#35 or \#36)

\#38 (\#37 and \#16)

\section{MEDLINE Ovid}

1. exp Heart Arrest/

2. $\exp$ Death, Sudden/

3. cardiac arrest.tw.

4. heart arrest.tw.

5. cardiopulmonary arrest.tw.

6. sudden cardiac death\$.tw.

7. sudden death\$.tw.

8. or/1-7

9. exp Cardiopulmonary Resuscitation/

10. Heart Massage/

11. cpr.tw.

12. cardiopulmonary resuscitation.tw.

13. chest compression\$.tw.

14. resuscitat\$.tw.

15. or/9-14

16. Cardiopulmonary Resuscitation/is [Instrumentation]

17. autopulse.tw. 
18. auto-pulse.tw.

19. thumper.tw.

20. lucas.tw.

21. hands-free.tw.

22. (pneumatic adj10 (pump or device)).tw.

23. (pneumatic adj10 compression\$).tw.

24. (automat\$ adj10 compression\$).tw.

25. (device\$ adj10 compression).tw.

26. (mechanical adj10 compression\$).tw.

27. (machine\$ adj10 compression\$).tw.

28. piston\$.tw.

29. load distributing.tw.

30. (vest adj10 compression).tw.

31. (mechanical adj10 cpr).tw.

32. (pneumatic adj10 cpr).tw.

33. (device adj10 cpr).tw.

34. (machine $\$$ adj10 cpr).tw.

35. (vest adj10 cpr).tw.

36. or/16-35

37.8 and 15 and 36

38. randomised controlled trial.pt.

39. controlled clinical trial.pt.

40. randomized.ab.

41. placebo.ab.

42. drug therapy.fs.

43. randomly.ab.

44. trial.ab.

45. groups.ab.

46.38 or 39 or 40 or 41 or 42 or 43 or 44 or 45

47. exp animals/ not humans.sh.

48. 46 not 47

49. 37 and 48

50. $\left(2013^{\star}\right.$ or $2014^{\star}$ or $\left.2015^{\star}\right)$.ed. [ ${ }^{\star \star \star}$ The repeated search in 2017 featured only the years $2015-2017$ ]

51.49 and 50 


\section{Embase Ovid}

1. heart arrest/

2. sudden death/

3. cardiac arrest.tw.

4. heart arrest.tw.

5. cardiopulmonary arrest.tw.

6. sudden cardiac death\$.tw.

7. sudden death\$.tw.

8. or/1-7

9. resuscitation/

10. heart massage/

11. cpr.tw.

12. cardiopulmonary resuscitation.tw.

13. chest compression\$.tw.

14. resuscitat\$.tw.

15. or/9-14

16. cardiovascular equipment/

17. autopulse.tw.

18. auto-pulse.tw.

19. thumper.tw.

20. lucas.tw.

21. hands-free.tw.

22. (pneumatic adj10 (pump or device)).tw.

23. (pneumatic adj10 compression\$).tw.

24. (automat\$ adj10 compression\$).tw.

25. (device\$ adj10 compression).tw.

26. (mechanical adj10 compression\$).tw.

27. (machine\$ adj10 compression\$).tw.

28. piston\$.tw.

29. load distributing.tw.

30. (vest adj10 compression).tw.

31. (mechanical adj10 cpr).tw.

32. (pneumatic adj10 cpr).tw.

33. (device adj10 cpr).tw.

34. (machine $\$$ adj10 cpr).tw. 
35. (vest adj10 cpr).tw.

36. or/16-35

37.8 and 15 and 36

38. random\$.tw.

39. factorial\$.tw.

40. crossover\$.tw.

41. cross over\$.tw.

42. cross-over\$.tw.

43. placebo\$.tw.

44. (doubl\$ adj blind\$).tw.

45. (singl\$ adj blind\$).tw.

46. assign\$.tw.

47. allocat\$.tw.

48. volunteer\$.tw.

49. crossover procedure/

50. double blind procedure/

51. randomised controlled trial/

52. single blind procedure/

53. 38 or 39 or 40 or 41 or 42 or 43 or 44 or 45 or 46 or 47 or 48 or 49 or 50 or 51 or 52

54. (animal/ or nonhuman/) not human/

55.53 not 54

56.37 and 55

57. $\left(2013^{\star}\right.$ or $2014^{\star}$ or $\left.2015^{\star}\right)$.em [ ${ }^{\star \star \star}$ The repeated search in 2017 featured only the years $2015-2017$ ]

58.56 and 57

\section{Web of Science}

\# 7 \#6 Databases=SCI-EXPANDED, CPCI-S Timespan=2013 to 28-4-2015 [ ${ }^{\star \star \star}$ The repeated search in 2017 featured only the years 2015-2017]

\# 6 \#5 AND \#4

\# 5 TS=(random ${ }^{\star}$ or blind ${ }^{\star}$ or allocat ${ }^{\star}$ or assign* or trial $^{\star}$ or placebo* or crossover $^{\star}$ or cross-over $\left.^{\star}\right)$

\# 4 \#3 AND \#2 AND \#1

\# $3 \mathrm{TS}=\left(\right.$ mechanical $^{\star}$ or device ${ }^{\star}$ or automat $\left.^{\star}\right)$

\# 2 TS=(cardiopulmonary resuscitation or cpr or chest compression*)

\# 1 TS=(cardiac arrest or sudden death or cardiopulmonary arrest)

\section{ClinicalTrials.gov}

1. Cardiac arrest and device*

2. Cardiac arrest and mechanical

3. 1 or 2 


\section{WHO International Clinical Trials Registry Platform}

1. Cardiac arrest and mechanical

2. lucas

3. autopulse

4. 1 or 2 or 3

\section{Appendix 2. Search strategies 2014 update}

\section{CENTRAL}

\#1 MeSH descriptor: [Heart Arrest] explode all trees

\#2 cardiac next arrest

\#3 heart next arrest

\#4 cardiopulmonary next arrest

\#5 sudden near/3 death

\#6 MeSH descriptor: [Death, Sudden] explode all trees

$\# 7$ (\#1 or \#2 or \#3 or \#4 or \#5 or \#6)

\#8 MeSH descriptor: [Cardiopulmonary Resuscitation] this term only

\#9 MeSH descriptor: [Heart Massage] this term only

$\# 10 \mathrm{cpr}$

$\# 11$ resuscitat ${ }^{\star}$

\#12 heart next massage

$\# 13$ cardiac next massage

\#14 chest next compression

\#15 (\#8 or \#9 or \#10 or \#11 or \#12 or \#13 or \#14)

$\# 16$ (\#7 and \#15)

\#17 piston

\#18 autopulse

\#19 auto-pulse

\#20 thumper

\#21 pneumatic

\#22 lucas

\#23 hands-free

\#24 load next distributing

\#25 vest

\#26 mechanical near/6 compression

\#27 mechanical near/6 cpr

\#28 automat* near/6 compression

\#29 automat* near/6 cpr

\#30 device near/6 compression

\#31 device near/6 cpr

\#32 machine near/6 compression

\#33 machine near/6 cpr

\#34 MeSH descriptor: [Cardiopulmonary Resuscitation] this term only and with qualifiers: [Instrumentation - IS]

$\# 35$ (\#17 or \#18 or \#19 or \#20 or \#21 or \#22 or \#23 or \#24 or \#25)

$\# 36$ (\#26 or \#27 or \#28 or \#29 or \#30 or \#31 or \#32 or \#33 or \#34)

\#37 (\#35 or \#36)

\#38 (\#37 and \#16)

\section{MEDLINE Ovid}

1. exp Heart Arrest/

2. exp Death, Sudden/

3. cardiac arrest.tw.

4. heart arrest.tw.

5. cardiopulmonary arrest.tw.

6. sudden cardiac death\$.tw.

7. sudden death\$.tw.

8. or/1-7

9. exp Cardiopulmonary Resuscitation/

10. Heart Massage/

11. cpr.tw.

12. cardiopulmonary resuscitation.tw. 
13. chest compression\$.tw.

14. resuscitat\$.tw.

15. or/9-14

16. Cardiopulmonary Resuscitation/is [Instrumentation]

17. autopulse.tw.

18. auto-pulse.tw.

19. thumper.tw.

20. lucas.tw.

21. hands-free.tw.

22. (pneumatic adj10 (pump or device)).tw.

23. (pneumatic adj10 compression\$).tw.

24. (automat\$ adj10 compression\$).tw.

25. (device $\$$ adj10 compression).tw.

26. (mechanical adj10 compression\$).tw.

27. (machine $\$$ adj10 compression\$).tw.

28. piston\$.tw.

29. load distributing.tw.

30. (vest adj10 compression).tw.

31. (mechanical adj10 cpr).tw.

32. (pneumatic adj10 cpr).tw.

33. (device adj10 cpr).tw.

34. (machine $\$$ adj10 cpr).tw.

35. (vest adj10 cpr).tw.

36. or $/ 16-35$

37.8 and 15 and 36

38. randomised controlled trial.pt.

39. controlled clinical trial.pt.

40. randomized.ab.

41. placebo.ab.

42. drug therapy.fs.

43. randomly.ab.

44. trial.ab.

45. groups.ab.

46. 38 or 39 or 40 or 41 or 42 or 43 or 44 or 45

47. exp animals/ not humans.sh.

48. 46 not 47

49.37 and 48

50. $\left(200911^{\star}\right.$ or $2010^{\star}$ or $2011^{\star}$ or $2012^{\star}$ or $\left.2013^{\star}\right)$.ed.

51.49 and 50

\section{Embase Ovid}

1. heart arrest/

2. sudden death/

3. cardiac arrest.tw.

4. heart arrest.tw.

5. cardiopulmonary arrest.tw.

6. sudden cardiac death\$.tw.

7. sudden death\$.tw.

8. or/1-7

9. resuscitation/

10. heart massage/

11. cpr.tw.

12. cardiopulmonary resuscitation.tw.

13. chest compression\$.tw.

14. resuscitat\$.tw.

15. or $/ 9-14$

16. cardiovascular equipment/

17. autopulse.tw.

18. auto-pulse.tw.

19. thumper.tw.

20. lucas.tw. 
21. hands-free.tw.

22. (pneumatic adj10 (pump or device)).tw.

23. (pneumatic adj10 compression\$).tw.

24. (automat\$ adj10 compression\$).tw.

25. (device $\$$ adj10 compression).tw.

26. (mechanical adj10 compression\$).tw.

27. (machine $\$$ adj10 compression\$).tw.

28. piston\$.tw.

29. load distributing.tw.

30. (vest adj10 compression).tw.

31. (mechanical adj10 cpr).tw.

32. (pneumatic adj10 cpr).tw.

33. (device adj10 cpr).tw.

34. (machine $\$$ adj10 cpr).tw.

35. (vest adj10 cpr).tw.

36. or/16-35

37.8 and 15 and 36

38. random $\$$.tw.

39. factorial\$.tw.

40. crossover\$.tw.

41. cross over\$.tw.

42. cross-over\$.tw.

43. placebo\$.tw.

44. (doubl\$ adj blind\$).tw.

45. (singl\$ adj blind\$).tw.

46. assign\$.tw.

47. allocat\$.tw.

48. volunteer\$.tw.

49. crossover procedure/

50. double blind procedure/

51. randomised controlled trial/

52. single blind procedure/

53.38 or 39 or 40 or 41 or 42 or 43 or 44 or 45 or 46 or 47 or 48 or 49 or 50 or 51 or 52

54. (animal/ or nonhuman/) not human/

55.53 not 54

56.37 and 55

57. ("200945" or "200946" or "200947" or "200948" or "200949" or $20095^{\star}$ or $2010^{\star}$ or $2011^{\star}$ or $2012^{\star}$ or $\left.2013^{\star}\right)$.em.

58.56 and 57

\section{Web of Science}

\# 7 \#6 Databases=SCI-EXPANDED, CPCI-S Timespan=2009-11-18 - 2013-01-23

\# 6 \#5 AND \#4

\# 5 TS $=\left(\right.$ random $^{\star}$ or blind ${ }^{\star}$ or allocat ${ }^{\star}$ or assign ${ }^{\star}$ or trial ${ }^{\star}$ or placebo* or crossover $^{\star}$ or cross-over $\left.^{\star}\right)$

\# 4 \#3 AND \#2 AND \#1

\# 3 TS $=\left(\right.$ mechanical $^{\star}$ or device ${ }^{\star}$ or automat $\left.^{\star}\right)$

\# 2 TS=(cardiopulmonary resuscitation or cpr or chest compression $\left.{ }^{\star}\right)$

\# 1 TS=(cardiac arrest or sudden death or cardiopulmonary arrest)

\section{ClinicalTrials.gov}

1. Cardiac arrest and device*

2. Cardiac arrest and mechanical

3. 1 or 2

\section{Appendix 3. Search strategies for original review published in 2011}

\section{CENTRAL on the Cochrane Library}

\#1 MeSH descriptor heart arrest explode all trees

\#2 cardiac next arrest in All Text

\#3 heart next arrest in All Text

\#4 cardiopulmonary next arrest in All Text

\#5 sudden near/3 death in All Text 
\#6 MeSH descriptor Death, Sudden explode all trees

\#7 (\#1 or \#2 or \#3 or \#4 or \#5 or \#6)

\#8 MeSH descriptor Cardiopulmonary Resuscitation this term only

\#9 MeSH descriptor heart massage this term only

$\# 10 \mathrm{cpr}$ in All Text

\#11 resuscitat* in All Text

\#12 heart next massage in All Text

\#13 cardiac next massage in All Text

\#14 chest next compression in All Text

$\# 15$ (\#8 or \#9 or \#10 or \#11 or \#12 or \#13 or \#14)

\#16 (\#7 and \#15) 5

$\# 17$ piston in All Text

$\# 18$ autopulse in All Text

$\# 19$ auto-pulse in All Text

\#20 thumper in All Text

\#21 pneumatic in All Text

\#22 lucas in All Text

\#23 hands-free in All Text

\#24 load next distributing in All Text

\#25 vest in All Text

\#26 (mechanical in All Text near/6 compression in All Text)

\#27 (mechanical in All Text near/6 cpr in All Text)

\#28 (automat ${ }^{\star}$ in All Text near/6 compression in All Text)

\#29 (automat* in All Text near/6 cpr in All Text)

\#30 (device in All Text near/6 compression in All Text)

\#31 (device in All Text near/6 cpr in All Text)

\#32 (machine in All Text near/6 compression in All Text)

\#33 (machine in All Text near/6 cpr in All Text)

\#34 MeSH descriptor cardiopulmonary resuscitation this term only with qualifiers: IS

$\# 35$ (\#17 or \#18 or \#19 or \#20 or \#21 or \#22 or \#23 or \#24 or \#25)

$\# 36$ (\#26 or \#27 or \#28 or \#29 or \#30 or \#31 or \#32 or \#33 or \#34)

\#37 (\#35 or \#36)

\#38 (\#37 and \#16)

\section{MEDLINE OVID}

1. exp Heart Arrest/

2. exp Death, Sudden/

3. cardiac arrest.tw.

4. heart arrest.tw.

5. cardiopulmonary arrest.tw.

6. sudden cardiac death $\$ . t w$.

7. sudden death\$.tw.

8. or/1-7

9. exp Cardiopulmonary Resuscitation/

10. Heart Massage/

11. cpr.tw.

12. cardiopulmonary resuscitation.tw.

13. chest compression\$.tw.

14. resuscitat\$.tw.

15. or/9-14 (33098)

16. Cardiopulmonary Resuscitation/is [Instrumentation]

17. autopulse.tw.

18. auto-pulse.tw.

19. thumper.tw.

20. lucas.tw.

21. hands-free.tw.

22. (pneumatic adj10 (pump or device)).tw.

23. (pneumatic adj10 compression\$).tw.

24. (automat\$ adj10 compression\$).tw.

25. (device $\$$ adj10 compression).tw.

26. (mechanical adj10 compression\$).tw. 
27. (machine\$ adj10 compression\$).tw.

28. piston\$.tw.

29. load distributing.tw.

30. (vest adj10 compression).tw.

31. (mechanical adj10 cpr).tw.

32. (pneumatic adj10 cpr).tw.

33. (device adj10 cpr).tw.

34. (machine adj10 cpr).tw.

35. (vest adj10 cpr).tw.

36. or/16-35

37.8 and 15 and 36

38. randomised controlled trial.pt.

39. controlled clinical trial.pt.

40. randomised controlled trials.sh.

41. random allocation.sh.

42. double blind method.sh.

43. single blind method.sh.

44. or/38-43

45. (animals not humans).sh.

46. 44 not 45

47. clinical trial.pt.

48. exp clinical trials/

49. (clin\$ adj25 trial\$).ti,ab.

50. ((singl\$ or doubl\$ or trebl\$ or tripl\$) adj25 (blind\$ or mask\$)).ti,ab.

51. placebos.sh.

52. placebo\$.ti,ab.

53. random $\$ . t i, a b$.

54. research design.sh.

55. or/47-54

56.55 not 45

57.56 not 46

58. comparative study.sh.

59. exp evaluation studies/

60. follow up studies.sh.

61. prospective studies.sh.

62. (control\$ or prospectiv\$ or volunteer\$).ti,ab.

63. or $/ 58-62$

64.63 not 45

65.64 not ( 46 or 57$)$

66.46 or 57 or 65

67.37 and 66

\section{Embase OVID}

1. Heart Arrest/

2. Sudden Death/

3. cardiac arrest.tw.

4. heart arrest.tw.

5. cardiopulmonary arrest.tw.

6. sudden cardiac death\$.tw.

7. sudden death\$.tw.

8. or/1-7

9. Resuscitation/

10. Heart Massage/

11. cpr.tw.

12. cardiopulmonary resuscitation.tw.

13. chest compression\$.tw.

14. resuscitat\$.tw.

15. or/9-14

16. Cardiovascular Equipment/

17. autopulse.tw.

18. auto-pulse.tw. 
19. thumper.tw.

20. lucas.tw.

21. hands-free.tw.

22. (pneumatic adj10 (pump or device)).tw.

23. (pneumatic adj10 compression\$).tw.

24. (automat\$ adj10 compression\$).tw.

25. (device $\$$ adj10 compression).tw.

26. (mechanical adj10 compression\$).tw.

27. (machine $\$$ adj10 compression\$).tw.

28. piston\$.tw.

29. load distributing.tw.

30.(vest adj10 compression).tw.

31. (mechanical adj10 cpr).tw.

32. (pneumatic adj10 cpr).tw.

33. (device adj10 cpr).tw.

34. (machine $\$$ adj10 cpr).tw.

35. (vest adj10 cpr).tw.

36. or/16-35

37.8 and 15 and 36

38. clinical trial/

39. random\$.tw.

40. randomised controlled trial/

41. trial\$.tw.

42. follow-up.tw.

43. double blind procedure/

44. placebo\$.tw.

45. placebo/

46. factorial\$.ti,ab.

47. (crossover\$ or cross-over\$).ti,ab.

48. (double\$ adj blind\$).ti,ab.

49. (singl\$ adj blind\$).ti,ab.

50. assign \$.ti,ab.

51. allocat\$.ti,ab.

52. volunteer $\$$.ti, ab.

53. Crossover Procedure/

54. Single Blind Procedure/

55. or/38-54

56. (exp animal experiment/ or nonhuman/) not exp human/

57.55 not 56

58. 37 and 57

\section{Science Citations Index and Biotech and Bioengineering abstracts}

cardiac arrest or sudden death or cardiopulmonary arrest

AND

cardiopulmonary resuscitation or cpr or chest compression*

AND

mechanical $^{\star}$ or device ${ }^{\star}$ or automat*

\section{ClinicalTrials.gov}

1. Cardiac arrest and device*

2. Cardiac arrest and mechanical

3. 1 or 2

\section{Appendix 4. Glossary}

\section{The CPC (Cerebral Performance Category) score}

The Cerebral Performance Category scale is a simple five-point measurement of cerebral and functional status that ranges from category one to indicate good performance to category five to indicate brain death. The Cerebral Performance Category scale is widely used to evaluate functional outcome in resuscitation research because it requires little training, is brief and can be evaluated with the health record.

\section{The modified Rankin Scale (mRS)}


The modified Rankin scale is a single-item, 6-point measurement of primarily functional domains that range from zero (no impairment) to six (deceased). This is usually used on patients who have suffered a stroke or other neurological insult to measure functional dependency postinjury. The mRS may be determined through chart review.

\section{Utstein guidelines}

The Utstein style for reporting cardiac arrests arose from a 1990 conference at the ancient abbey of that name on an island near Stavanger, Norway. That conference and another later that year were attended by representatives of the AHA, the European Resuscitation Council, the Heart and Stroke Foundation of Canada and the Australian Resuscitation Council. The major concern was that the results of resuscitation endeavours in different countries, and even within countries, could not be compared meaningfully. Researchers have used disparate endpoints to assess the effectiveness of different systems and interventions. Useful comparisons have been prevented by this lack of uniform definitions and standard methodologies. The Utstein guidelines are a defined set of data elements that are essential or desirable for documenting in-hospital cardiac arrest. Data categories are hospital variables, patient variables, arrest variables and outcome variables. The 'In-Hospital Utstein-Style Template' was developed to summarise these data and recommendations for reporting a specific set of survival rates and outcomes. The Utstein style has attracted wide interest and has become a familiar term among members of the resuscitation community. Many researchers and system directors have adopted the Utstein templates, style and nomenclature to report results of prehospital resuscitation. The success of this international initiative soon led to uniform international styles for reporting the results of paediatric resuscitation and experimental (laboratory) resuscitation.

\section{WHAT'S NEW}

\begin{tabular}{lll}
\hline Date & Event & Description \\
\hline 31 August 2017 & $\begin{array}{l}\text { New citation required but conclusions } \\
\text { have not changed }\end{array}$ & $\begin{array}{l}\text { We identified five new studies for this update (Gao 2016; Koster } \\
\text { 2017; Perkins 2015; Rubertsson 2014; Wik 2014). }\end{array}$ \\
\hline 31 August 2017 & New search has been performed & We updated the searches to August 2017. \\
\hline
\end{tabular}

\section{H I S T O R Y}

Protocol first published: Issue 3, 2008

Review first published: Issue 1, 2011

\begin{tabular}{lll}
\hline Date & Event & Description \\
\hline 13 January 2014 & $\begin{array}{l}\text { New citation required but conclusions } \\
\text { have not changed }\end{array}$ & $\begin{array}{l}\text { Two new citations have been identified in this update (Smekal } \\
2011 \text { and Lu 2010), but the data contained within have not } \\
\text { changed our conclusion. }\end{array}$ \\
\hline 7 August 2013 & New search has been performed & $\begin{array}{l}\text { Review updated with new search dated January 2013. New au- } \\
\text { thor Nizar Hassan added. }\end{array}$ \\
\hline
\end{tabular}

\section{CONTRIBUTIONS OF AUTHORS}

Steven Brooks: guarantor for the review, conception of the study question, protocol draft development and revision, search strategy development and execution, selection of studies, quality review, data abstraction, data analysis, supervised Peter Wang in the process of conducting the review as first author.

Peter Wang: contributed to the 2017 update: quality review, data abstraction, data analysis, manuscript update drafting, editing.

\section{DECLARATIONS OF INTEREST}

Steven Brooks has received salary support from the Heart and Stroke Foundation to study the implementation of public access defibrillation. Dr. Brooks receives funding from the Canadian Institutes of Health Research to conduct research on the PulsePoint mobile app for cardiac arrest. Dr. Brooks has received operating funds from the Heart and Stroke Foundation and the Canadian Institutes of Health Research for various other peer-reviewed research studies in cardiac arrest. Dr. Brooks is a paid expert witness for the defense in a variety 
of civil cases involving the non-use of a defibrillator in a case of out-of-hospital cardiac arrest, misdiagnosis of chest pain and misdiagnosis of meningitis.

Peter Wang has no conflicts to report.

\section{SOURCES OF SUPPORT}

\section{Internal sources}

- No sources of support supplied

\section{External sources}

- This project was supported by the National Institute for Health Research (NIHR), via Cochrane Infrastructure to the Heart Group. The views and opinions expressed therein are those of the authors and do not necessarily reflect those of the Systematic Reviews Programme, NIHR, National Health Service (NHS) or the Department of Health, UK.

\section{INDEX TERMS}

\section{Medical Subject Headings (MeSH)}

Blood Circulation; Cardiopulmonary Resuscitation [instrumentation] [methods] [mortality]; Heart Arrest [mortality] [therapy]; Heart Massage [instrumentation] [*methods] [mortality]; Hospitalization; Patient Discharge; Randomized Controlled Trials as Topic

\section{MeSH check words}

Humans 